\title{
2017 ASSBI 40th Annual Conference Abstracts
}

\author{
THURSDAY 1st JUNE
}

Workshop 1

\section{Developing and Evaluating Complex Interventions: Putting the Medical Research Council Framework into Practice}

Roshan das Nair

The University of Nottingham, Nottingham, UK

\begin{abstract}
T 2000 (and updated in 2008), the UK Medical Research Council (MRC) produced a framework for the development and evaluation of complex interventions. This was followed by guidance on process evaluation. These MRC Frameworks have been influential in healthcare research. At this workshop, using examples from our research on the development and evaluation of cognitive rehabilitation, we will discuss how we can use these frameworks in our own work, consider how we can ensure the fidelity of the delivery of our interventions, and how best to report our interventions for replication in clinical practice or other research studies.
\end{abstract}

\section{Workshop 2}

\section{Goal Setting, Motivation, and Patient Engagement in Neurorehabilitation}

William Levack

Rehabilitation Teaching Research Unit, University of Otago, Wellington, New Zealand

eurorehabilitation is a specialist area of healthcare that has patient en-
gagement at its core. The majority of neurorehabilitation interventions require patient involvement to be implemented, with the degree of this involvement heavily influencing the effectiveness of these interventions. This workshop will examine factors associated with patient engagement in neurorehabilitation, with a particular focus on the application of goal setting as a tool for enhancing a person's active involvement in therapy and rehabilitation planning. Consideration will be given to how people's needs change over the course of their recovery and how health professionals should alter their behaviour and methods of goal setting to address these needs at different stages of neurorehabilitation. The workshop will involve tutorials on motivation theory related to goal setting, discussion of contextual factors such as ethnicity and patient preferences for engagement, application of taught concepts to practice-based case studies and group-based activities to explore attitudes and experiences of goal setting in clinical practice. 
Workshop 3

\title{
What are We Doing when We Treat Our Patients? A Scheme-in-Progress for Analyzing the Active Ingredients of Rehabilitation
}

\author{
Tessa Hart \\ Moss Rehabilitation Research Institute, Elkins Park, PA, USA
}

$R$ ehabilitation is often said to be 'more than the sum of its parts'. But what, greatest difference to the outcomes sought by our patients; that is, what are the active ingredients of rehabilitation? In this workshop, we consider both the importance of this question and potential ways to begin to answer it, with an emphasis on rehabilitation treatments of most relevance to patients with acquired brain injury (ABI).

The majority of rehabilitation treatments do not exert their effects passively (as do 'medical' treatments such as medications and surgery). Rather, most of our interventions involve complex scenarios in which patient engagement, active learning and interaction with the provider and the environment are key components. Add to this the diversity of goals addressed in rehabilitation and the dynamics of a rehabilitation team, and we have a treatment package whose active ingredients are difficult to characterize, let alone to measure. It is little wonder that we are typically left with crude measures of treatment such as the number of days or hours spent, with little information as to what was actually done inside that 'black box'. Yet it is only by specifying and measuring the ingredients of rehabilitation that we can advance the scientific evidence base, with the ultimate hope of developing treatment guidelines for particular problems and particular kinds of patients. Arriving at a common language for specifying the ingredients of rehabilitation would also benefit therapist training and program evaluation efforts, as well as improving communication across the team, and between provider and patient/ family.

Previous attempts at characterizing rehabilitation treatments have been mostly 'bottom-up' or inductive, in which therapists list and quantify the specific activities included in their interventions. While useful for some purposes, these approaches have significant shortcomings. The bulk of this workshop will be devoted to discussing and illustrating an alternative scheme under development by a US-funded, multi-disciplinary group of rehabilitation researchers known as the Rehabilitation Treatment Taxonomy (RTT) group. Our scheme is 'top-down', in that it uses treatment theory as a basis for characterizing the interventions used in rehabilitation. We have elaborated a tripartite structure of treatment theory that is necessary and sufficient to explain any treatment's effects. This tripartite structure consists of a treatment target, which is a specific and measurable aspect of functioning selected for change; a set of active ingredients that, in some combination, are hypothesized to effect the desired changes in the target; and the mechanism(s) of action, which specifies the processes by which ingredients bring about these changes.

We have also identified three broad clusters of rehabilitation treatment targets that are mutually exclusive with respect to their essential active ingredients and hypothesized mechanisms of action: changes in organ functions, changes in skilled performances, and changes in cognitive and affective representations, i.e., factual knowledge, attitudes, and beliefs. The latter two are of particular importance to treatment of ABI, as by definition, the organ responsible for learning and for cognitive representation has been damaged. In this workshop, we will examine the basic rationale and terminology associated 
with the RTT scheme; explore the targets, ingredients, and mechanisms of action within each treatment cluster, with an emphasis on learning-based treatments; and discuss real-world examples of treatments for ABI in relation to the specification of their active ingredients. Participants will have opportunities to work with the RTT concepts using their own examples, to practice specifying treatments with respect to the targets within them and the ingredients that are hypothesized to be active, i.e., to effect changes, in each.

\title{
Workshop 4
}

\section{Clinical Presentations and Neurobiology of Younger Onset Dementia Syndromes}

Olivier Piguet

Brain and Mind Centre, the University of Sydney, Sydney, Australia

School of Psychology, the University of Sydney, Sydney, Australia

ARC Centre of Excellence in Cognition and its Disorders, Sydney, Australia

\begin{abstract}
$F$ rontotemporal dementia is a common cause of dementia before the age of - 65 years. Despite recent codification of its main clinical syndromes, detection and diagnosis remain suboptimal because of overlap in clinical features with other neurological (e.g., Alzheimer's disease) and psychiatric (e.g., major depressive disorders) conditions. In addition, the prediction of the underlying pathology in frontotemporal dementia syndromes in life is variable. This workshop will review the latest research relevant to the main syndromes of younger onset dementia, focusing on frontotemporal dementia, Alzheimer's disease, and the primary progressive aphasias. The following topics will be discussed: current diagnostic criteria, clinical and neuroimaging profiles, as well as neuropathology and genetic risks of these dementias. The workshop will provide attendees with tools to improve diagnosis of dementia syndromes, an understanding of their possible trajectories, and their impact on patient management and carer burden.
\end{abstract}

\section{FRIDAY 2nd JUNE}

Plenary 1

\section{Memory Rehabilitation in Acquired Brain Injuries: On the Bumpy Road to Establishing Evidence}

Roshan das Nair

The University of Nottingham, Nottingham, UK

\begin{abstract}
$M$ emory problems are a common complaint in acquired brain injuries (ABIs). Clinical guidelines on management of ABIs recommend that clinicians need to assess for and treat cognitive problems. Neuropsychological rehabilitation has been found to be effective in reducing certain cognitive problems in some neurological conditions. However, the research evidence for the effectiveness of such interventions for people with ABIs who have memory problems is equivocal. Where effectiveness has been established, this mainly tends to be on short-term impairment level measures that lack ecological validity, and not on long-term activity restrictions or participation (functional) outcomes.
\end{abstract}


In this presentation, I will review the current evidence of memory rehabilitation in ABIs, mainly focusing on multiple sclerosis, stroke, and traumatic brain injury, by sharing results of our systematic reviews, randomised controlled trials, and qualitative studies related to memory rehabilitation. I will discuss some of the key issues with current studies (and evaluation of complex interventions in general), and offer some thoughts about future research directions for evaluating the effectiveness of memory rehabilitation.

Correspondence: Roshan das Nair; roshan.dasnair@nottingham.ac.uk

\title{
Concurrent Session 1
}

\section{Using Google Calendar to Increase Independence in Adults with Severe Traumatic Brain Injury}

\author{
Susan Petrie, ${ }^{1}$ Natasha A. Lannin, ${ }^{2,3}$ Kate Phillips ${ }^{1}$ and Trish Johnson ${ }^{4}$ \\ ${ }^{1}$ Independent Rehabilitation Services, Ashburton, Victoria, Australia \\ ${ }^{2}$ Occupational Therapy Department, Alfred Health, Melbourne, Australia \\ ${ }^{3}$ Occupational Therapy, School of Allied Health, La Trobe University, Melbourne, Australia \\ ${ }^{4}$ Speech Pathology Australia, Melbourne, Australia
}

$\mathcal{R}$ ackground and aims: The evidence base for using compensatory strate3 gies to prompt prospective memory after traumatic brain injury (TBI) is well established and mainstream technology presents an opportunity to enhance memory recall. Emerging evidence from the UK suggests that Google Calendar (a free, mainstream electronic calendar) is able to overcome memory difficulties. We sought to test the feasibility of using Google Calendar to improve participation in activities of daily living in adults with TBI and to explore whether successful use was associated with a reduction in paid care hours.

Method: Before-and-after study design. $N=12$ participants were recruited from a community neurorehabilitation service. After a 2-week baseline period, participants received 8-weeks of Google Calendar training to address memory goals with an occupational therapist. Goals were identified and rated using the Canadian Occupational Performance Measure (COPM) and the Goal Attainment Scale (GAS). Participants underwent brief neuropsychological testing pre-intervention and completed the Rivermead Behavioural Memory Test 3 (RBMT 3) pre- and post-intervention.

Results: Mean age of participants was 32 years $(S D$ 9). Clinically important improvement in GAS $T$-Scores (mean change 19.8) and COPM performance $(p=.003)$ and satisfaction scores $(p=.005)$ were recorded. There were no significant changes in memory performance (mean change $1.75, n s$ ) or attendant care hours (6.5 mean reduction, $n s$ ).

Conclusions: This study provides preliminary evidence that Google Calendar is feasible to improve participation in adults with severe TBI when trained over an 8-week period. Sample size was underpowered and lack of a control group means a randomized control trial is recommended.

Correspondence: Susan Petrie; spetrie@ independent-rehab.com.au 


\title{
Successes and Challenges in Translating a Cognitive-Behavioural Memory Group Intervention into a Community-Based Organisation for People with Mild Cognitive Impairment
}

Glynda Kinsella, ${ }^{1,2}$ Elizabeth Mullaly, ${ }^{2}$ Kerryn Pike, ${ }^{1}$ Elizabeth Rand, ${ }^{2}$ Ann Reilly ${ }^{3}$ and Marina Cavuoto ${ }^{1,2}$

\author{
${ }^{1}$ School of Psychology \& Public Health, La Trobe University, Melbourne, Australia \\ ${ }^{2}$ Caulfield Hospital, Melbourne, Australia \\ ${ }^{3}$ Alzheimer's Australia Vic, Melbourne, Australia
}

\begin{abstract}
$\boldsymbol{R}$ ackground and aims: Our group has demonstrated that older people can 3 benefit from training to improve memory. This is important for people with mild cognitive impairment (MCI), a risk factor for development of dementia, for which current community services are limited. This study evaluates the feasibility of translating an RCT-evaluated memory group program (the La Trobe-Caulfield Hospital [LaTCH] Memory Group) into the community support services of Alzheimer's Australia Vic.

Method: Over 3 years, staff at Alzheimer's Australia VIC were trained as facilitators of the LaTCH Memory Group for people with MCI. Seven staff members were interviewed regarding their perceptions of gains by clients in LaTCH groups, change in their own practice after running the groups, and factors that assisted or formed barriers in implementing the program.

Results: Using qualitative analysis ('Most Significant Change' technique), staff commonly reported client improvement in self-confidence in managing memory, which reduced anxiety and increased re-engagement in life activities. A common theme of positive change in the staff's own practice related to increased practical knowledge of memory changes with age and health, leading to greater role satisfaction. Staff also spoke about several challenges in running and sustaining the program, for example, constraints in delivering a tightly manualised program.

Conclusions: Memory interventions delivered in a community setting can be effective and increase opportunities for older people with memory problems to access services. Evaluation of the specific benefits and challenges in delivering a new service within community organisations is necessary to improve the translation and sustaining of memory-group programs.
\end{abstract}

Correspondence: Glynda Kinsella; g.kinsella@latrobe.edu.au

\section{A Comparison of Compensatory and Restorative Approaches to Memory Rehabilitation Post-Stroke: A Phase II Randomised Controlled Trial}

\author{
Toni D. Withiel, ${ }^{1}$ Dana Wong, ${ }^{1,2,3}$ Jennie L. Ponsford, ${ }^{1,3}$ Dominique A. Cadilhac, ${ }^{4,5}$ \\ Peter New, ${ }^{6}$ Tijana Mihaljcic ${ }^{1}$ and Renerus J. Stolwyk ${ }^{1,3}$ \\ ${ }^{1}$ Monash Institute of Cognitive and Clinical Neurosciences, School of Psychological Sciences, \\ Monash University, Melbourne, Australia \\ ${ }^{2}$ Monash Psychology Centre, Monash University, Melbourne, Australia \\ ${ }^{3}$ Monash-Epworth Rehabilitation Research Centre, Melbourne, Australia \\ 4 Translational Public Health Research, Stroke and Ageing Research Group, Monash University, \\ Melbourne, Australia \\ ${ }^{5}$ Stroke Division, The Florey Institute of Neuroscience and Mental Health, Heidelberg, Australia \\ ${ }^{6}$ Rehabilitation and Aged Care, Monash Health, Melbourne, Australia
}

$R$ ackground and aims: Memory deficits are common following stroke and have been independently linked to poorer rehabilitation outcomes. Two competing approaches have historically been used: computer-based restorative training and compensation strategies. The aim of the project was to compare 
the efficacy of computer restoration training and compensatory memory skills training following stroke.

Method: Randomised controlled design with three groups: computer restoration training, group-based compensatory memory skills training and waitlist control. Each intervention lasted 6 weeks. Blinded assessments occurred at baseline, post-intervention, and 6-week follow-up. The primary outcome measure was attainment on personalised memory goals using Goal Attainment Scaling. A range of secondary outcome measures were explored, including neuropsychological assessment of memory, subjective ratings of everyday memory failures and self-reported strategy use.

Results: Fifty-seven survivors of stroke with subjective memory complaints (median age 63 years, 56\% male, median time since stroke 27 months) were randomised. In the pilot data analysis $(n=40)$, participants allocated to the compensation memory intervention $(n=21)$ had significant improvement in individualised, memory specific goal attainment at post and follow up assessment relative to computer restorative and wait control participants $(F(1,40)=$ $8.28, p=<.01$, partial eta squared $=0.29)$. On secondary outcome measures, all groups self-reported reductions in everyday memory failures $(F(2,35)=$ $4.57, p=\leq .01$, partial eta squared $=0.21)$ and improved strategy use $(F(2,38)$ $=5.57, p=<.01$, partial eta squared $=0.23$ ) across time.

Conclusion: These preliminary pilot data indicate that compensatory memory rehabilitation improved functional memory on individualised goals following stroke, while restorative computer-based rehabilitation showed no significant effects compared with waitlist control.

Correspondence: Toni Withiel; toni.withiel@monash.edu

\section{Do People with Severe Traumatic Brain Injury Benefit from Making Errors? A Randomised Controlled Trial of Error-Based Learning and Errorless Learning}

Tamara Ownsworth, ${ }^{1}$ Jennifer Fleming, ${ }^{2}$ Robyn Tate, ${ }^{3}$ Elizabeth Beadle, ${ }^{1}$ Janelle Griffin, ${ }^{4}$ Melissa Kendall, ${ }^{5}$ Julia Schmidt, ${ }^{6}$ Amanda Lane-Brown, ${ }^{7}$ Mathilde Chevignard $^{8}$ and David. H. K. Shum ${ }^{1}$

${ }^{1}$ School of Applied Psychology and Menzies Health Institute Queensland, Griffith University, Mt Gravatt 4122, Australia

${ }^{2}$ School of Health and Rehabilitation Sciences, University of Queensland, St Lucia, Australia

${ }^{3}$ John Walsh Centre for Rehabilitation Research, Kolling Institute of Medical Research, The University of Sydney, Australia

${ }^{4}$ Princess Alexandra Hospital, Wooloongabba, Australia

${ }^{5}$ Acquired Brain Injury Outreach Service, Brisbane, Australia

${ }^{6}$ Faculty of Medicine, University of British Columbia, Vancouver, Canada

${ }^{7}$ Brain Injury Rehabilitation Unit, Liverpool Hospital, Sydney, Australia

${ }^{8}$ Rehabilitation Department for Children with Acquired Neurological Injury (INR-A), Hôpitaux de Saint Maurice, Saint Maurice, France

$\boldsymbol{R}$ ackground and aims: Errorless learning (ELL) and error-based learning (EBL) are commonly used in rehabilitation for people with traumatic brain injury (TBI). However, it is unknown whether making errors is beneficial in the learning process. This study aimed to compare the efficacy of ELL and EBL for improving self-regulation and self-awareness after severe TBI.

Method: A total of 54 adults $(79 \%$ male; $M$ age $=38.0$ years, $S D=13.4)$ with severe TBI were randomly allocated to ELL or EBL and received eight training sessions with an occupational therapist that involved meal preparation and other goal-directed activities. The primary outcome was total errors on the Cooking Task (near transfer) and secondary outcomes included the Zoo 
Map Test (far transfer), Patient Competency Rating Scale and measures of psychosocial functioning (e.g., mood, independence, work).

Results: Controlling for baseline performance, EBL participants made significantly fewer errors on the Cooking Task at post-intervention than ELL participants $\left(F=5.47, p=.023, \eta \rho^{2}=.10\right)$. EBL participants also demonstrated greater self-awareness than ELL participants $\left(p=.028, \eta \rho^{2}=.10\right)$. There were no significant differences on the Zoo Map Test or psychosocial outcomes $(p>.05)$; however, anxiety levels were lower at post-intervention relative to baseline for ELL participants only.

Conclusions: EBL is more effective than ELL for enhancing skills generalisation on tasks related to training and improving self-awareness. However, EBL has no significant advantage over ELL for facilitating skills generalisation on tasks unrelated to training or improving broader psychosocial outcomes. The longer-term effects of each intervention will be examined at 6-month follow-up.

Correspondence: Tamara Ownsworth; t.ownsworth@griffith.edu.au

\title{
Type of Cerebrovascular Accident and Influence on Rehabilitation Planning and Outcomes
}

\author{
Elly Williams, ${ }^{1}$ Janet Wagland ${ }^{1}$ and Angelita Martini $^{2}$ \\ ${ }^{1}$ Brightwater Care Group, Perth, Australia \\ ${ }^{2}$ University of Western Australia
}

$\boldsymbol{R}$ ackground and aims: People with an acquired brain injury (ABI) present to post-acute rehabilitation with complex disability. Therefore, it is important to accurately identify potential for rehabilitation on initial assessment to optimise client outcomes and use of health resources. Type of ABI can have a large influence on severity of injury and the areas in which rehabilitation gains can be made. This study assessed and compares the functional and cognitive gains made by clients with left/right, haemorrhagic/ischaemic CVA diagnosis 6 months after entering a post-acute rehabilitation service.

Method: Mayo-Portland Adaptability Inventory-4 (MPAI-4) client assessments at admission, 3 monthly review and discharge were analysed using STATA statistical software. The retrospective de-identified data from 60 clients was stratified into primary diagnosis groups, being: CVA Haemorrhagic right brain $(n=8)$, haemorrhagic left brain $(n=12)$, ischaemic right brain $(n=$ $16)$ and ischaemic left brain $(n=24)$. Non-parametric statistical analysis was used to compare MPAI-4 scores from admission to 6 months post admission. Results: Clients with an ischaemic right brain CVA presented with the highest impairment level and made no significant gains. Clients with a haemorrhagic right brain CVA had the most improvement over the 6 month period. Those with ischaemic left brain CVA presented with the lowest impairment levels and made significant gains $(p=.0097)$. Clients with haemorrhagic left brain CVA made significant gains in participation $(p=.0042)$.

Conclusions: The MPAI- 4 confirms type of CVA can inform rehabilitation planning for clients entering post-acute rehabilitation and enables insight into what areas of rehabilitation should be targeted.

Correspondence: Elly Williams; elly.williams@ brightwatergroup.com 


\title{
Functional Outcomes from a Specialised Acquired Brain Injury Community Rehabilitation Service - Evaluating a New Model of Care
}

\author{
Michelle Farquhar, ${ }^{1}$ Natasha A. Lannin ${ }^{1,2}$ and Jacqui Morarty ${ }^{1}$ \\ ${ }^{1}$ Acquired Brain Injury Unit, Caulfield Hospital, Melbourne, Australia \\ ${ }^{2}$ Department of Occupational Therapy, LaTrobe University, Melbourne, Australia
}

$\boldsymbol{R}$ ackground and objectives: Approximately one third of adults with acB quired brain injury (ABI) experience significant difficulties with everyday living after discharge. Access to community-based rehabilitation is essential, to maximise independent functioning in the context of peoples' health and daily lives. Historically, community rehabilitation has been limited by admission timeframes, client accommodation and catchment areas. Our specialist $\mathrm{ABI}$ community service is without admission timeframes, serves a state-wide catchment and can access young people residing in residential care. This model enables therapy goals to be addressed across the lifespan.

The effectiveness of a new specialised state-wide, life-long community rehabilitation service will be reviewed, focussing on patterns of independence within occupational performance and community integration, as well as rehabilitation length of stay and community cost.

Method: A number of outcome measures were implemented in early 2016 on admission and discharge: Community Integration Questionnaire, Service Outcome Scale, Mayo Portland Adaptability Inventory, Functional Autonomy Measurement System and the Care And Needs Scale.

Results: Admission, monitoring and discharge results were compared. At time of writing, $n=19$ data sets showed that all measures reflecting community integration and occupational performance improved. Access to service and support knowledge improved. Cost-related data indicated a clear impact, while 'discharge to monitoring' enabled goals to be reviewed and re-set as appropriate.

Conclusions: The implementation of key outcome measures in a specialised community rehabilitation service enabled intervention, as well as the client's experience, to be measured, providing valuable information for the future of this model of care.

Correspondence: Michelle Farquhar; m.farquhar@alfred.org.au

\section{An Inpatient Breakfast Program Increases Participation in Brain Injury Rehabilitation: A Pre and Post Study}

\author{
Michelle Quick, ${ }^{1}$ Laura Jolliffe ${ }^{2}$ and Natasha Lannin ${ }^{1,2}$ \\ ${ }^{1}$ Occupational Therapy Department, Alfred Health, Caulfield, Australia \\ ${ }^{2}$ Discipline of Occupational Therapy, School of Allied Health, College of Science, Health and Engi- \\ neering, La Trobe University, Bundoora, Australia
}

$\boldsymbol{R}$ ackground and aims: Meal preparation is an important daily routine

3 however in the inpatient hospital setting it can be difficult to provide an environment where patients can initiate and participate making breakfast unsupervised. To increase independence in morning routines, an ABI Rehabilitation Unit in a Melbourne Metropolitan hospital initiated a "do it yourself" (DIY) breakfast program.

Method: A literature review was first completed to develop the content and policies for an unsupervised, hospital-based breakfast program. Data were collected on number of inpatients initiating and making their own breakfast both pre and 6 months post commencing the program using observational audit methods. In addition to patient participation, we also recorded Functional 
Independence Measure (FIM) and Functional Autonomy Measurement System (SMAF) scores to describe the functional abilities of all inpatients during the study period.

Results: Prior to implementation of the DIY breakfast program there were limited opportunities to independently prepare your own breakfast and engage in a morning routine that was contextually meaningful for patients. Six months following implementation of the breakfast program, there was a significant increase in participation of self-directed breakfast from $0 \%$ of eligible patients (baseline) to 64\% (6-months post-implementation) with concurrent increase in engagement in occupation during hospitalisation after ABI (Z-score -3.722 , $p$-value .0002).

Conclusions: An unsupervised breakfast program increased self-directed participation breakfast preparation during hospitalisation in adults with severe brain injury. Providing meal preparation opportunities within a self-directed program may increase independence and could be considered for implementation by other services

Correspondence: Michelle Quick; m.quick@alfred.org.au

\title{
Music Interventions for Acquired Brain Injury: Findings from an Updated Cochrane Review
}

\author{
Jeanette Tamplin, ${ }^{1,2}$ Wendy Magee, ${ }^{3}$ Imogen Clark ${ }^{1,2}$ and Joke Bradt ${ }^{4}$ \\ ${ }^{7}$ National Music Therapy Research Unit, University of Melbourne, Melbourne, Australia \\ ${ }^{2}$ Music Therapy Department, Royal Talbot Rehabilitation Centre - Austin Health, Melbourne, Australia \\ ${ }^{3}$ Boyer College of Music and Dance, Temple University, Philadelphia, USA \\ ${ }^{4}$ Department of Creative Arts Therapies, Drexel University, Philadelphia, USA
}

$\boldsymbol{R}$ ackground and aims: This Cochrane review update examined the effects of music interventions and standard care versus standard care alone or standard care combined with other therapies for adults with non-degenerative acquired brain injury (ABI). Primary outcomes were gait and upper extremity function. Secondary outcomes were communication, cognition, mood and emotions, social skills, pain, behavioural outcomes, activities of daily living, and adverse events.

Method: We searched electronic databases, dissertation and specialist music databases, and hand-searched grey literature. All randomised controlled and controlled clinical trials with quasi-randomised or systematic methods of treatment allocation were included, in any language, published and unpublished.

Results: We included 29 trials with 775 participants. Meta-analyses indicated that music interventions may improve gait velocity, stride length (affected side), gait cadence, stride symmetry, general gait, and timing of upper extremity function. Using a rhythmic auditory stimulus embedded in music may lead to greater improvements in gait velocity than using rhythmic auditory stimulus without music e.g., metronome. Interventions delivered by a credentialed music therapist generated greater improvements than interventions delivered by other professionals. Music interventions may also improve quality of life and overall communication with beneficial effects in naming and repetition.

Conclusions: Rhythm-based interventions remain important for movement rehabilitation in ABI. Future research should examine music intervention effects on cognition. Measures used for mood outcomes require greater uniformity and improved reporting. Across all domains, improved agreement on the core outcomes examined will enable more comprehensive meta-analyses and more accurate evaluation of the effectiveness of music interventions.

Correspondence: Jeanette Tamplin; jeanette.tamplin@unimelb.edu.au 


\section{Brain Stimulation, Aging and Cognition}

Melanie R. L. Emonson, ${ }^{1}$ Paul B. Fitzgerald, ${ }^{1}$ Nigel C. Rogasch ${ }^{2}$ and Kate E. Hoy ${ }^{1}$

${ }^{1}$ Monash Alfred Psychiatry Research Centre, The Alfred and Monash University

${ }^{2}$ Brain and Mental Health Laboratory, School of Psychological Sciences and Monash Biomedical Imaging, Monash University, Australia

$\boldsymbol{B}$ ackground and aims: Mild Cognitive Impairment (MCI) may repreB sent a prodromal stage of neurodegeneration. As such it is important to characterise the neurobiological changes in order to develop efficacious interventions. Combining transcranial magnetic stimulation with electroencephalography (TMS-EEG) can provide information on the neural plasticity changes present. The current study used TMS-EEG to characterise the differences in neural plasticity responses, and its relation to cognitive performance in younger, older and MCI participants.

Method: 20 healthy younger, 20 healthy older and 10 adults with MCI completed a neuropsychological battery, computerised cognitive tasks and TMSEEG (50 single pulses), prior to delivery of transcranial direct current stimulation (tDCS). 20 minutes $1 \mathrm{~mA}$ tDCS was administered to the left dorsolateral prefrontal cortex (DLPFC), with TMS-EEG and cognitive tasks repeated across multiple time-points post-tDCS.

Results: Preliminary analyses show significantly reduced amplitude of early peak TMS responses in MCI $(p<.05)$. In older adults early responses correlated with verbal learning and memory and attention tasks $(p<.05)$. There was a significant effect of time for the N120 amplitude, with younger and older adults showing a reduction post-tDCS $(p$ 's $<.05)$. In older adults, the N120 amplitude at T20 positively correlated with reaction time on the n-back task post-tDCS $(p<.05)$.

Conclusions: Reduced early responses in MCI suggests poor synchrony of neural activity or disrupted functional connectivity, which may contribute to reduced memory functioning. Reduction in N120, a marker of inhibition, in younger and older, but not MCI may indicate poor ability to modulate inhibition following tDCS.

Correspondence: Melanie Emonson; melanie.emonson@monash.edu

\section{Concurrent Session 2}

\section{Does Age Affect Ratings of Post-Concussion Symptoms in a Paediatric Population?}

Gabriella Flaks, ${ }^{1,2}$ Michael Takagi, ${ }^{1,2}$ Stephen Hearps, ${ }^{1}$ Franz Babl, ${ }^{1,3,4}$ Silvia Bressan, ${ }^{1,5}$ Katherine Truss, ${ }^{1,2}$ Gavin Davis, ${ }^{1}$ Alison Crichton, ${ }^{1,2,8}$ Celia Godfrey, ${ }^{1}$ Cathriona Clarke, ${ }^{1}$ Melissa Doyle, ${ }^{1,2}$ Vanessa Rausa, ${ }^{1,2}$ Kevin Dunne ${ }^{1,4,6}$ and Vicki Anderson ${ }^{1,2,4,7}$

${ }^{7}$ Murdoch Childrens Research Institute, Melbourne, Victoria, Australia

${ }^{2}$ Melbourne School of Psychological Sciences, University of Melbourne, Victoria, Australia

${ }^{3}$ Emergency Department, Royal Children's Hospital, Melbourne, Victoria, Australia

${ }^{4}$ Department of Paediatrics, University of Melbourne, Victoria, Australia

${ }^{5}$ Department of Women's and Children's Health, University of Padova, Italy

${ }^{6}$ Department of Rehabilitation Medicine, Royal Children's Hospital, Melbourne, Victoria, Australia

${ }^{7}$ Psychology Service, The Royal Children's Hospital, Melbourne, Australia

${ }^{8}$ Victorian Pediatric Rehabilitation Service, Monash Children's, Melbourne, Australia ples are treated as a unified group, irrespective of age. Cut-off scores have 
not yet been researched across age group, and at present, the same benchmark clinical risk score, is applied to ages 5-18. We investigated whether postconcussion symptom (PCS) recovery differs by age group; whether the proportion of children with delayed recovery differs across age groups at 2-weeks post-concussion; and to explore factors that predict recovery. Our research affords the opportunity to determine whether age is a risk factor for greater PCS following concussion.

Method: This study was a prospective, longitudinal observational study conducted between July 2013 and November 2015 at a state-wide tertiary paediatric hospital. The sample consisted of 133 children aged 5-18 years who had sustained a concussion within 48 hours of presentation to the Emergency Department. PCS were assessed using the Post-Concussion Symptom Inventory, acutely, 1-4 days post-injury and 2 weeks post-injury. Concussion recovery 2 -weeks post-injury was defined by a validated cut-off score.

Results: Forty per cent of children were symptomatic at 2-weeks post-injury, with $36 \%$ of children aged 5-7, 43\% aged 8-12 and 38\% aged 13-18, symptomatic at 2 weeks. A chi-square analysis revealed no significant relationship between PCS recovery and age group. A logistic regression showed that predictors of recovery (age group, parental psychological distress, child PTSD symptoms and child behaviour) did not distinguish between children who recovered and those who remained symptomatic 2 -weeks post-concussion.

Conclusions: These results have implications for children and adolescents post-concussion, directly impacting concussion diagnosis, treatment, and assessment.

Correspondence: Michael Takagi; michael.takagi@mcri.edu.au

\title{
Do Baseline Factors Tell Us which Children are Likely to Experience Worse Post-Concussion Fatigue?
}

Alison Crichton, ${ }^{1,2}$ Michael Takagi, ${ }^{1,2}$ Stephen Hearps, ${ }^{1}$ Franz Babl, ${ }^{1,3,4}$ Ed Oakley, ${ }^{1,3}$ Silvia Bressan, ${ }^{1,5}$ Katherine Truss, ${ }^{1,2}$ Gavin Davis, ${ }^{1}$ Celia Godfrey, ${ }^{1}$ Cathriona Clarke, ${ }^{1}$ Melissa Doyle, ${ }^{1,2}$ Gabriella Flaks, ${ }^{1,2}$ Vanessa Rausa, ${ }^{1,2}$ Kevin Dunne ${ }^{1,4,6}$ and Vicki Anderson $1,2,4,7$

\author{
${ }^{7}$ Murdoch Childrens Research Institute, Melbourne, Victoria, Australia \\ ${ }^{2}$ Melbourne School of Psychological Sciences, University of Melbourne, Victoria, Australia \\ ${ }^{3}$ Emergency Department, Royal Children's Hospital, Melbourne, Victoria, Australia \\ ${ }^{4}$ Department of Paediatrics, University of Melbourne, Victoria, Australia \\ ${ }^{5}$ Department of Women's and Children's Health, University of Padova, Italy \\ ${ }^{6}$ Department of Rehabilitation Medicine, Royal Children's Hospital, Melbourne, Victoria, Australia \\ ${ }^{7}$ Psychology Service, The Royal Children's Hospital, Melbourne, Australia \\ ${ }^{8}$ Victorian Pediatric Rehabilitation Service, Monash Children's, Melbourne, Australia
}

$\boldsymbol{R}$ ackground and aims: Fatigue is one of the most common complaints after child concussion, with up to $47 \%$ of symptomatic children reporting significant fatigue (Barlow, 2016). However, others, (Iverson et al., 2015) reported fatigue in healthy adolescents (particularly females), suggesting concussion symptoms may relate to pre-existing conditions. Therefore, key risk factors might be able to be identified that place children at risk of experiencing post-concussion fatigue. We explore pre-injury child risk factors for worse fatigue after concussion.

Method: A substudy of TakeCARe, this prospective longitudinal observational study of children presenting to a tertiary paediatric hospital within 48 hours of concussion, provides follow up at 3 months post-injury. The Pediatric Quality of Life Inventory Multidimensional Fatigue Scale was the primary outcome (parent ratings, completed by 111 parents). The PedsQL MFS measures 
fatigue across three dimensions including lack of physical energy (general fatigue), sleep/wake cycle disruption (sleep/rest fatigue), and depleted mental endurance/attention (cognitive fatigue). Clinical fatigue was defined as fatigue two standard deviations above healthy control data. Baseline data was collected as per protocol.

Results: At 3 month follow-up 17\% of children reported significant fatigue. Regression analysis demonstrated baseline characteristics poorly predicted post-concussion fatigue. However, a significant prediction for sleep/rest fatigue symptoms from age $(\mathrm{p}<.05)$. Other demographic factors were non-significant. We explored other pre-injury risk factors for poor fatigue prognosis.

Conclusions: It is difficult to identify children at risk of post-concussion fatigue. Injury, and post-injury adjustment and symptoms are areas for future research to identify those children in need of clinical follow up.

Correspondence: Alison Crichton; ali.crichton@mcri.edu.au

\section{The Structure and Protocol of the Take CARe Study}

Michael Takagi, ${ }^{1,2}$ Alison Crichton, ${ }^{1,2}$ Stephen Hearps, ${ }^{1}$ Franz Babl, ${ }^{1,3,4}$ Ed Oakley, ${ }^{1,3}$ Silvia Bressan, ${ }^{1,5}$ Katherine Truss, ${ }^{1,2}$ Gavin Davis, ${ }^{1}$ Celia Godfrey, ${ }^{1}$ Cathriona Clarke, ${ }^{1}$ Melissa Doyle, ${ }^{1,2}$ Gabriella Flaks, ${ }^{1,2}$ Vanessa Rausa, ${ }^{1,2}$ Kevin Dunne ${ }^{1,4,6}$ and Vicki Anderson 1,2,4,7

${ }^{1}$ Murdoch Childrens Research Institute, Melbourne, Victoria, Australia

${ }^{2}$ Melbourne School of Psychological Sciences, University of Melbourne, Victoria, Australia

${ }^{3}$ Emergency Department, Royal Children's Hospital, Melbourne, Victoria, Australia

${ }^{4}$ Department of Paediatrics, University of Melbourne, Victoria, Australia

${ }^{5}$ Department of Women's and Children's Health, University of Padova, Italy

${ }^{6}$ Department of Rehabilitation Medicine, Royal Children's Hospital, Melbourne, Victoria, Australia

${ }^{7}$ Psychology Service, The Royal Children's Hospital, Melbourne, Australia

${ }^{8}$ Victorian Pediatric Rehabilitation Service, Monash Children's, Melbourne, Australia

ntroduction and aims: The majority of children who sustain a concussion recover spontaneously within 1-2 weeks. However, a substantial minority of children suffer from a constellation of post-concussive symptoms (PCS) that can persist for more than 1-month post-injury and include physical, cognitive, behavioural, and emotional changes. Those affected can develop significant disability, which may diminish their quality of life. Heterogeneous and at times conflicting results have been published on the factors that predict which children are at a high risk for developing long-lasting PCS. The aim of the Take CARe (Concussion Assessment and Recovery Research) study is to examine which presenting clinical features predict rapid recovery, and which predict prolonged recovery.

Procedure: The Take CARe project is a prospective, longitudinal study based at the Royal Children's Hospital and the Murdoch Childrens Research Institute in Melbourne, Australia. We recruit children aged 5-18 who present to the ED with a concussion and follow them up at 1-4 days, 2-weeks, 1-month, and 3-months post-injury. Data collected includes: clinical, demographic, injuryrelated, mental health, psychological, blood biomarkers, MRI, cognitive, and economic.

Results: Since July of 2013, 378 participants have been recruited, data collection will continue until mid-2017.

Discussion: We will discuss the procedures and structure for Take CARe, as well as common clinical issues. This includes: definitional issues (e.g., consistent definition of PCS); identifying the best instruments to assess symptoms; and deriving clinically meaningful information.

Correspondence: Michael Takagi; michael.takagi@mcri.edu.au 


\title{
Family Outcomes and TBI: A Pilot Investigation Using the Family Outcome Measure
}

\author{
Christine Migliorini, Libby Callaway and Sophie Moore \\ Department of Occupational Therapy, Monash University, Melbourne, Australia
}

\begin{abstract}
$B$ ackground and aims: Research focused on family members (FMs) demonstrates that their mental health directly affects, and is affected by, the outcomes of their relative with TBI. However, few researchers have explored ongoing resilience and sustainability of families especially several years post-injury. This study aimed to examine family outcomes and factors that may impact on those outcomes, in the years following the occurrence of the TBI.

Methods: Pilot cross-sectional design with adult FMs $(n=38)$ nominated by relatives with moderate to severe TBI $(n=38)$ who were living in the community. Measures included Family Outcome Measure and Health of the Nations Outcome Scale - ABI completed by FM; and Roles Checklist completed by the relative with TBI.

Results: Sustainability of family support was positively associated with increased family closeness and increased FM resilience, plus lower levels of challenging behaviour and older age of the relative with TBI. FM resilience was positively associated with better FM coping, lower FM feelings of burden and improved service support. Resilience was also associated with less mental health symptoms but higher physical comorbidity of the relative with TBI. Feelings of burden were lower in FMs when the relative resided in SSA; however, place of residence was not associated with FM coping, FM resilience or family closeness.

Conclusions: This study begins to address the knowledge gap regarding personal, environmental and injury factors that may influence family outcomes. It also provides insights into the adjustment of family members following TBI.

Correspondence: Christine Migliorini; christine.migliorini@monash.edu
\end{abstract}

\section{Increasing Family Engagement in Brain Injury Rehabilitation: What are the Active Ingredients for Success?}

\author{
Alinka Fisher, ${ }^{1}$ Michelle Bellon, ${ }^{1}$ Sharon Lawn, ${ }^{2}$ Sheila Lennon ${ }^{3}$ and McKay \\ Sohlberg ${ }^{4}$ \\ ${ }^{1}$ Department of Disability and Community Inclusion, Flinders University, Adelaide, Australia \\ ${ }^{2}$ Department of Psychiatry, Flinders University, Adelaide, Australia \\ ${ }^{3}$ Discipline of Physiotherapy, Flinders University, Adelaide, Australia \\ ${ }^{4}$ Communication Disorders \& Sciences, University of Oregon, United States of America
}

\begin{abstract}
$\boldsymbol{B}$ ackground and aims: The current focus on increasing the involvement $\mathcal{B}$ of family members in the rehabilitation process is driven by economic and therapeutic forces. Within the current financial climate, where resources are limited, there is the need to develop and evaluate supports that can be implemented by natural everyday support people. Family members are ideally positioned for this role as they are emotionally invested in the individual's outcomes, and report a desire for further practical hands-on collaboration. Furthermore, family involvement has been linked to improved rehabilitation outcomes for individuals with ABI. The question is then, how do we facilitate the increased competence of family caregivers as facilitators in the rehabilitation process?
\end{abstract}

Method: The literature was reviewed to identify the active ingredients behind family engagement in brain injury rehabilitation, including effective 
professional-family relationships, and important factors in the delivery of education underpinned by evidence-based practice.

Results: This research introduces the Family-directed Approach to Brain injury management (FAB) model. The FAB model, which is based on principles of hope, family expertise, education/ skill building, and family-directed intervention, provides a theoretical framework for supporting family caregivers as facilitators of change. Recommendations are presented to guide professionals in this process, encouraging them to reflect on the importance of their therapeutic relationships and their capacity to positively impact rehabilitation outcomes beyond the corporate aspects of health care.

Conclusion: With the current shift towards greater family collaboration, the FAB model defines potential active ingredients and provides a theoretical framework to guide treatment implementation.

Correspondence: Alinka Fisher; Alinka.Fisher@flinders.edu.au

\title{
"Now I can pat myself on the back, we stopped that behaviour from going south?": A Pilot Study Of The Family-Directed Behaviour Support (FDBS) Program for Individuals with Brain Injury.
}

\author{
Alinka Fisher, ${ }^{1}$ Michelle Bellon, ${ }^{1}$ Sharon Lawn ${ }^{2}$ and Sheila Lennon ${ }^{3}$ \\ ${ }^{1}$ Department of Disability and Community Inclusion, Flinders University, Adelaide, Australia \\ ${ }^{2}$ Department of Psychiatry, Flinders University, Adelaide, Australia \\ ${ }^{3}$ Discipline of Physiotherapy, Flinders University, Adelaide, Australia
}

$\boldsymbol{R}$ ackground and aims: A Family-Directed Behaviour Support (FDBS) program was developed to increase the competency of family caregivers in supporting behaviour change in relatives with ABI, addressing unmet information and practical support needs and reducing families' dependency on specialised services, which are currently limited. The FDBS program is based on a positive behaviour support framework and consists of a 4-week education phase, followed by six individualised sessions. The aim of this study was to examine the acceptability of the program and gather data to refine its development.

Method: Two family caregivers of adults with ABI completed the 10-week program. They were responsible for collecting and analysing observation data, implementing and monitoring strategies. Multiple assessments were conducted pre and post intervention, and at 3-months follow-up using the Overt Behaviour Scale, Caregiver Appraisal Scale, a behaviour tally and purpose-developed confidence questionnaire. Semi-structured interviews and questionnaires were also carried out following both the education phase and individualised sessions to gather feedback regarding participants' experience of the program.

Results: Participants reported a reduction in frequency and intensity of BOC, high satisfaction regarding participation, and increased confidence in identifying strategies and responding to behaviours of concern. No significant change was reported in levels of caregiver burden. The program was further refined according to participant feedback and facilitator reflections.

Conclusion: These findings suggest the FDBS program may be an acceptable approach to increase competence of family caregivers in supporting behaviour change in relatives with $\mathrm{ABI}$, providing recommendations for a larger scale feasibility study.

Correspondence: Alinka Fisher; Alinka.Fisher@flinders.edu.au 


\title{
Taking Up the Challenge: Improving the Management of Challenging Behaviours in Patients with a Traumatic Brain Injury in the Acute Setting
}

Felicity Jenkinson

Department of Allied Health/Neurosurgery, Southern Adelaide Local Health Network, Adelaide, Australia

\begin{abstract}
$\boldsymbol{R}$ ackground: Review of the management of traumatic brain injury (TBI) patients at Flinders Medical Centre (FMC) Neurosurgery identified areas for improvement in regards to managing challenging behaviours. Many of these patients exhibited behaviours triggering "Code Blacks" and the subsequent administering of pharmacological and / or physical restraint.

Methods: An audit of 38 case notes of patients admitted to FMC with a severe TBI was completed to investigate amount of code blacks, medications administered, restraints used, documentation of behaviour and demographics. Best practice was identified using a literature search and expert opinion, and a protocol was developed which outlined the non-pharmacological and pharmacological management of challenging behaviours for TBI patients in the acute phase. A repeat audit was completed to review outcomes.

Results: Results demonstrated between August 2012 and December 2014, $18.4 \%$ of admissions required "Code Blacks" (7/38). Of these patients, $84 \%$ required some form of restraint $(50 \%$ chemical, $34 \%$ physical and $29 \%$ physical and chemical). Variation in medications was evident and descriptions of behaviour were unmeasurable and non-descript when behaviour became challenging enough to require restraint. Since trialling the protocol, "Code Blacks" reduced by $4 \%$ and multiple 'Code Blacks' by $38 \%$. The total number of restraints (chemical and physical) reduced by $51 \%$.

Conclusion: Implementation of the TBI- challenging behaviours in the acute phase protocol has reduced the requirement for restraints and variation of medications provided to TBI patients in the acute setting at Flinders Medical Centre.
\end{abstract}

Correspondence: Felicity Jenkinson; felicity.jenkinson@sa.gov.au

\section{Concurrent Session 3 - How to Session}

\section{Developing Apps for Mental Health}

David Bakker

Monash University, Melbourne, Australia

C ynopsis of session: Smartphones are ubiquitous, interactive, flexible tools that are being used for an increasing number of health purposes. This session will offer an insight into how smartphone apps can be developed by researchers and clinicians. Particular attention will be paid to addressing the unique needs that people with ABI or mental health issues have. Every step from initial design to software development to dispersion and refinement will be covered. Additionally, attendees will gain insight into how to best use apps in their practice. 


\title{
Road to Recovery - Mental Health Assessment and Treatment for People with Brain Impairment in the Criminal Justice System
}

\author{
Dani Ashley, Erik Meurs and Ming-Yun Hsieh \\ Mobile Forensic Mental Health Service, Forensicare
}

Cynopsis of session: People with brain impairment (whether due to neu$\mathcal{N}$ rological, psychiatric or medical conditions) are over-represented in the criminal justice system and are often vulnerable during their time in custody. They often also have significant mental health assessment and treatment needs that can be difficult to meet in a custodial environment. This session will present a multi-disciplinary assessment and therapeutic approach being utilised in working with prisoners with comorbid mental health difficulty and brain impairment. We will provide a broad overview of criminal justice system and the potential legal and sentencing implications for people with brain impairment. We will describe how the principles of neurorehabilitation and the mental health recovery model have helped shape our work with individuals with comorbid brain impairment and mental health difficulty. This session will be of interest to clinicians working with clients who are currently (or at risk of being) involved in the criminal justice system. De-identified case studies will be incorporated throughout the session to illustrate how we strive to address high risk and complex mental health presentations in custodial environments and how to work towards a better outcome upon clients' release back to the community.

\section{Concurrent Session 4}

\section{"You do not live in a nursing home; you exist": Experiences of Young People with Acquired Brain Injury (ABI) Entering, Living in and Leaving Residential Aged Care in Australia}

\author{
Lucy Knox, ${ }^{1,2}$ Sandra Barry ${ }^{1,2}$ and Jacinta Douglas ${ }^{1,2}$ \\ ${ }^{1}$ Living with Disability Research Centre, La Trobe University \\ ${ }^{2}$ Summer Foundation, Victoria
}

$B$ ackground and aims: Previous research has highlighted that living in residential aged care (RAC) is associated with a range of negative implications for adults with ABI. This study sought to develop an understanding of the experience of entering and living in RAC from the perspective of young people who are living or have lived in RAC and their family members.

Method: Data was drawn from written and verbal submissions made to the Senate Inquiry into the adequacy of existing residential care arrangements available for young people with severe physical, mental or intellectual disabilities in Australia. Sixty-four submissions from young people with ABI and their parents, siblings, and spouses were analysed using thematic analysis. Results: Themes centred around the distinct processes of entering, residing in, and living again upon exiting RAC. Multiple pathways into RAC were described. However, for both adults with ABI and family members, entry to RAC reflected a choice constrained by information, funding and alternatives. Life in RAC was characterised across a range of dimensions (care relationships, risk tolerance, feeling of belonging, sense of hope) which shaped the person's sense of self. Participants who had exited RAC described being able to live 
again as they rediscovered valued roles and re-established a positive sense of self.

Conclusions: These findings augment existing literature by providing insight into the complex interaction that exists between the experience of life in RAC, family relationships, and self-conceptualisation after ABI. Findings highlight the role clinicians can play in assisting individuals and families in this process.

Correspondence: Lucy Knox; L.Knox@ latrobe.edu.au

\title{
Technology, Housing and Outcomes: A Scoping Review
}

\author{
Natasha Layton,, ${ }^{1,2}$ Jacinta Douglas ${ }^{1,2}$ and Di Winkler ${ }^{1,2}$ \\ ${ }^{1}$ Summer Foundation, Blackburn, Australia \\ ${ }^{2}$ Living with Disability Research Centre, School of Allied Health, La Trobe University, Bundoora, \\ Australia
}

\begin{abstract}
$\boldsymbol{R}$ ackground and aims: If individuals living with physical and cognitive impairment are able to control their environments, the experience of disability diminishes. Technology integrated into buildings ('integrated technology') is one aspect of housing design with the potential to achieve this. The rapidly developing technology market and changing disability policy context indicate a need to review technologies for people living in specialist disability accommodation (SDA). The aim of this study was to investigate the use of technology within housing and the measurement of its outcomes.

Method: A scoping review was conducted, targeting smart homes and communication technology evaluations in the ABI, general disability, ageing, engineering and housing literature.

Results: Nine reviews, representing over 450 studies, reported varied results for a range of primary (person-focussed) and secondary (system level) outcome domains. Moderate evidence exists for focal technology products and discrete populations, with weaker but promising evidence for many aspects of technology-within-housing. A small number of studies without significant results, and some studies with a focus on user experience, suggest a range of user preferences and ethical considerations which impact on effectiveness. Methodologically, significant issues exist with technology definitions, scope, currency and with outcome measurement.

Conclusion: There is significant potential for delivery of a range of outcomes with appropriately targeted integrated technologies. Frameworks for ethical provision, within a 'technology continuum' are proposed. The scoping review informs the selection of outcome measures and identification of technology types in the design of future studies and housing builds.
\end{abstract}

Correspondence: Natasha Layton; natasha.layton@summerfoundation.org.au

\section{Transitional Living Services for Adults with an Acquired Brain Injury: A Scoping Review}

\author{
Anna Potter, ${ }^{1,2}$ Danielle Sansonetti, ${ }^{1,2}$ Kate D'Cruz ${ }^{3}$ and Natasha Lannin ${ }^{1,3}$ \\ ${ }^{1}$ Occupational Therapy Department, Alfred Health, Caulfield, Australia \\ ${ }^{2}$ Acquired Brain Injury Rehabilitation Centre, Alfred Health, Caulfield, Australia \\ ${ }^{3}$ Discipline of Occupational Therapy, School of Allied Health, College of Science, Health and Engi- \\ neering, La Trobe University, Bundoora, Australia
}

$\boldsymbol{R}$ ackground and objectives: Transitional living service (TLS) programs for adults with a brain injury are an important part of rehabilitation. However, considerable variability exists in the design and structure of these services across Australia, with limited research to guide the development of a 
program based on best evidence. A scoping literature review was completed to answer the question 'What is known about transitional living service programs for adults with an acquired brain injury?'

Method: A comprehensive and systematic approach to searching was undertaken and documented for replicability using the PRISMA checklist. Four electronic databases were systematically searched (CINAHL, MEDLINE, PsycINFO, and OTseeker), followed by a grey literature search (from 19962015). An inductive approach was used to thematically analyse data retrieved from the included studies.

Results: Thirteen articles were included in the final review. Themes that emerged from the literature will be presented, including: the types of residents using TLS programs, the subjective experience of residents and staff, intervention approaches, program staffing, and program outcomes.

Conclusions: The research reviewed supports the use of TLS programs to maximise functional independence and community integration of individuals with an acquired brain injury. Findings have been synthesised into clinical practice recommendations to support implementation of a new TLS program at Alfred Health, and include: use multiple outcome measures, implement collaborative goal setting, support generalisation of skills learnt in the TLS to the home environment, and eligibility criteria for accessing a TLS program to include individuals across all phases of recovery.

Correspondence: Danielle Sansonetti; D.Sansonetti@alfred.org.au

\section{Exploring the Evidence for Extended Rehabilitation for Adults with Very Severe ABI: Results from a Scoping Review}

Lucy Knox ${ }^{1,2}$ and Jacinta Douglas ${ }^{1,2}$

${ }^{1}$ Living with Disability Research Centre, La Trobe University

${ }^{2}$ Summer Foundation, Victoria

$\boldsymbol{R}$ ackground and aims: Research suggests that many adults with very severe acquired brain injuries (ABI) do not receive adequate rehabilitation. Poor access to rehabilitation not only constrains recovery but can also lead to inappropriate and lengthy nursing home admission. This review aimed to map the existing literature relating to rehabilitation programs for adults experiencing a prolonged recovery after very severe $\mathrm{ABI}$.

Method: A comprehensive literature search was undertaken, including systematic searching of databases (MEDLINE, CINAHL, PsycINFO, AMED, Web of Science), grey literature, and hand searching. To be eligible for inclusion, studies had to report on (a) prolonged or 'slow-stream' rehabilitation interventions for (b) adults with very severe $\mathrm{ABI}$ and complex support needs, and describe (c) the outcomes of the intervention.

Results: From an initial total of 17,829 citations, 19 records were retained for inclusion in this review. Data extraction focused on three categories relating to (i) participant characteristics, (ii) program information, and (iii) study design and findings. There was significant diversity across this literature, both in describing and defining the population and the nature of the interventions provided. However, findings suggested that extended rehabilitation programs assisted participants to live in more independent, home-like environments and contributed towards significant savings in their lifetime care costs.

Conclusions: The findings of this review suggest that specialised rehabilitation can maximise the independence and participation of adults with very severe ABI. Further collaboration and advocacy is required to ensure that adults 
with very severe injuries have access to appropriately timed and individuallytailored intervention programs.

Correspondence: Lucy Knox; L.Knox@latrobe.edu.au

\title{
Return to Driving after Traumatic Brain Injury: A Systematic Review of Rates and Timing and Influencing Factors
}

\author{
Janelle Griffin ${ }^{1}$ and Jennifer Fleming ${ }^{1,2}$ \\ ${ }^{1}$ Occupational Therapy Department, Princess Alexandra Hospital, Brisbane, Australia \\ ${ }^{2}$ The University of Queensland, School of Health and Rehabilitation Sciences, Brisbane, Australia
}

\begin{abstract}
$\boldsymbol{B}$ ackground and objectives: Many people want to return to driving following a traumatic brain injury yet the rate and timing for when this occurs varies. The aim of this systematic review was to determine the proportion of people that return to driving following traumatic brain injury and the timeframe that the return to driving occurs, and to identify the influencing factors.

Method: Eight databases were searched for quantitative studies reporting on return to driving for people with traumatic brain injury. The two authors independently reviewed studies against inclusion criteria. Key findings were extracted and tabulated. The methodological quality of studies was assessed using the 'Downs and Black Instrument', and the level of evidence for each study was determined using 'Centre for Evidence Based Medicine' criteria. Results: Eighteen studies were included in the review. From these the proportion returning to driving following a TBI and the time frames varied between $22.4 \%$ at 6 months post-injury to over $70 \%$ reported between 1 and 5 years post TBI. The variations in time that people took to return to driving reflected their severity of injury. Four themes were identified relating to the factors that influence return to driving - resource related, psychological factors, social barriers, associated health factors.

Conclusions: Information about rates of people returning to drive and the timing at which this occurs may be helpful for patient education and informing decision making by clinicians, patients and caregivers.
\end{abstract}

Correspondence: Janelle Griffin; janelle.griffin@health.qld.gov.au

\section{From Evidence to Practice: Supporting the Decision-Making Participation of Adults with Traumatic Brain Injury (TBI)}

\author{
Lucy Knox, ${ }^{1,2}$ Jacinta Douglas ${ }^{1,2}$ and Christine Bigby \\ ${ }^{1}$ Living with Disability Research Centre, La Trobe University, Melbourne, Australia \\ ${ }^{2}$ Summer Foundation, Victoria, Australia
}

$\mathcal{R}$ ackground and aims: The right to make decisions about one's own life

$\mathcal{B}$ is a fundamental tenet of the Convention on the Rights of Persons with Disabilities and a central aim of contemporary disability policy. However, there has been limited investigation of how people with TBI and those in their support network participate in making decisions. The aims of this research were: 1) to understand how adults with severe TBI and their supporters experience the decision-making process; and 2) from this evidence, to build a series of recommendations for clinical practice to maximise the decision-making participation of adults with TBI.

Method: Data was generated over two years, and included 42 in-depth interviews with adults with TBI and their decision-making supporters. Analysis drew on constructivist grounded theory methods. Through a process of 
constant comparison clear categories emerged and a substantive theory capturing the process of making decisions after brain injury was developed.

Results: The overarching finding reflected the relational nature of decisionmaking participation for adults with TBI. Three key constructs were evident: giving and receiving support, constructing the decision-making space, and conceptualising self. Practice strategies for health professionals to maximise the participation of adults with TBI in making decisions were clearly embedded within each construct.

Conclusions: This study is the first to simultaneously explore the experiences of adults with TBI and those around them in making decisions about life after injury. The results have yielded practice recommendations grounded in the lived experience of adults with TBI and those who support them.

Correspondence: Lucy Knox; L.Knox@latrobe.edu.au

\title{
Concurrent Session 5
}

\section{A Neuropsychological Analysis of Violent Offending}

\author{
David Andrews ${ }^{1}$ and Lisanne Jenkins ${ }^{2,3}$ \\ ${ }^{1}$ Melbourne Neuropsychiatry Centre, Department of Psychiatry, The University of Melbourne, Aus- \\ tralia \\ ${ }^{2}$ The School of Psychological Sciences, University of Melbourne, Australia \\ ${ }^{3}$ Department of Psychiatry, The University of Illinois at Chicago. USA
}

T mpulsive violent crime leads to incarceration in an overburdened prison system and an often leaves poorly supported victims. Therefore the identification of a brain-related individual predisposition towards violent and impulsive acts may help in the design of a treatment towards remediation and reduce recidivism. This review covers three areas of affective neuroscience in an analysis of the neuropsychology of emotional reactivity and violence. Several areas of research support a neuropsychological argument towards describing individuals who have a predisposition to violence associated with emotional dysregulation. Firstly, is the nature of the act itself and Libet's (1985), now confirmed findings, that action may be brain initiated prior to conscious intention and deliberation. Secondly, the visceral features of "flight and fight" and the authors' own work providing evidence that reduced levels of ventromedial prefrontal cortex (VMPFC) control result in excessive levels of emotional reactivity to threatening material (Jenkins et al, 2016). Thirdly, is the association between testosterone and violent offending and the influence of high levels and the interaction between the amygdala and the VMPFC. These factors are finally combined to provide a profile for the violent offender, which has been variously confirmed within prison population in persons who have a history of violence. Finally, there is an argument that certain treatments could be usefully tailored to persons with this neuropsychological profile.

Correspondence: David Andrews; andrewes@unimelb.edu.au 


\title{
Prefrontal Specialisation for Verbal and Nonverbal Idea Generation: A Neuropsychological Case Series
}

\author{
Gail A. Robinson \\ Queensland Neuropsychology Research Centre, School of Psychology, The University of Queensland, \\ Brisbane, Australia
}

\begin{abstract}
$B$ ackground and aims: Voluntary generation of ideas is a crucial executive ability for adaptive behaviour, associated with the prefrontal cortex. Idea generation involves both thinking and expression of ideas via words and sentences (verbal generation) or via gestures, drawing and problem solving (nonverbal generation). Verbal generation impairments have been associated with the left prefrontal cortex in patients with prefrontal lesions or cases of dynamic aphasia. The aim of this study was to investigate nonverbal generation and the associated neural correlates.

Methods: A series of eight patients with right prefrontal lesions and ten closely matched healthy controls were given baseline neuropsychological tests and a series of experimental nonverbal generation tests, including design fluency and motor movement selection, and verbal generation tasks (word, sentence, connected speech).

Results: The baseline neuropsychological tests indicated that the patient group performed broadly within the average range on tests of fluid intelligence, memory, language and visual perception. As expected, patients were impaired on some tests that tap executive abilities. For the experimental series, the right prefrontal patients were impaired on all non-verbal generation tasks. By contrast, they were largely intact on a series of verbal generation tasks.

Conclusions: This pattern suggests a role for the right prefrontal cortex in nonverbal generation. Moreover, this pattern of findings is a mirror contrast to that observed in patients with specific verbal generation deficits in the context of dynamic aphasia. Taken together, this supports specialisation within the prefrontal region for verbal and non-verbal generation.
\end{abstract}

Correspondence: Gail Robinson; g.robinson@psy.uq.edu.au

\section{Spousal Recollections of Early Manifestations of Primary Progressive Aphasia}

\author{
Margaret Pozzebon, ${ }^{1,2,3}$ Jacinta Douglas ${ }^{1,4}$ and David Ames ${ }^{3,4,5}$ \\ ${ }^{1}$ School of Allied Health, La Trobe University, Melbourne, Australia \\ ${ }^{2}$ Speech Pathology Department, Royal Melbourne Hospital, Melbourne, Australia \\ ${ }^{3}$ Cognitive Dementia and Memory Service, Royal Melbourne Hospital, Melbourne, Australia \\ ${ }^{4}$ Summer Foundation, Victoria, Australia \\ ${ }^{5}$ The National Ageing Research Institute and University of Melbourne, Melbourne, Australia \\ ${ }^{6}$ Department of Old Age Psychiatry, University of Melbourne, Melbourne, Australia
}

$\boldsymbol{R}$ ackground and aims: Although primary progressive aphasia (PPA) is 3 known to be characterised by progressive loss of language abilities, knowledge about the earliest symptoms is limited. This study sought to explore spousal recollections regarding the earliest manifestations of PPA and to compare the nature of the earliest perceived symptoms across the three PPA variants.

Method: In-depth interviews focusing on earliest symptoms of illness onset were conducted with 13 spouses whose partners were diagnosed with PPA. The data were collated, analysed and key themes identified and compared across the PPA variants.

Results: Spousal retrospective accounts indicated the 3 PPA variants (nonfluent, logopenic and semantic) had a signature profile announcing illness 
onset. The findings suggest the possibility that PPA initially presents as subtle changes in interpersonal-relational contexts for svPPA and nfvPPA rather than overt receptive and expressive language impairments. The initial symptoms for partners with nfvPPA were speech production and fluency issues. The nuances revealed through personal narratives illustrate the challenges associated with early identification, particularly as very early manifestations of PPA are unlikely to be easily captured in standardized clinical assessments, scales and questionnaires.

Conclusions: Understanding the nature of symptoms perceived in the earliest stages of PPA has potential to inform earlier and accurate diagnosis and interventions to assist those living with the illness.

Correspondence: Margaret Pozzebon; margaret.pozzebon@mh.org.au

\title{
Cognitive Fatigue in Multiple Sclerosis: Do Self-Reports of Fatigue Correspond with Actual Reductions in Cognitive Performance over a Single Testing Session?
}

\author{
Cynthia A. Honan, ${ }^{1}$ Caitlin Turner, ${ }^{1}$ Heather Francis, ${ }^{2}$ Ingrid van der $\mathrm{Mei}^{3}$ and \\ Edwin Lim $^{4}$ \\ ${ }^{1}$ Discipline of Psychology, School of Medicine, Faculty of Health, University of Tasmania, Launceston, \\ Australia \\ ${ }^{2}$ Department of Psychology, Faculty of Human Sciences, Macquarie University, Sydney, Australia \\ ${ }^{3}$ Menzies Institute for Medical Research, University of Tasmania, Hobart, Australia \\ ${ }^{4}$ Department of Biomedical Sciences, Faculty of Health Sciences, Macquarie University, Sydney, \\ Australia
}

$\boldsymbol{B}$ ackground and aims: Individuals with multiple sclerosis (MS) differentiate their experience of cognitive from physical fatigue. However, the concept of cognitive fatigue and how it is appraised is poorly understood. This study examines the relationship between self-reported fatigue and actual change in cognitive performance over a single testing session to further our understanding of MS-related cognitive fatigue.

Method: Thirty-one participants with MS and 30 matched healthy control participants completed the Modified Fatigue Impact Scale (mFIS) and were assessed twice on the Brief Repeatable Neuropsychological Battery (BRNB) and Conners Continuous Performance test (CPT) over a 2.5 hour testing session. A visual analogue scale for fatigue (VAS-F) was administered at baseline, and immediately following the first and second CPT administration.

Results: MS participants performed more poorly than healthy participants on most psychological tests, and performance over the session changed in a manner consistent with healthy controls on BRNB tasks. Whereas CPT performance declined over time in the MS participants, it improved in healthy participants. The MFIS had small-to moderate correlations with Conners performance. Further, whereas high correlations were detected between change in VAS-F scores and change in Conners index scores for the healthy control participants, there was negligible correlations for individuals with MS.

Conclusions: The results provide support for a temporal fatigue hypothesis in explaining MS-related fatigue. Individuals with MS also appear less able to accurately appraise changes in cognitive functioning, indicating that factors other than changes in levels of cognitive functioning are related to the experience of cognitive fatigue in MS.

Correspondence: Cynthia Honan; Cynthia.honan@utas.edu.au 


\title{
Concurrent Session 6
}

\section{Memory Impairment and Recovery in Post-Traumatic Amnesia}

\author{
Caroline Roberts, ${ }^{1,2}$ Gershon Spitz,, ${ }^{1,2}$ Matthew Mundy ${ }^{1}$ and Jennie Ponsford ${ }^{1,2}$ \\ ${ }^{7}$ Monash Institute of Cognitive and Clinical Neuroscience, School of Psychological Sciences, Monash \\ University, Melbourne, Australia \\ ${ }^{2}$ Monash-Epworth Rehabilitation Research Centre, Melbourne, Australia
}

\begin{abstract}
$B$ ackground and objectives: Observations of "shrinking retrograde amnesia' suggest that following traumatic brain injury (TBI) memories recover in order of remoteness. On prospective scales, items relating to well-established memories (e.g., date of birth) tend to recover earlier than more recent orientation items (e.g., age, year) and consistent new learning. However, there has been limited empirical research specifically examining retrograde amnesia. The present study aimed to determine whether a negative gradient in personal semantic (PS) memory (relative preservation of remote memories over more recent ones) exists in Post-Traumatic Amnesia (PTA).

Method: 20 patients with TBI and 20 healthy controls (HCs) were administered the Personal Semantic Schedule of the Autobiographical Memory Interview. The TBI group were assessed once during PTA (monitored using the Westmead Post-Traumatic Amnesia scale) and post-emergence. Repeated measures ANOVA was used to compare the gradient across lifetime periods in and out of PTA, and mixed model ANOVAs for comparison to the control group.

Results: PS memory was significantly impaired in PTA relative to out of PTA, and the TBI group performed significantly lower than controls at both time points. There was no interaction between group and lifetime period. Lower scores in PTA were associated with a greater number of days to emerge from PTA post interview.

Conclusions: These results suggest that there is a global impairment in PS memory across lifetime periods in PTA (no gradient). PS memory may be particularly sensitive to the diffuse nature of TBI and contribute to our understanding of PTA as an indicator of injury severity.
\end{abstract}

Correspondence: Caroline Roberts; caroline.roberts@monash.edu

\section{Agitation during Post Traumatic Amnesia: Characteristics, Predictors, and Impact on Therapy}

\author{
Adam McKay, ${ }^{1,2,3}$ Jessica Trevena-Peters ${ }^{1,3}$ and Jennie Ponsford ${ }^{1,3}$ \\ ${ }^{1}$ School of Psychological Sciences, Monash University, Melbourne, Australia \\ ${ }^{2}$ Department of Psychology, Epworth Rehabilitation, Melbourne, Australia \\ ${ }^{3}$ Monash-Epworth Rehabilitation Research Centre, Epworth Hospital, Melbourne Australia
}

$\boldsymbol{B}$ ackground and objectives: Agitation is a common feature of post trau3 matic amnesia (PTA) after TBI, however, reported frequencies vary across studies, assumptions that agitation interferes with rehabilitation participation have rarely been investigated, and relatively little is known about the relationship between agitation and cognitive impairments during PTA. The aims of this study were to examine: 1) the frequency and nature of agitation during PTA; 2) the impact of agitation on participation in therapy during PTA; and 3) the relationship between agitation levels and the cognitive impairments during PTA.

Method: Prospective assessment of agitation (Agitated Behavior Scale, $\mathrm{ABS}$ ), therapy participation (time in ADL retraining) and orientation/memory 
(Westmead PTA Scale) were conducted in consecutive patients who were admitted for inpatient rehabilitation during the PTA phase after severe TBI.

Results: Preliminary results based on 100 admissions show that agitation was observed in approximately $50 \%$ of participants (ABS score $>21$ ) in PTA, with disinhibited behaviours most common. Regression analyses found that agitation scores were not significantly associated with time spent in ADL retraining during PTA but they were associated with performance on the Westmead PTA Scale such that greater levels of disorientation and memory impairment predicted greater levels of agitation.

Conclusions: Agitated behaviour was common during PTA but it was not the presumed barrier to participation in active therapy during PTA. The observed association between agitation and concurrent cognitive impairments suggest that strategies to maximise orientation and memory may also help to minimise agitation.

Correspondence: Adam McKay; adam.mckay@monash.edu

\section{Evaluating Outcomes of Skills Retraining during Posttraumatic Amnesia Using Goal Attainment Scaling}

Jessica Trevena-Peters, ${ }^{1,2}$ Adam McKay, ${ }^{1,2}$ Rachel Suda, ${ }^{3}$ Zoe McMahon ${ }^{3}$ and Jennie Ponsford ${ }^{1,2}$

${ }^{1}$ Monash Institute of Cognitive and Clinical Neuroscience, School of Psychological Sciences, Monash University, Clayton VIC, Australia

${ }^{2}$ Monash Epworth Rehabilitation Research Centre, Richmond, VIC, Australia

${ }^{3}$ Epworth HealthCare, Richmond, VIC, Australia

$\boldsymbol{R}$ ackground and aims: Posttraumatic amnesia (PTA) following traumatic brain injury is characterised by confusion, agitation and anterograde amnesia. We evaluated the efficacy of activities of daily living (ADL) retraining during PTA. This paper will examine attainment of ADL goals using Goal Attainment Scaling (GAS) by patients receiving ADL retraining during PTA. Method: The treatment group included 39 patients with PTA $>7$ days. ADL retraining during PTA was manualised, followed errorless learning principles, and targeted personal care (bathing, grooming, dressing, self-feeding) and basic meal preparation. Following functional assessments, Occupational Therapists developed individualised goals using GAS framework with a 5-point outcome scale $(-2$ to +2$)$. Goals were scored at PTA emergence as below expectation $(-2,-1)$, at expectation $(0)$, or above expectation $(+1,+2)$.

Results: Thirty-four participants had GAS completed, with $5(12.82 \%)$ deemed independent following assessment. Eighty-nine goals were set for the 34 participants. GAS outcomes at PTA emergence were 6 below expectation (6.74\%), 14 at expectation (15.73\%), and 69 above expectation (77.53\%). GAS outcomes were relatively consistent across domains of intervention. Sixty-eight personal care goals were developed with outcomes of 6 below $(8.82 \%), 8$ at $(11.76 \%)$ and 54 above $(79.41 \%)$ expectation. Seventeen participants had basic meal preparation goals; 4 performed at $(23.53 \%)$, and 13 above $(76.47 \%)$ expectation. Four participants had goals extending beyond an achieved goal, 2 performed at expectation, and 2 above.

Conclusion: Results demonstrate that individuals in PTA have potential to make substantial gains in response to ADL retraining across domains of personal care and basic meal preparation.

Correspondence: Jessica Trevena-Peters; jessica.trevena-peters@monash. edu 


\title{
Concurrent Session 7 - How to Session 2
}

\section{How to Work within the National Disability Insurance Scheme with People with Acquired Brain Injury}

\author{
Libby Callaway, ${ }^{1,2}$ Jan Mackey, ${ }^{3}$ Sue Sloan ${ }^{4}$ and Natasha Lannin ${ }^{5,6}$ \\ ${ }^{1}$ Department of Occupational Therapy, School of Primary and Allied Health Care, Monash University, \\ Frankston, Australia \\ ${ }^{2}$ Neuroskills Pty Ltd, Sandringham, Australia \\ ${ }^{3}$ Applied Communication Skills, Clifton Hill, Australia \\ ${ }^{4}$ Osborn Sloan and Associates, Kew, Australia \\ ${ }^{5}$ Occupational Therapy Department, Faculty of Health Sciences, La Trobe University, Melbourne, \\ Australia \\ ${ }^{6}$ Alfred Health
}

\begin{abstract}
C ynopsis of session: The introduction of Australia's National Disability $\mathcal{N}$ Insurance Scheme (NDIS), due for full implementation by 2019, radically changes the way people with disability plan for and receive support. For allied health clinicians working with people with ABI, it is necessary to understand the practical application of this major policy reform to work effectively within the Scheme.

Delivered by an interdisciplinary group experienced in both policy and clinical practice application of the NDIS, the session will provide an overview of how to maintain best practice community ABI rehabilitation approaches within an NDIS context. It will include:
\end{abstract}

- An overview of relevant NDIS policies and frameworks, as well as pricing guidelines;

- Useful preparation and planning tools for NDIS participants and their support networks;

- Strategies to facilitate or enable people who experience cognitivecommunication difficulties to effectively participate in the NDIS planning process;

- Clinical case scenarios, with a focus on social communication and positive behavioural support to effectively influence long term participation outcomes.

\section{Concurrent Session 8}

\section{Which Clinician Competencies are Important for Running Groups in Neurorehabilitation?}

Dana Wong

School of Psychological Sciences \& Monash Institute of Cognitive and Clinical Neurosciences, Monash University, Melbourne, Australia

Monash-Epworth Rehabilitation Research Centre, Melbourne, Australia

\begin{abstract}
$B$ ackground and aims: Group-based neurorehabilitation programs can be effective and cost-efficient. However, little is known about how manualised programs are implemented in practice, or the competencies necessary for facilitators to run effective groups. Training competent clinicians is essential for clinical translation. This study aimed to develop a checklist of group facilitation competencies for use in research, training, and clinical settings.
\end{abstract}


Method: The Delphi method for obtaining expert consensus was adopted to identify relevant competencies. Experts were clinicians or researchers with expertise in running groups. The importance and clarity of 17 checklist items were rated by 15 experts. There were four item categories: Facilitating focused group discussion, Communication skills, Interpersonal style, and Session structure. The Delphi criterion of $>80 \%$ of experts endorsing the two highest ratings determined item inclusion, using an iterative review process. Qualitative feedback was sought for each item, and experts were encouraged to suggest additional items.

Results: After the first round of review, two items were removed after failing the consensus criterion, the wording of five items was revised, and two new items were added. In the second review round, all items met consensus criterion, and two further wording adjustments were made. Agreement tended to be higher for items in the Facilitating focused group discussion and Communication skills categories.

Conclusions: A high level of expert consensus was reached on a new 17item group facilitation competency checklist. After establishing inter-rater reliability, the checklist can be used to evaluate clinician competencies in group facilitation, and explore relationships with participant outcomes.

Correspondence: Dana Wong; dana.wong@monash.edu

\title{
It Takes 2 to Tango: The Therapeutic Alliance in Community Brain Injury Rehabilitation
}

\author{
Liz M. Williams ${ }^{1,2}$ and Jacinta M. Douglas ${ }^{1,3}$ \\ ${ }^{1}$ Living with Disability Research Centre, School of Allied Health, La Trobe University, Melbourne, \\ Victoria, Australia \\ ${ }^{2}$ Brain Injury Rehabilitation Community and Home BIRCH, South Australian Brain Injury Rehabilitation \\ Service, Hampstead Rehabilitation Centre, Adelaide, Australia \\ ${ }^{3}$ Summer Foundation, Box Hill, Victoria, Australia
}

$\mathcal{R}$ ackground and aims: A positive therapeutic (or working) alliance has been associated with better outcomes in psychotherapeutic literature and recently in traumatic brain injury (TBI) rehabilitation literature. The aim of this study was to understand the perspectives of adults with TBI and their significant others regarding a) where the therapeutic alliance sits in the process of rehabilitation, and b) what helps and doesn't help the alliance.

Method: A constructivist, qualitative methodology using grounded theory analysis techniques was applied. Three participant pairs (an adult with TBI and significant other) who had completed a community rehabilitation program took part in the study. Data collection involved in-depth interviews with each individual participant. Interviews were transcribed verbatim and progressively analysed using a process of constant comparison.

Results: A framework illustrating participants' experience of a therapeutic alliance was generated, comprising three interconnected themes: being treated like an individual, working together, and feeling connected. All participants viewed being able to work together as important in their experience of community rehabilitation and described features that help and hinder the alliance. Conclusion: Therapeutic alliances make an important contribution to the rehabilitation experience of individuals with TBI and those close to them. Further research is needed to explore the nature of the alliance from the perspective of rehabilitation professionals and to understand how it affects outcomes in brain injury rehabilitation.

Correspondence: Liz Williams; liz.williams@sa.gov.au 


\title{
Rehabilitation Groups - What is Unique about Groups in Traumatic Brain Injury Rehabilitation?
}

\author{
Freyr Patterson, ${ }^{1}$ Jennifer Fleming ${ }^{1,2}$ and Emmah Doig ${ }^{1}$ \\ ${ }^{1}$ School of Health and Rehabilitation Sciences, The University of Queensland, Brisbane Australia \\ ${ }^{2}$ Occupational Therapy Department, Princess Alexandra Hospital, Brisbane, Australia
}

\begin{abstract}
$B$ ackground and objectives: Groups are commonly used in occupational therapy and rehabilitation practice. Opportunities for peer support and maximising intensity of rehabilitation, and cost-effectiveness are some of the benefits of rehabilitation groups. Currently there is limited evidence to guide clinicians in the delivery of groups to patients with traumatic brain injury (TBI). The aims of this study were to explore the perspectives of occupational therapists about the benefits, limitations and processes of groups in rehabilitation, and to identify aspects of rehabilitation groups that are unique to TBI.

Method: This study was guided by a phenomenological approach to explore the experience of conducting rehabilitation groups with clients with TBI. Data were collected through a series of focus groups with occupational therapists $(N=26)$ working in brain injury, spinal cord injury and geriatric rehabilitation settings at a major metropolitan hospital. Framework analysis was utilised to analyse the data.

Results: Clinicians agreed cognitive impairments and challenging behaviours, which are common following TBI, impacted on group processes. Group and behaviour management skills, and facilitator confidence emerged strongly as particularly important when facilitating TBI groups. Forward planning, including processes to achieve a 'good fit' of participants and to ensure groups addressed individual patient goals was highlighted as especially important in TBI group rehabilitation.

Conclusions: Overall, clinicians reported that groups contributed positively to rehabilitation. Thorough planning processes and facilitator skills, particularly in the area of behaviour management and tailoring group content to enable participation of people with cognitive impairment was emphasised as paramount in TBI group rehabilitation.
\end{abstract}

Correspondence: Freyr Patterson; freyr.patterson@uqconnect.edu.au

\section{Narrative Storytelling Following Acquired Brain Injury: Sharing My Story to Help Others.}

\author{
Kate D'Cruz, ${ }^{1}$ Jacinta Douglas ${ }^{1,2}$ and Tanya Serry ${ }^{1}$ \\ ${ }^{1}$ School of Allied Health, La Trobe University, Melbourne, Australia \\ ${ }^{2}$ The Summer Foundation, Melbourne, Australia
}

ackground and aims: Storytelling is an everyday human experience.
Following trauma or disruption to our life-narrative, sharing personal
stories helps us regain coherence by integrating aspects of the past narrative
within the context of the new. Personal stories are also used to raise public
awareness and to help others appreciate the impact of social and health issues
on a person's life. The aim of this grounded theory study is to better understand
the experience and impact of narrative storytelling from the perspective of four
groups: 1) adult storytellers with acquired brain injury (ABI); 2) storytelling
facilitators; 3) close others of storytellers; and 4) the audience who have
witnessed the stories. This presentation will focus upon the experiences of the
storyteller participants.
Method: In-depth interviews were used to explore the experiences of eight
adults with severe ABI who had participated in storytelling workshops to 
produce a personal narrative. All participants have significant cognitive and/or communication impairments, including some with severe dysarthria and use of assisted augmentative communication (AAC) devices. Interview transcripts were analysed using constructivist grounded theory methods.

Results: Analysis revealed several themes: sharing my story to help others, re-visiting memories during storytelling, releasing emotions through storytelling, and the missed opportunity of re-visiting stories. These themes not only captured insights into the process of storytelling itself but also revealed substantial implications for goal setting and intervention in rehabilitation.

Conclusions: These findings provide further evidence to support the therapeutic potential of narrative storytelling in the lives of adults with ABI.

Correspondence: Kate D’Cruz; k.dcruz@latrobe.edu.au

\title{
Do Rehabilitation Goals Reflect Important Life Roles? Barriers and Enablers to Articulating Meaningful Goals Following an Acquired Brain Injury
}

\author{
Danielle Sansonetti, ${ }^{1,2}$ Rebecca Nicks, ${ }^{2}$ Carolyn Unsworth ${ }^{3}$ and Natasha Lannin ${ }^{2,4}$ \\ ${ }^{1}$ Acquired Brain Injury Rehabilitation Centre, Alfred Health, Caulfield, Australia \\ ${ }^{2}$ Occupational Therapy Department, Alfred Health, Melbourne, Australia \\ ${ }^{3}$ School of Health, Medical and Applied Sciences, Central Queensland University, Melbourne \\ ${ }^{4}$ Discipline of Occupational Therapy, School of Allied Health, College of Science, Health and Engi- \\ neering, La Trobe University, Bundoora, Australia
}

$\boldsymbol{B}$ ackground and objectives: Articulating life roles within a patient3 directed goal framework is both important and challenging for clinicians working with clients with severe acquired brain injury. Evidence recommends the use of patient-directed goal setting, however little is known about how to best integrate life roles discussions into the goal-setting process. This study aims to identify barriers and enablers to embedding role discussions within a patient-directed goal framework in a state-wide acquired brain injury rehabilitation service.

Method: A mixed method study was completed. Focus groups with a total of 6 clinicians and 6 patients and carers were conducted to identify barriers and enablers to articulating goals associated with important life roles within an inpatient rehabilitation setting. Data were analysed thematically. A file audit was completed to further investigate the alignment of rehabilitation goals and life roles that were documented across the inpatient rehabilitation admission. Results: Focus group interviews with clinicians indicated that patient factors, time, environment, and organisational expectations influenced their ability to align goals with patient roles. Patient and carer interviews suggested the need for more regular review of goals throughout rehabilitation to make this process more meaningful. File audit data demonstrated variable alignment of goals and life roles. Barriers and enablers to aligning goals with important life roles will be further discussed, together with practice implications.

Conclusion: Translating patient and team goals into a life role framework empowers both patients and families following severe acquired brain injury. Achieving this ideal can be challenging.

Correspondence: Danielle Sansonetti; D.Sansonetti@alfred.org.au 
Plenary 2

\title{
The Long and Winding Road - (Social) Cognition and Sense of Identity in Dementia
}

Olivier Piguet

Brain and Mind Centre, the University of Sydney, Sydney, Australia

School of Psychology, the University of Sydney, Sydney, Australia

ARC Centre of Excellence in Cognition and its Disorders, Sydney, Australia

ocial/emotion cognition plays a crucial role in how we apprehend the $\int$ world: it helps define our interactions with other individuals and also contribute to cognitive performance. Disturbance of social/emotional cognition is a common feature of a number of dementia syndromes. In this keynote address, I will discuss the main facets of social/emotional processing disturbance in dementia, their impact on other aspects of cognition (e.g., memory, executive function), and what these deficits tell us about who we are as humans.

Correspondence: Olivier Piguet; olivier.piguet@sydney.edu.au

\section{SATURDAY 3rd JUNE}

\section{Plenary 3}

\section{Anger Self-Management Training in Chronic Severe Traumatic Brain Injury: Lessons Learned from a Randomized Controlled Trial}

Tessa Hart

Moss Rehabilitation Research Institute, Elkins Park, PA, USA

\begin{abstract}
D roblematic anger and irritability affect up to $1 / 3$ of survivors of traumatic brain injury (TBI) across the spectrum of severity. Unlike some other neuropsychiatric symptoms, anger may not remit over time but may be exacerbated by ongoing frustrations with cognitive deficits, loss of independence, and a restricted sphere of activity and social relationships. Treatment options are few, and anger may exclude survivors from rehabilitation programs. In this address, I will describe the background, rationale, and findings from a randomized controlled trial testing a specialized treatment protocol, Anger Self-Management Training (ASMT), which was designed specifically for people with chronic TBI, problematic anger, and significant cognitive deficits. The ASMT was tested against a structurally equivalent intervention designed for the trial, which emphasized education, ventilation of feelings, and psychological support. This presentation will include not only the results of the trial, which was completed with 90 participants, but lessons learned about the important components of clinical trials including equipoise, masking, and the selection or design of control conditions. I will also discuss the specific techniques and ideas within the ASMT protocol that participants found to be most effective in managing their anger in everyday life.
\end{abstract}

Correspondence: Tessa Hart: thart@einstein.edu 


\title{
From Research to Practice - Knowledge Translation in Neurorehabilitation
}

William Levack

Rehabilitation Teaching Research Unit, University of Otago, Wellington, New Zealand

n 22 October 2016, Cochrane endorsed a new Field within its organization: Cochrane Rehabilitation. The primary purpose of this Field is to connect people involved in generating research evidence in rehabilitation to those who apply this evidence in clinical practice. This relationship is intended to be bi-directional, with Cochrane Rehabilitation providing both a pathway for relevant work within Cochrane to be disseminated to rehabilitation stakeholders and providing a forum by which rehabilitation stakeholders can inform and influence the work of Cochrane. While systematic reviews of randomized controlled trials often provide excellent evidence to direct clinical practice (indeed systematic reviews of systematic reviews, called Cochrane Overviews, are an area of increasing activity) there is growing awareness of the limitations of these approaches in the context of clinical rehabilitation. Many areas of work in neurorehabilitation are difficult to study with randomized controlled trial methods due to factors such as the heterogeneity of patient populations and the need for individualization of patient interventions. Drawing on experiences of undertaking clinical trials and systematic reviews in the context of goal setting in rehabilitation, this presentation will provide a contemporary view on knowledge translation in neurorehabilitation to inform clinical practice.

\section{Concurrent Session 9}

\section{The Efficacy of Environmental Cues to Reduce Length of Post-Traumatic Amnesia after Brain Injury: A Pilot Randomised Controlled Trial}

\author{
Natasha A. Lannin, ${ }^{1,2}$ Carolyn Unsworth, ${ }^{1,3}$ Megan Coulter, ${ }^{2}$ Russell Gruen, ${ }^{4}$ Julia \\ Schmidt, ${ }^{1,5}$ Claire Galea ${ }^{6}$ and Tamara Ownsworth ${ }^{7}$ \\ ${ }^{7}$ La Trobe University, Melbourne, Victoria, Australia \\ ${ }^{2}$ Occupational Therapy Department, Alfred Health, Victoria, Australia \\ ${ }^{3}$ Central Queensland University, Queensland, Australia \\ ${ }^{4}$ Nanyang Institute of Technology in Health and Medicine, Nanyang Technological University, Singa- \\ pore \\ ${ }^{5}$ University of British Columbia, Vancouver, Canada \\ ${ }^{6}$ Cerebral Palsy Alliance, The University of Sydney \\ ${ }^{7}$ Menzies Health Institute Queensland, Griffiths University, Queensland, Australia
}

ntroduction: Patients in post-traumatic amnesia (PTA) after traumatic brain injury (TBI) experience disorientation to location, time and identity of others. Extended length of time in PTA has significant negative effects on engagement in rehabilitation and long-term outcome. Reorientation programs are thus an important component of neurotrauma treatment. However, it is essential to determine the most effective acute hospital environmental reorientation program to shorten the time to emergence from PTA.

Method: Randomised controlled trial with concealed allocation and intentionto-treat analysis. $N=40 \mathrm{TBI}$ participants were recruited on admission to acute care. The control group received usual care (inconsistent verbal orientation); the intervention group received a standardised environmental reorientation program aimed to improve orientation to person (signage, photographs and familiar items), place (signage and cueing) and time (calendar clock and cueing 
to environment). The primary outcome of time to emergence from PTA was measured by the Westmead PTA scale administered daily.

Results: There were no adverse responses to the environmental orientation program. While differences between groups in time to emerge from PTA were not statistically significant, the average time to emergence was shorter for those who received the standardised environmental reorientation program (11.0 days (95\% CI 6.8-15.2) versus 23.0 days (95\% CI 10.8-35.2)). This positive trend in favour of the intervention group suggests that future research include a larger sample to ensure adequate power.

Conclusion: Our environmental orientation program received good acceptance and promising indications of efficacy. However, the findings are limited by the small sample size and are not yet conclusive.

Correspondence: Natasha Lannin; n.lannin@ latrobe.edu.au

\title{
An Investigation of White Matter Integrity and Attention Deficits Following Traumatic Brain Injury
}

\author{
Jacqueline Owens, ${ }^{1,2,3}$ Gershon Spitz,, ${ }^{1,2} 3$ Jennie Ponsford, ${ }^{1,2,3}$ \\ Alicia Dymowski, ${ }^{1,2,3}$ and Catherine Willmott ${ }^{1,2,3}$ \\ ${ }^{1}$ School of Psychological Sciences, Monash University, Melbourne, Australia \\ ${ }^{2}$ Monash-Epworth Rehabilitation Research Centre, Epworth HealthCare, Melbourne, Australia \\ ${ }^{3}$ Monash Institute of Cognitive and Clinical Neurosciences, Monash University, Melbourne, Australia
}

$B$ ackground and aims: Attention and working memory deficits are prominent following moderate-severe traumatic brain injury (TBI), however the mechanisms underpinning these deficits are poorly understood. This study aimed to investigate the association between white matter tracts and multiple aspects of attention and working memory deficits following moderate-severe TBI.

Method: Neuropsychological measures of attention and working memory were administered to 20 participants with complicated mild-severe TBI (post traumatic amnesia (PTA) $\mathrm{M}=40.05 \pm 37.10$ days, median time since injury 10.48 months, range 3.72-87.49) and 20 control participants. Tract-based spatial statistics were used to assess fractional anisotropy (FA) and mean diffusivity (MD) of white matter tracts for 15 TBI participants and 20 controls. Results: When compared to controls, participants with TBI were found to have lower FA $(p<.001)$ and higher MD $(p<.001)$ values in the majority of white matter tracts. TBI participants were also slower to complete tasks including Trail Making Task, Hayling, Computerised Selective Attention Task, $n$-back, and Symbol Digit Modalities Test $(p<.001)$, when compared to controls. When controlling for age and estimated pre-morbid intelligence, slowed information processing speed following TBI was found to be associated with FA values in the corpus callosum, superior longitudinal fasciculus, cingulum, inferior fronto-occipital fasciculi, corona radiata, and cerebral white matter. Conclusion: The results highlight the widespread damage associated with TBI, as well as the impact of these alterations on information processing speed.

Correspondence: Jacqueline Anne Owens; Jacqueline.Owens@monash.edu 


\title{
Head Impact Biomechanics in Non-Concussed, Amateur Australian Football Players and Association with SCAT3
}

\author{
Catherine Willmott, ${ }^{1,2}$ Andrew S. Mclntosh,, ${ }^{3,4}$ Teresa Howard, ${ }^{5,10}$ Biswadev \\ Mitra, ${ }^{5,6,7}$ Bleydy Dimech-Betancourt, ${ }^{1,2}$ Jarrod Donovan, ${ }^{5,7}$ and Jeffrey V. \\ Rosenfeld $8,9,10,11$ \\ ${ }^{1}$ School of Psychological Sciences, Monash Institute of Cognitive and Clinical Neurosciences, \\ Monash University \\ ${ }^{2}$ Monash-Epworth Rehabilitation Research Centre, Melbourne, Victoria \\ ${ }^{3}$ Australian Centre for Research into Injury in Sport and its Prevention, Federation University \\ ${ }^{4}$ Monash Injury Research Institute, Monash University \\ ${ }^{5}$ National Trauma Research Institute, Melbourne, Victoria \\ ${ }^{6}$ Department of Epidemiology \& Preventive Medicine, Monash University \\ ${ }^{7}$ Emergency \& Trauma Centre, The Alfred Hospital, Melbourne, Victoria \\ ${ }^{8}$ Monash Institute of Medical Engineering, Monash University \\ ${ }^{9}$ Department of Neurosurgery, The Alfred Hospital, Melbourne, Victoria \\ ${ }^{10}$ Department of Surgery, Monash University \\ ${ }^{11}$ Department of Surgery, F. Edward Hébert School of Medicine, Uniformed Services University of \\ The Health Sciences (USUHS), Bethesda, Maryland, USA
}

$\boldsymbol{R}$ ackground and aims: To quantify head impacts in non-concussed am$B$ ateur Australian footballers and association with post-game changes in symptom report, cognitive and physical function in a prospective cohort study. Methods: Male $(n=46)$ and female $(n=22)$ footballers. Peak linear acceleration (PLA) of the head ( $>10 \mathrm{~g})$ measured by wearable impact sensor X2 Biosystems X-Patch. SCAT3 administered at baseline (B) and post-game (PG).

Results: $N=2088$ impacts $>10 \mathrm{~g}$, mean PLA $=14.41 \mathrm{~g}$ (10.54). Mean cumulative PLA $=560 g$ (576.67). No significant difference in mean PLA $(g)$ across gender $(U=288.00, z=1.24, p=.217)$, however, males sustained more impacts $(U=214.00, z=-2.46, p=.013)$. A greater number $(p=$ $.006)$ and severity $(p=.001)$ of symptoms were reported PG. Between group comparisons for B-PG SCAT3 change scores did not identify any significant differences between the Impact and No Impact groups $(p>.05)$. No significant association between number of impacts or mean PLA, and SCAT3 change scores $(p>.05$ for all) was identified, other than females with higher mean PLA made more balance errors post-game $\left(r_{\mathrm{s}}=-0.48, p=.04\right)$.

Conclusion: This investigation within Australian football provides preliminary evidence for comparison of head impacts across other codes (e.g. NFL, rugby union). Increase in symptom severity was independent of impact. Males sustained more impacts, however greater PLA was associated with reduced balance for females. Further evaluation of gender effects in impact biomechanics is required.

Correspondence: Catherine Willmott; catherine.willmott@monash.edu

\section{Deficits in the Eye Movement System Following Traumatic Brain Injury (TBI)}

Suzane Vassallo ${ }^{1}$ and Jacinta Douglas ${ }^{1,2}$

${ }^{1}$ Eye Movement Laboratory, School of Allied Health, La Trobe University, Melbourne, Australia

${ }^{2}$ Summer Foundation, Melbourne, Australia

$\boldsymbol{B}$ ackground and aims: The cortical areas controlling eye movements are vast and include frontal, striatal, parietal, occipital, cerebellar and brainstem regions. These areas and their interneural connections are affected following TBI, with individuals often demonstrating difficulty in eye 
movement control. The aim of this study was to develop a clinical summary of ocular motor deficits and their functional implications following TBI.

Method: A systematic literature review was undertaken. The search excluded blast-related injury and was limited to moderate to severe TBI. Eye movement control parameters included: latency, accuracy, velocity and errors of inhibition.

Results: The saccadic eye movement system has been the most frequently investigated visual system in TBI. Findings showed increased reaction time to saccade initiation, reduced accuracy, difficulty with predicting target movement and reduction in ability to generate volitional 'self-paced' saccades. Lack of inhibition of unwanted saccadic movement was also recorded, demonstrated by increased errors on antisaccade testing and saccadic intrusions during fixation. Smooth pursuit eye movements showed reduced speed and accuracy.

Conclusions: Deficits in eye movement control have important functional implications. In TBI deficits are exemplified by a lack of planning, difficulty orienting attention and inability to inhibit unwanted movement. Ocular motor findings like these may co-exist with similar behaviours demonstrated in social interactions. Unsteady fixation can easily disrupt reading performance, leading to the appearance of words moving on a page. Increased reaction time to execute an eye movement can increase time taken to complete visual tasks, with subsequent fatigue on the part of the individual.

Correspondence: Suzane Vassallo; S.Vassallo@latrobe.edu.au

\section{Exploring Eye Gaze Technology to Unlock Potential for Young People Living with Acquired Brain Injury}

Tanya Cavlovic ${ }^{1}$

${ }^{1}$ Disability Services Marangaroo, Brightwater Care Group, Perth, Australia

$\boldsymbol{R}$ ackground and aims: Assessing cognitive and communicative abilities of people with complex disability following severe acquired brain injury (ABI) can present significant challenges for health professionals. Failure to accurately assess and recognize potential will impact on the individual's participation in everyday life. Individuals are at risk of misdiagnosis, sub therapeutic services and potentially, withdrawal of services. Eye Gaze Technology is well utilised within the disability and assistive technology sectors but its use for assessment and as a recreational tool for young adults with complex disability is not mainstream. This study explores how the technology can support practical assessment, and provide meaningful recreation.

Method: Over 5 months, 10 clients received weekly sessions with trained staff using the Tobii Dynavox I12+ with specialist Eye Gaze software. Case study methodology was employed to investigate the potential of clients with differing needs and abilities. Client progress was measured using software analysis tools, including heat maps.

Results: Clinical assumptions were challenged, participant engagement was higher than expected and many demonstrated enjoyment and ongoing skill development. Outcomes have influenced care, therapy, and goal setting.

Conclusions: Eye Gaze was a useful tool that supported clinical assessment of people with complex disability following ABI. It has been embraced as a stimulating leisure activity that provides enjoyment as well as opportunity for further learning and functional gain.

Correspondence: Tanya Cavlovic; Tanya.Cavlovic@brightwatergroup.com 


\section{Development of a VR-based PM Task for Individuals with Brain Injury}

Christy Hogan, ${ }^{1}$ Petrea Cornwell, ${ }^{1,4}$ Jennifer Fleming ${ }^{2,3}$ and David Shum ${ }^{1}$

${ }^{7}$ School of Applied Psychology and Menzies Health Institute Queensland, Griffith University, Brisbane, Australia

${ }^{2}$ School of Health and Rehabilitation Sciences, The University of Queensland, Brisbane, Australia

${ }^{3}$ Occupational Therapy Department, Princess Alexandra Hospital, Brisbane, Australia

${ }^{4}$ The Prince Charles Hospital Metro North Hospital and Health Service, Brisbane, Australia

$\boldsymbol{B}$ ackground and aims: Prospective Memory (PM) impairment is common $\mathcal{B}$ after brain injury and affects every day functioning and independence. However, few assessment tools have been developed to measure the nature and extent of this problem. This study aimed to use a virtual reality (VR) platform to develop a PM task (the Virtual Reality Prospective Memory Shopping Task [VRPMST]) that is ecologically relevant and analytic.

Method: The study had 15 participants (six individuals with stroke and nine healthy controls). All of them completed the VRPMST, a self-report User Friendliness Scale - VRPMST, and a number of other neuropsychological tests. Time- vs. event-based PM as well as focal vs. non-focal PM cues were included in the VRPMST to understand the nature of PM impairment after brain injury.

Results: Results indicate that the VRPMST is user friendly. Participants agreed/strongly agreed that the task was similar to everyday activities ( stroke $=100 \%$; control $=78 \%)$; interesting $($ stroke $=83 \%$; control $=78 \%)$; had clear and easily understandable instructions (stroke $=83 \%$; control $=89 \%$ ); and would recommend it to others ( stroke $=100 \%$; control $=78 \%$ ). As expected, the two groups of participants showed similar performance on the ongoing task but the control group performed better than the stroke group on the PM tasks. Moreover, the time-based PM task was found to be more difficult than its event-based counterpart. Finally, performance in the focal condition was found to be better than that in the non focal condition.

Conclusions: Findings of the study provide support for the sensitivity and utility of the VRPMST in individuals with brain injury.

Correspondence: Christy Hogan; christy.hogan@griffithuni.edu.au

\section{Concurrent Session 10}

\section{Merri Community Aphasia Group: A Phase I Clinical Trial for Chronic Post-Stroke Aphasia}

Miranda Rose, ${ }^{1}$ Danica Dalton, ${ }^{2}$ Michelle Attard, ${ }^{1}$ Marcella Carragher ${ }^{1}$ and Lucette Lanyon ${ }^{1}$

${ }^{1}$ La Trobe University, Melbourne, Australia

${ }^{2}$ Merri Health, Melbourne, Australia

$\boldsymbol{R}$ ackground and objectives: Research investigating the specific mechaB nisms and processes within successful community aphasia groups (CAGs) is limited. We aimed to investigate the impacts of the Merri CAG for people with chronic aphasia (PWA) on each of the WHO ICF domains, along with carer (relative) burden.

Method: We used a Phase I (proof-of-concept) observational design. The group involved seven PWA and was facilitated by a speech pathologist with support from an allied health assistant. The program comprised of 12, 90- 
minute structured group sessions and took place at Merri community health centre. A range of outcome measures covering communication skills and confidence, mood, social networks, and carer burden were taken at pre-treatment, immediately post-treatment, and at 4-week follow-up.

Results: Group-level statistical analyses indicate PWA made clinically significant post-treatment gains across three domains (impairment, contextual factors, quality of life), with maintenance for impairment.

Individual results indicate six of the seven participants made clinically significant post-treatment gains in at least one domain-with four participants making gains in three to four. Most participants maintained gains for some or all domains. Relatives made no group-level change across time; individually, two of the seven made post-treatment gains with one evidencing maintenance. Analysis of PWA participation and relative skill in conversation are underway. Conclusions: In the short term, the Merri CAG was efficacious in improving PWA's outcomes on a broad level, with encouraging findings for maintenance overall. Findings will be discussed in relation to implications for CAG research and service delivery in chronic aphasia rehabilitation.

Correspondence: Miranda Rose; M.Rose@ latrobe.edu.au

\title{
A Detailed Program Model for a Multidisciplinary Community Aphasia Group: Results from a Phase I Clinical Trial
}

\author{
Michelle Attard, ${ }^{1}$ Leanne Togher, ${ }^{2}$ Yasmine Loupis, ${ }^{3}$ and Miranda Rose ${ }^{1}$ \\ ${ }^{1}$ La Trobe University, Melbourne, Australia \\ ${ }^{2}$ The University of Sydney, Sydney, Australia \\ ${ }^{3}$ St Vincent's Hospital, Sydney, Australia
}

$\boldsymbol{B}$ ackground and objectives: Research investigating the specific mechanisms and processes within successful community aphasia groups (CAGs) is limited. We tested the efficacy of a speech pathologist- and social worker-led CAG for four people with aphasia (PWA) and their spouses.

Method: We investigated the efficacy of a 2-hour $\times 12$-week speech pathologist- and social worker-led CAG protocol. The Therapeutic Factors Inventory (TFI) was administered at regular intervals during groups to measure perceived group experience. Outcome measures (pre, post, and 1-month follow-up) spanned the WHO ICF domains. We conducted case-based data analyses.

Results: Presence of therapeutic factors (TFI) was medium-high at Week 3 and either maintained or showed clinically significant increases at Week 12 for all PWA and spouses. Immediately post group, individual case analyses indicate clinically significant and/or meaningful gains for all four PWA on at least one WHO IFC domain (three showed gains across more than two to four domains). At follow up, the majority of gains were maintained, two PWA made initial gains, and one showed a decrease for the Personal domain of the ALA compared with pre-treatment. Descriptive analysis of social networks indicated an increase in the number of individuals for all participants across time.

For the spouses, one of the four evidenced reduced carer burden following the group, with maintenance at follow up.

Conclusions: This CAG resulted in a range of positive outcomes both in the short and longer term, predominantly for PWA. Implications for CAG research and service delivery in chronic aphasia rehabilitation will be discussed.

Correspondence: Miranda Rose; m.rose@latrobe.edu.au 


\title{
A Meta-Synthesis of the Qualitative and Quantitative Evidence for Community Aphasia Group Practice
}

Lucette Lanyon, ${ }^{1}$ Michelle Attard, ${ }^{1}$ Leanne Togher, ${ }^{2}$ Linda Worrall ${ }^{3}$ and Miranda Rose $^{1}$

${ }^{1}$ La Trobe University, Melbourne, Australia

${ }^{2}$ The University of Sydney, Sydney, Australia

${ }^{3}$ The University of Queensland, Brisbane, Australia

\begin{abstract}
$B$ ackground and aims: There is growing interest in the support and development potential of community aphasia groups (CAGs) for people living with chronic aphasia (PWA). Groups provide long-term and financially viable service options, addressing communication and psychosocial goals. However, up until recently no review of CAGs was available. We synthesised the evidence concerning the impacts of CAGs for PWA and significant others (SOs).

Method: A systematic review of the empirical quantitative evidence was completed in 2013. We used the International Classification of Functioning, Disability and Health (ICF) framework to categorise the outcomes of each study. We completed a second, narrative review in 2015 to investigate the consumer (PWA and SO) experience within the qualitative literature. We chose the theoretical construct of psychological well-being to frame and synthesise the results. Both reviews evaluated studies for level of evidence and methodological quality. More recent additions to the evidence have also been synthesised. Results: We identified 29 and 11 studies in the quantitative and qualitative reviews, respectively. CAGs can improve specific linguistic processes, social networks, and community access. However, there is limited evidence demonstrating improvement in functional communication. CAGs can also positively contribute to psychological wellbeing (meaningful relationships, purpose in life, environmental mastery, autonomy, personal growth, and self-acceptance) in PWA and SOs.

Conclusion: CAGs demonstrate potential across a range of areas. Existing research reflects limited consistency in aims, treatment programs, and outcome measures. Future studies must consider involving both quantitative and qualitative measures to effectively capture the range of potential outcomes of CAGs.
\end{abstract}

Correspondence: Lucette Lanyon; 1.lanyon@latrobe.edu.au

\section{The Experience of Close Personal Relationships from the Perspective of People with Aphasia: Thematic Analysis of the Literature}

\author{
Amy Ford, ${ }^{1,2}$ Jacinta Douglas, ${ }^{1,3}$ and Robyn O'Halloran ${ }^{1}$ \\ ${ }^{1}$ La Trobe University, Living with Disability Research Centre, Melbourne, Australia \\ ${ }^{2}$ Repatriation General Hospital, Speech Pathology Department, Adelaide, Australia \\ ${ }^{3}$ Summer Foundation, Box Hill, Victoria, Australia
}

$\boldsymbol{R}$ ackground and aims: Although changes within interpersonal relation$B$ ships have a substantial impact on the quality of life, a few authors have focused on how these changes are experienced by people with aphasia. The aim of this study was to systematically identify contemporary research focussing on the experience of people with aphasia in close personal relationships and to conduct a thematic analysis of the findings across these studies.

Method: Using scoping review methodology, four databases (CINAHL/EBSCO, MEDLINE/PubMed, Embase, PsycINFO) were systematically searched yielding 472 articles. Following title, abstract and full text 
review 21 studies remained. Data derived from these studies were charted, collated and thematically analysed for emerging themes.

Results: Most studies utilised interview methods and cross-sectional designs. Only seven directly examined the relationship experiences of people with aphasia. The remaining studies indirectly derived relationship focused responses through exploration of related constructs (living successfully, social participation, social communication, quality of life). Thematic analysis revealed four principal themes across the published findings. The first, relationships are important, captured the high value people with aphasia placed on relationships. Relationship input factors and output factors were reflected in the second and third themes respectively and the final theme, living with change, covered ongoing relationship challenges.

Conclusions: From the perspective of people with aphasia, relationships are not only central to positive aspects of everyday living but also challenging to maintain. Further research is needed to understand how relationships change over time and how to best support clients with aphasia to develop and maintain satisfying relationships.

Correspondence: Amy Ford; 3533872@students.latrobe.edu.au

\title{
Use of the Lubben Social Network Scale-18 to Measure Social Networks and Isolation Following Traumatic Brain Injury
}

\author{
Sophie Moore, ${ }^{1}$ Christine Migliorini ${ }^{1}$ and Libby Callaway ${ }^{1,2}$ \\ ${ }^{1}$ Department of Occupational Therapy, School of Primary and Allied Health Care, Monash University, \\ Frankston, Australia \\ ${ }^{2}$ Neuroskills Pty Ltd, Sandringham, Australia
}

\begin{abstract}
$B$ ackground and aims: Current literature describes long-term community integration, social engagement and social support following traumatic brain injury (TBI). However, few studies use an instrument that concurrently evaluates the quality of social ties, social engagement and relative levels of social isolation. The aim of this study was to trial use of a psychometrically sound, linguistically valid and easy to complete instrument designed to describe the social networks of adults with TBI, the Lubben Social Network Scale-18 (LSNS-18).

Methods: This cross-sectional study included 62 adult participants with TBI and high support needs (Care and Needs Scale (CANS) level $\geq 5$ ) residing in supported accommodation or their own homes with others. Two measures were used, the LSNS-18 to examine social networks and the CANS to measure level of support need. Social Isolation was calculated using a published algorithm. Results: Sixty per cent of study participants were deemed socially isolated. Type of residence and characteristics of co-residents was found to impact both size and quality of social networks. People with TBI living with other residents who also experienced disability were $\sim 87$ times more likely to be socially isolated than those living with people who did not experience disability.

Conclusions: This study demonstrated that living with TBI and high support needs is a significant liability to the size and quality of a person's social ties. The type of residence and whether the person with TBI lives with others with disability may also influence social networks. This initial study provides directions for future research and disability housing policy.
\end{abstract}

Correspondence: Sophie Moore; sophie.moore@monash.edu 


\section{Using Technology in Rehabilitation after TBI for Cognitive-Communication Skills}

Melissa Brunner, ${ }^{1}$ Bronwyn Hemsley, ${ }^{1}$ Stuart Palmer ${ }^{2}$ and Leanne Togher $^{3}$

${ }^{1}$ Speech Pathology, Faculty of Education and Arts, University of Newcastle, Newcastle, Australia

${ }^{2}$ Faculty of Science, Engineering \& Built Environment, Deakin University, Geelong, Australia

${ }^{3}$ Speech Pathology, Faculty of Health Sciences, University of Sydney, Sydney, Australia

$\boldsymbol{R}$ ackground and aims: For people with cognitive-communication disabilB ities after a traumatic brain injury (TBI), little is known about how they experience communication in digital or online environments. Social media interactions offer another way for people in society to connect with family, friends, and the community, which creates potential for people with TBI to increase their social participation. In considering the use of social media in cognitive-communicative rehabilitation, it is important to consider all types of technology used with people after TBI.

Method: A systematic, integrative literature review of communication technology in TBI rehabilitation and cognitive-communication was conducted.

Results: The 95 studies included in the review investigated three major types of communication technologies: (a) Assistive Technologies (AT) $(n=6)$, including Augmentative and Alternative Communication (AAC) $(n=27)$; and (b) Information and Communications Technologies (ICT) $(n=69)$. Multiple factors relating to use of technology were identified which informed the development of an evidence-based framework for incorporating technology into speech pathology clinical practice and research. The proposed model and its application to clinical practice and future research will be further explored in the presentation.

Conclusions: The proposed model provides an evidence-based framework for researchers and clinicians to consider when incorporating technology into cognitive-communicative rehabilitation for people after TBI. Using the model, researchers and clinicians might be able to better address the needs of people following a TBI, enabling them to use technology and online communities to increase their communication, social participation, and social support.

Correspondence: Melissa Brunner; melissa.brunner@uon.edu.au

\section{\#Hashtag TBI: Communication and Networking in Twitter by Traumatic Brain Injury communities}

Melissa Brunner, ${ }^{1}$ Bronwyn Hemsley, ${ }^{1}$ Leanne Togher, ${ }^{2}$ Stephen Dann ${ }^{3}$ and Stuart Palmer $^{4}$

${ }^{1}$ Speech Pathology, Faculty of Education and Arts, University of Newcastle, Newcastle, Australia

${ }^{2}$ Speech Pathology, Faculty of Health Sciences, University of Sydney, Sydney, Australia

${ }^{3}$ College of Business and Economics, Australian National University, Canberra, Australia

${ }^{4}$ Faculty of Science, Engineering \& Built Environment, Deakin University, Geelong, Australia

$\boldsymbol{R}$ ackground and aims: Twitter is used by millions of people worldwide $\mathcal{B}$ as a microblogging social networking site. Currently, no studies report on Twitter data, Twitter networks, or tweet content of people with Traumatic Brain Injury (TBI) or cognitive-communication impairment. Therefore, the aim was to gather and analyse TBI-related tweets to provide an understanding of how Twitter is being used to share information about TBI.

Method: Tweets tagged with TBI-related terms (\#hashtags) were captured in Twitter daily, for 1 month. Tweets were analysed using multiple methods: (a) A content classification framework; (b) Inductive qualitative coding of tweet content; and (c) Computational analysis of Twitter networks and tweet text content. 
Results: From 1st to 31st March 2016, 52581 tweets were captured. With 23382 tweets excluded, 29199 tweets sent by 893 users remained in the dataset for analysis. Preliminary content analysis revealed that TBI-related tweets were used to discuss and share information around: (i) health issues; (ii) awareness; (iii) life experiences after TBI; (iv) recovery and rehabilitation; (v) popular issues surrounding sport and TBI; and (vi) inspiration.

Conclusions: Analysis revealed that Twitter is being used for a variety of purposes when communicating about TBI by a large number of Twitter users, including several disclosing having a TBI. Results suggest that Twitter might be an important social media to consider in TBI rehabilitation, given the inclusion of people with TBI in the Twitter communities located in the data and the TBI-related information exchanged in Twitter networks. Implications for TBI cognitive-communication rehabilitation will be discussed.

Correspondence: Melissa Brunner; melissa.brunner@uon.edu.au

\section{Nutrition Management of Acquired Brain Injury Rehabilitation: A Cross-Sectional Survey}

Jane Stewart, Emily Butler and Georgina Konidaris

The Acquired Brain Injury Rehabilitation Centre, Alfred Health, Melbourne, Australia

$\boldsymbol{R}$ ackground and objectives: Adequate nutrition is integral in the rehabilitation of patients with an Acquired Brain Injury (ABI) (Welch-West et al., 2013). However, there is a lack of nutrition practice guidelines for ABI rehabilitation. This survey aims to document current practice in the nutritional management of patients at Specialist ABI Rehabilitation Centres in Australia, New Zealand and selected international reference centres.

Method: A purposive sample of 23 dietitians working in ABI Rehabilitation was surveyed using an online questionnaire that examined current nutritional management practices and dietetic staffing levels.

Results: No service met the dietetic staffing level recommended by the Australasian Faculty of Rehabilitation Medicine (AFRM). In the inpatient setting, screening for malnutrition and unintentional weight gain were routinely performed, however there was no consistency in the screening tools used. Oral nutrition support and enteral nutrition via gastrostomy were common. Variation in nutrition prescription was noted and most dieticians deemed further research into this area is required. Weight and body mass index were the predominant outcome measures used to evaluate efficacy of nutrition intervention. Nutrition services in community rehabilitation and Transitional Living Service were limited. No service screened for malnutrition and only one third screened for unintentional weight gain.

Conclusions: Nutritional management following ABI varies between services. Dietetic staffing levels fall below AFRM recommendations. These findings indicate a need for more research; and the development of evidence-based guidelines which are prescriptive and clearly define the role of the dietician in the management of patients undergoing ABI rehabilitation.

Correspondence: Jane Stewart; jane.stewart@alfred.org.au 
Concurrent Session 11 - How to Session

\title{
How to Develop and Implement an Interdisciplinary Approach to Behaviour Management in Inpatient ABI Rehabilitation - The Caulfield Experience
}

\author{
Luke Delaney, ${ }^{1}$ David Barton, ${ }^{1}$ Irish Garcia, ${ }^{1}$ Jacqui Morarty, ${ }^{1}$ Mithu Palit, ${ }^{1}$ Anna \\ Uliando ${ }^{1}$ and Joanne Wrench ${ }^{2,3}$ \\ ${ }^{1}$ Caulfield ABI Rehabilitation Service, Alfred Health, Melbourne, Australia \\ ${ }^{2}$ Royal Talbot Rehabilitation Centre, Austin Health, Melbourne, Australia \\ ${ }^{3}$ Melbourne School of Psychological Science, University of Melbourne, Parkville, Australia
}

\begin{abstract}
C ynopsis of Session: A specialist statewide ABI Rehabilitation Service was $\mathcal{N}$ opened at Caulfield Hospital in 2014. A key imperative when constructing the service was to develop strategies to manage Behaviours Of Concern (BOC) - most especially on the inpatient ward. The ABI team is interdisciplinary and works to the standard that "All Care is Everyone's Responsibility". This includes management of BOC. This session will describe the approach taken to integrate management of $\mathrm{BOC}$ into the core business of the rehabilitation service.
\end{abstract}

\section{How to Provide Community-Based Behaviour Management Support to People with Acquired Brain Injury}

Jan Loewy, Jenny Todd, Samantha Burns, Kathryn Hoskin, Genevieve McMahon, Adrian Kamer and Harriet Downing

Diverge Consulting, Melbourne, Australia

ynopsis of session: This session will discuss models of community-based $\mathcal{N}$ behaviour management, the differences between community-based and hospital-based settings, and use case studies (one child and one adult) to explore effective approaches and techniques. Practical resources for behaviour intervention will also be provided.

\section{Concurrent Session 12}

\section{Recall of Autobiographical Memories Following Severe Paediatric Traumatic Brain Injury: Evidence of a Selective Deficit}

Suncica Lah, ${ }^{1,2}$ Louise Parry, ${ }^{3}$ Chloe Gott, ${ }^{1}$ Carly Black, ${ }^{1}$ Adrienne Epps ${ }^{3}$ and Michael Gascoigne ${ }^{1,2}$

${ }^{1}$ School of Psychology, The University of Sydney, Sydney, NSW, Australia

${ }^{2}$ ARC Centre of Excellence in Cognition and its Disorders, Macquarie University, Sydney, NSW, Australia

${ }^{3}$ Brain Injury Rehabilitation Program, Rehab2Kids, Sydney Children's Hospital, Randwick, NSW, Australia

$\boldsymbol{R}$ ackground and aims: Autobiographical memory (AM), which involves recall of events that contain semantic and episodic details, develops through childhood and adolescence. Traumatic brain injury disrupts the very cognitive skills and brain networks that underpin AM. The aim of this study is to examine the impact of paediatric traumatic brain injury (TBI) on AM, which is currently unknown. 
Method: This cross-sectional study included children with severe TBI ( $n=$ $14)$ and healthy control participants $(n=20)$ comparable in age and sex. All participants completed the Child Autobiographical Interview and a battery of neuropsychological tests.

Results: Autobiographical events provided by children with TBI contained significantly fewer episodic (but not semantic) details compared to healthy control children $(U(59)=209.50, p=.04, d=0.56)$. These deficits in recall of episodic details emerged as the age at assessment increased (rho $=0.68, p$ $<.001)$. Moreover, recall of fewer event details was related to poorer verbal memory for stories ( $r h o=.82, p<.001$; but not for word pairs) and lower FSIQ $(r h o=.60, p=.02)$ in the TBI group. The deficits in recall of episodic details, however, were eliminated when specific prompts were provided.

Conclusions: Our study reveals that children who have sustained severe TBI are at risk of selective deficits in recall of AM, with deficits emerging at an older age. This is important, as AM is purported to guide our behaviour, play a significant role in social interaction and provide a sense of self-continuity. Implications of these deficits for adaptive living and approaches to remediation will be discussed.

Correspondence: Suncica Lah; suncica.lah@sydney.edu.au

\title{
Guidelines for Speech, Language and Swallowing Management Following Paediatric TBI
}

Cristina Mei, ${ }^{1}$ Vicki Anderson, ${ }^{1-3}$ Mary-Clare Waugh, ${ }^{4}$ Louise Cahill ${ }^{5}$ and Angela Morgan $^{1,2}$ on behalf of the TBI guideline development group ${ }^{\mathrm{a}}$

\author{
${ }^{7}$ Murdoch Childrens Research Institute, Melbourne, Australia \\ ${ }^{2}$ University of Melbourne, Melbourne, Australia \\ ${ }^{3}$ Royal Children's Hospital, Melbourne, Australia \\ ${ }^{4}$ Children's Hospital at Westmead, Sydney, Australia \\ ${ }^{5}$ Lady Cilento Children's Hospital, Brisbane, Australia
}

$\boldsymbol{B}$ ackground and aims: Evidence-based guidelines aim to enhance patient outcomes and consistency of care. Yet they are rarely used for the management of communication and swallowing disorders following paediatric TBI. We aimed to improve clinical care of this population by developing evidenceand consensus-based recommendations for the management of speech, language and swallowing disorders during the first year of rehabilitation.

Method: A multidisciplinary committee consisting of expert health professionals and consumers was convened to develop recommendations. Systematic review evidence was appraised for the formulation of evidence-based recommendations, created using the National Health and Medical Research Council statement form. Consensus-based recommendations were devised using a three-round Delphi survey completed by the committee. The criterion for agreement was $80 \%$.

Results: Consensus-based and, where sufficient research was available, evidence-based recommendations were formed to guide management of speech, language and swallowing disorders, including: prediction of these disorders, health professional team required, optimal timing of assessment, assessment tools, intervention strategies, commencement of treatment, and key information to support parents.

Conclusions: Key recommendations include screening of children with moderate and severe TBI for these disorders acutely using appropriate informal and formal measures to guide intervention. In particular, patients with severe TBI and prolonged ventilation are an at risk group, and should be considered 
for early referral to speech pathology to support timely diagnosis and management. The developed recommendations provide a basis for the systematic management of communication and swallowing disorders that can be refined as new high-quality evidence emerges.

Correspondence: Angela Morgan; angela.morgan@mcri.edu.au

a Jeanette Baker, Consumer, Australia; Katie Banerjee, Children's Hospital at Westmead, New South Wales (NSW); Mandy Beatson, Auckland District Health Board, New Zealand; Candice Brady, Children's Hospital at Westmead, NSW; Kate Brommeyer, Royal Children's Hospital (RCH), Victoria; Petrea Cahir, RCH, Victoria; Cathy Catroppa, Murdoch Childrens Research Institute, Victoria; Cynthia Christianto, Sydney Children's Hospital, NSW; Suzi Drevensek, Children's Hospital at Westmead, NSW; Donna Fallon, Townsville Hospital, Queensland; Jane Fong, Women's and Children's Hospital, South Australia; Rob Forsyth, Newcastle University, England; Matthew Frith, Hunter New England Health, NSW; Patricia Grillinzoni, Consumer, Australia; Flora Haritou, RCH, Victoria; Sophie Huntley, RCH, Victoria; Tamara Kelly, Novita Children's Services, South Australia; Kate Osland, Children's Hospital at Westmead, NSW; Jessica Palmer, Townsville Hospital, Queensland; Claire Radford, Lady Cilento Children's Hospital, Queensland; Damien Roberts, $\mathrm{RCH}$, Victoria; Adam Scheinberg, Victorian Paediatric Rehabilitation Service, Victoria; Jillian Steadall, RCH, Victoria.

\title{
'Is this, is this the right path?'- Exploring Factors that Contribute to Students' Educational Choices Following Brain Injury.
}

\author{
Margaret Mealings, ${ }^{1,2}$ Jacinta Douglas ${ }^{1,3}$ and John Olver ${ }^{2,4}$ \\ ${ }^{7}$ School of Allied Health, La Trobe University, Bundoora, Australia \\ ${ }^{2}$ Epworth Rehabilitation, Richmond, Australia \\ ${ }^{3}$ Summer Foundation, Box Hill, Australia \\ ${ }^{4}$ Epworth Monash Rehabilitation Medicine Unit, Richmond, Australia
}

$\boldsymbol{R}$ ackground and objectives: Participating in education is a valued life goal for young people. Following TBI, many students experience significant challenges in achieving this goal. Whilst clinicians and educators have worked to develop processes for helping students transition from rehabilitation to education; difficulties, variability and inconsistency still occur. Although expert opinion is well represented in the literature, relatively little attention has been paid to the lived experience of students themselves. This project is part of a larger study designed to gain understanding of how students with TBI perceive their educational experiences over time, during secondary or tertiary schooling. In this paper we aim to explore students' perspectives about factors that influence their educational choices over time: returning to, during, and leaving their course.

Method: The data for this project are drawn from a longitudinal, qualitative investigation in which 12 students (aged 17-32 years) completed up to three indepth interviews over a period of 4-15 months during their course participation. A total of 30 interviews were analysed using grounded theory methods.

Results: Four themes of 'self', 'changes', 'study actions and processes', and 'supports' emerged as key components in understanding students' experiences in education. Students' educational choices were influenced by a combination of factors within each of these areas. Factors changed over time, reflecting a dynamic and interactive process for individual students.

Conclusions: By understanding factors that influence students' choices during their educational experience, rehabilitation clinicians may be better able to 
identify appropriate strategies to guide students in making their educational decisions.

Correspondence: Margaret Mealings; margaret.mealings@epworth.org.au

\section{Promoting Self-Management of Health}

Amy O'Brien

Brightwater Care Group, Perth, WA, Australia

$B$ ackground and aims: In Australia evidence suggests people with a disability have relatively poor access to health promotion programs (AIHW, 2010). People with acquired brain injury (ABI) in particular report an average of 4.1 chronic conditions in addition to their primary disability, compared to an average 2.6 for other disability groups and 1.1 for the general population (O'Rance, 2007). The introduction of the National Disability Insurance Scheme (NDIS) has created an emphasis on planning and self-management, heightening the importance of providing people with a disability accessible tools for managing their short and long term health and wellbeing. The HealthyMe Toolkit is a health passport-style document, constructed with the aim of supporting self-management of health for people with ABI.

Method: An environmental scan and a review of similar health passportstyle documents used internationally guided toolkit development. Twenty-two clients trialled the toolkit after several introductory sessions and were followed up at six and 12 week intervals. The Patient Activation Measure (PAM) was used to measure client confidence.

Results: Residents who trialled the HealthyMe Toolkit (and at least one other health promotion intervention) reported increased confidence in leading a healthy life and managing health conditions at the conclusion of the intervention period. Participants who had the least confidence in managing their health conditions initially, showed the greatest increase in confidence.

Conclusions: People with ABI who have low confidence in their ability to manage their health may benefit from using a health passport-style document as a means of tracking and planning health and wellbeing.

Correspondence: Amy O’Brien; amy.obrien@brightwatergroup.com

\section{Gimme a Break}

Sarah J. Lillas, E. Jean C. Hay-Smith and William Levack

Rehabilitation Teaching \& Research Unit, University of Otago, Wellington, New Zealand

$\boldsymbol{R}$ ackground: Traumatic Brain Injury (TBI) is common. It temporarily or permanently limits a person's ability to return to pre-injury activities, including work. The most prevalent TBI symptoms impacting a successful return to work is fatigue. To address an apparent research gap, regarding successful strategies for overcoming fatigue to allow a return to work, this study asked 'How do people manage fatigue, that they attribute to TBI, to enable them to conduct paid work'.

Method: This qualitative descriptive study recruited and interviewed seven participants (aged 24-66 years), about their experiences of returning to work with fatigue. All participants had returned to their pre-injury employment, with time since injury ranging from 6 to 22 months. The semi-structured interview schedule included questions on difficulties experienced at work due to fatigue, and adjustments made to manage fatigue at work. Transcribed interviews were thematically analysed. 
Result: All participants used breaks at work to manage their fatigue. These ranged from 'hidden breaks' where activity was minimally disrupted, to 'mini breaks' consisting of a short removal from activity to 'long breaks' that impacted on the activity. Participants described a process of learning about their 'stop' point and what type of break was needed when. This choice depended on their fatigue level, perceptions of how others viewed them and perceived work expectations.

Conclusions: Breaks were essential for success in returning to work after a TBI. People need support to identify their stop point, work through types of breaks available to them and problem solve barriers to taking breaks.

Correspondence: Sarah J. Lillas; sarah.lillas@xtra.co.nz

\title{
Sensorimotor and Physiological Indicators of Recovery in Mild Traumatic Brain Injury: A Systematic Review
}

\author{
Olivia A. Galea, ${ }^{1}$ Michelle A. Cottrell, ${ }^{1}$ Julia M. Treleaven ${ }^{1}$ and Shaun P. O'Leary ${ }^{1,2}$ \\ ${ }^{1}$ Division of Physiotherapy, School of Health and Rehabilitation Sciences, The University of Queens- \\ land, Brisbane, Australia \\ ${ }^{2}$ Department of Physiotherapy, The Royal Women and Brisbane Hospital, Brisbane, Australia
}

$\boldsymbol{R}$ ackground and aims: Resolution of neurocognitive deficits and symptom reproduction following Mild Traumatic Brain Injury (MTBI), otherwise known as Concussion, has been reported to occur within the first 7-10 days post injury in an adult population. However, there is some evidence to suggest that sensorimotor and physiological changes may persist even in the subacute period ( 28 days to 6 months). The aim of this study was to provide a synthesis of the best available evidence examining the persistence of sensorimotor and physiological changes in MTBI.

Method: Databases; PubMed, PscycINFO, SPORTDiscus, MEDLINE, CINAHL and Embase were searched between March and November 2016. Inclusion criteria established a priori was used to identify eligible cohort and case control studies. Reference lists of eligible studies were also searched. Critical appraisal utilised a modified version of the Downs and Black quality assessment tool. The American Association of Neurology classification of evidence matrix was used to establish level of evidence.

Results: Nineteen articles eligible for inclusion achieved a mean preliminary quality assessment score of 14.37 out of a possible 22 points $(S D=1.86)$. Thirteen studies demonstrated level one to four evidence for the persistence of sensorimotor and physiological deficits, and six for the absence of sensorimotor dysfunction in the subacute to chronic time period.

Conclusion: Moderate to strong level of evidence exists to support the persistence of sensorimotor and physiological, but not vestibular, changes in the subacute period following MTBI. The identification of these persisting changes has ramifications for the long-term management planning of some individuals following MTBI.

Correspondence: Olivia A. Galea; o.galea@uq.edu.au, olivia.galea@ uqconnect.edu.au 


\title{
Decompressive Craniectomy for Severe Traumatic Brain Injury: Is Life Worth Living?
}

Stephen Honeybul, ${ }^{1}$ Courtney Janzen, ${ }^{2}$ Kate Kruger $^{2}$ and Kwok M. $\mathrm{Ho}^{3}$

${ }^{1}$ Department of Neurosurgery, Sir Charles Gairdner Hospital and Royal Perth Hospital

${ }^{2}$ Department of Occupational Therapy, Sir Charles Gairdner Hospital

${ }^{3}$ Department of Intensive Care Medicine, Royal Perth Hospital and School of Population Health, University of Western Australia, Perth, Western Australia, Australia

\begin{abstract}
$\boldsymbol{B}$ ackground: This provocative study completed in partnership with Neurosurgeons and Occupational Therapists questions the benefit of decompressive craniectomy with severe traumatic brain injury (TBI) given the considerable impact on activity and participation with limited research into how acceptable this is.

Objectives: This study assessed the long-term functional outcome, quality of life and retrospective consent of patients who had survived following decompressive craniectomy for severe TBI.

Method: Thirty nine patients were assessed beyond 3 years, who were adjudged either severely disabled or in a vegetative state at 18 months after decompressive craniectomy. Outcome measures utilised were the Extended Glasgow Outcome Scale, modified Barthel Index (mBI), Zarit Burden Interview, 36-Item Short-Form Health Survey (SF-36) and a questionnaire regarding retrospective consent.

Results: Of the 39 patients, 20 were eligible. Five patients remained in a vegetative state from 18 months to 3 years. Fifteen patients remained severely disabled after 3 years. The patients' average daily activity per the $\mathrm{mBI}$ (Pearson correlation coefficient $[r]=-0.661, p=.01)$ and SF-36 physical score $(r=$ $-0.543, p=.037$ ) were inversely correlated with the severity of TBI. However, the SF-36 mental scores of the patients were reasonably high (median 46, interquartile range 37-52). The majority of patients and their next of kin would have provided consent for surgical decompression knowing the eventual outcome.

Conclusion: Decompressive craniectomies did not improve physical recovery beyond 18 months; however, many patients appeared to have recalibrated their expectations regarding what they believed to be an acceptable quality of life.

Correspondence: Courtney Colliss; courtney.colliss@health.gov.wa.au
\end{abstract}

\section{Key Considerations for Health Professionals Supporting People Experiencing Persistent Postural-Perceptual Dizziness (PPPD)}

\author{
Ann Sezier, Denise Taylor, Nicola Saywell, Nicola Kayes and Gareth Terry \\ School of Clinical Sciences, Auckland University of Technology, Auckland, New Zealand
}

\begin{abstract}
$B$ ackground: Persistent Postural-Perceptual Dizziness (PPPD) is a hard to diagnose disorder triggered by events affecting the vestibular system, such as brain injury. Despite a growing interest, knowledge about PPPD remains limited. In practice this often translates to patients feeling unsupported. Although the focus of this study was not specifically on brain injury, findings may be relevant to Health Professionals (HPs) working with this population.

Objectives: To explore the working-age adult's subjective experience of PPPD and its impact on social, personal and work life, for the purpose of generating points for consideration immediately applicable to practice.

Method: This qualitative exploratory study followed an Interpretive Descriptive approach. Inductive Thematic Analysis was used to analyse data from single, semi-structured, individual interviews with working-age adults $(n=8)$
\end{abstract}


affected by PPPD, purposively sampled from a community based specialist dizziness clinic in New Zealand.

Results: Three interrelated themes were constructed. 'It sounds like I am crazy', referred to invisibility of the condition and lack of understanding being detrimental to legitimacy and validation. 'I'm a shadow of my former self' referred to biographical disruption and altered self-identity, and 'How will I survive' referred to fluctuating coping processes.

Conclusions: Although diagnosis and treatment were highly important for legitimisation, care was only perceived as satisfactory if HPs showed interest in the individual's experiences and its impact on their life. This could be achieved by HPs Individualising support and paying attention to the person's context and priorities, exchanging knowledge, responding to fluctuating stages of coping, and considering co-existing conditions.

Correspondence: Ann Sezier, asezier@aut.ac.nz

\title{
HIV-Associated Neurocognitive Disorder: Establishing a Screening and Diagnostic Service for Hand in Tasmania
}

\author{
Clare Ramsden, Sigrid Denehey, Madeline Rowell and David Tuck \\ Tasmanian Health Service, Tasmania, Australia
}

\begin{abstract}
$B$ ackground and objectives: HIV-associated neurocognitive disorders (HAND) occur in approximately 50-60\% of people diagnosed with HIV (Zipursky et al., 2013). A clinical assessment pathway was developed to provide access to appropriate cognitive assessment for this cohort, based on Woods et al.'s (2009) clinical diagnostic decision tree, including assessment of functional impact and cognitive impairment.

Method: A cognitive assessment pathway for HAND was developed, including multidisciplinary assessment of activities of daily living and the International HIV Dementia Scale, a standardised cognitive assessment investigating cognitive domains of speed of information processing, working memory, executive function, verbal memory, naming, and visuoconstructional skills, and estimated premorbid intellectual function.

Results: In the pilot phase, 16 patients completed the standardised brief cognitive assessment. Average premorbid intellectual function was $101.6(n=$ 14, range 84-116), and six patients met criteria of at least two performances below expectation; these subtests included measures of speed of information processing, working memory or executive dysfunction.

Conclusions: Following a structured cognitive assessment pathway, 6 of a total 16 patients were identified as meeting criteria for further cognitive assessment. Executive function was impaired in this subgroup, but there was no evidence of impaired learning which has been implicated in the literature. This difference may be related to the time of diagnosis and progression of neurocognitive impairments in this small sample.
\end{abstract}

Correspondence: Clare Ramsden; clare.ramsden@ths.tas.gov.au 


\title{
Risk Factors for Cognitive Impairment in Substance Users Attending Treatment
}

Nicole Ridley, ${ }^{1}$ Lauren Monds, ${ }^{1,2}$ Annie Malcolm, ${ }^{1}$ Kate Finsterer, ${ }^{1}$ Withall Adrienne, ${ }^{3}$ Brian Draper $^{4,5}$ and Nicholas Lintzeris ${ }^{1,2}$

${ }^{1}$ Drug and Alcohol Services, South Eastern Sydney Local Health District, NSW Health, Australia

${ }^{2}$ Discipline of Addiction Medicine, Central Clinical School, Sydney Medical School, University of Sydney, Sydney, Australia

${ }^{3}$ School of Public Health \& Community Medicine, University of NSW, Sydney, Australia

${ }^{4}$ School of Psychiatry, University of NSW, Sydney, Australia

${ }^{5}$ Academic Department of Old Age Psychiatry, Prince of Wales Hospital Randwick, Sydney, Australia

$\boldsymbol{R}$ ackground and objectives: Cognitive impairment in individuals with substance-use disorders has a recognised impact on treatment outcomes. However, the extent of cognitive impairment, and the risk factors that predict cognitive outcome in this group, is not clear. The aim of this project was to establish the level of cognition and identify risk factors for cognitive impairment in a convenience sample of 120 clients attending South-Eastern-Sydney Drug and Alcohol services for opioid substitution treatment (OST).

Method: Clients were assessed using a validated cognitive screening instrument (the Montreal Cognitive Assessment; MoCA). In addition a new risk factor assessment questionnaire was developed by researchers and clinicians, and administered to clients. This gathered information relevant to drug and alcohol clients (including history of substance use, concussions, neurological events, loss of consciousness, physical and mental health, and client self-report of cognition and functional skills).

Results: Participants had on average 9.6 years of education; mean age was 44.5 years. The average MoCA score was 24.6 , and $53 \%$ of the sample scored below the validated cut-score $(<26)$ for suspected cognitive impairment. $52 \%$ had experienced loss of consciousness for $>30$ minutes from various aetiologies. The specific risk factors best predictive of cognitive deficits in this group are discussed.

Conclusions: The results demonstrate the high prevalence of cognitive deficits in OST clients. These clients have multiple risk factors for cognitive impairment. Cognitive screening should form part of routine clinical treatment, and specific risk factors related to cognitive outcome can be used to flag clients vulnerable for cognitive deficits.

Correspondence: Nicole Ridley; Nicole.ridley@ health.nsw.gov.au

\section{Concurrent Session 13}

\section{TASIT-S: An Abbreviated Version of the Awareness of Social Inference Test. New Normative Data for Australian and US Speakers}

\author{
Skye McDonald, ${ }^{1}$ Samantha K. Allen, ${ }^{1}$ Cynthia Honan, ${ }^{2}$ Christine Padgett, ${ }^{2}$ Fiona \\ Kumfor, ${ }^{3}$ Olivier Piguet ${ }^{3}$ and Jessica Hazelton ${ }^{3}$ \\ ${ }^{1}$ School of Psychology, UNSW Australia \\ ${ }^{2}$ Psychology, Department of Medicine, University of Tasmania \\ ${ }^{3}$ Brain and Mind Centre, The University of Sydney
}

\footnotetext{
$\boldsymbol{B}$ ackground: It is increasingly recognised that social cognition should form part of our cognitive and communication assessments of people with brain disorders. The Awareness of Social Inference Test (TASIT) uses naturalistic videos to assess emotion perception, theory of mind and understanding of pragmatic inference (like sarcasm). While widely used, TASIT is
} 
lengthy to administer. We recently developed a short version based on Rasch analysis with 10, 9 and 9 items for Parts 1, 2 and 3 respectively. In this paper we report on normal adult performance on TASIT-S, comparing USA and Australian speakers.

Method: 181 USA speakers (mean age 35) completed TASIT-S online (via mTurk). Their performance was compared to 161 Australian speakers (mean age 38) who were administered TASIT-S face to face. An additional 65 Australian adults were included to examine the effects of age on performance.

Results: USA speakers performed slightly better on emotion recognition and judging sincere items than Australians. Australians were slightly better detecting lies but overall performance across cultures was comparable. Australians aged over 60 (mean age 68) were uniformly poorer than younger adults (mean age 36) on all parts of TASIT-S. There was no effect of gender. More educated adults performed better on TASIT-S part 2.

Conclusions: Despite having to understand actors with Australian accents, this study shows that USA speakers perform comparably to Australians. The main influence on TASIT-S performance was age and, to a less extent, education. These norms provide a useful framework for using TASIT-S in clinical settings.

Correspondence: Skye McDonald; s.mcdonald@unsw.edu.au

\title{
Theory of Mind in People with Cognitive-Communication Disorder after Right Hemisphere Stroke
}

\author{
Petrea Cornwell, ${ }^{1,2,3}$ Stephanie Vierboom ${ }^{2}$ and Ronelle Hewetson ${ }^{2,3}$ \\ ${ }^{1}$ School of Applied Psychology and Menzies Health Institute Queensland, Griffith University, Brisbane, \\ Australia \\ ${ }^{2}$ School of Allied Health Sciences, Griffith University, Gold Coast, Australia \\ ${ }^{3}$ The Prince Charles Hospital Metro North Hospital and Health Service, Brisbane, Australia
}

$\boldsymbol{R}$ ackground and aims: Daily communication relies on the ability to make B social inferences about speaker intention, attitude and meaning. Deficit in theory of mind (ToM) is common after right hemisphere stroke (RHS). This study aimed to (1) describe ToM in people with cognitive-communication disorder (CCD) post RHS including differential patterns of performance, and (2) compare ToM abilities to an age and gender matched control group.

Method: Twenty-four participants (12 RHS, 12 controls) that didn't differ significantly in age (range 52-84 years) or education $(\mathrm{p}>.05)$ completed the two social inference tasks of The Awareness of Social Inference Test (TASIT-R). A summary score combining results from two TASIT-R tasks was created for each participant. Descriptive statistics summarised participant performance on the TASIT-R for each social inference task and summary score. Comparison between the two groups were completed using the Mann-Whitney Test, significance level set at $\mathrm{p}<.05$.

Results: Using clinical cut-off scores participants' performance on the TASIT$\mathrm{R}$ revealed impairments in ToM in all participants with CCD across both of the social inference tasks. It was noted that $50 \%$ of the control group also fell below the clinical cut-off on both tasks. Statistical between-group comparisons revealed a significant between-group difference for the summary score $(\mathrm{p}=$ $.003)$, social inference minimal task $(\mathrm{p}=.037)$, and social inference enriched task $(\mathrm{p}=.003)$.

Conclusions: Deficits in ToM were evident in all participants with CCD. Further examination of older peoples' performance on the TASIT-R is indicated based on control group performance.

Correspondence: Petrea Cornwell; p.cornwell@ griffith.edu.au 


\title{
The Brief Assessment of Social Skills (BASS) in People with a Diagnosis of Dementia
}

Michelle Kelly ${ }^{1}$ and Skye McDonald ${ }^{2}$

${ }^{1}$ School of Psychology, University of Newcastle, Newcastle, Australia

${ }^{2}$ School of Psychology, University of New South Wales, Sydney, Australia

\begin{abstract}
$\boldsymbol{B}$ ackground and objectives: Changes in social functioning in people with dementia are common, particularly with the advancement of the disease process. Despite this, there are no well validated tests available for the assessment of social skills in this population. We have developed the Brief Assessment of Social Skills (BASS) for this purpose.

Method: The BASS was administered to 24 people with a diagnosis of dementia $(M$ age $=81.71: 16 \mathrm{~F}, 8 \mathrm{M})$ and 24 controls $(M$ age $=78.17: 13 \mathrm{~F}, 11 \mathrm{M})$. Participants completed several other assessments of general and social cognition to allow for examination of the validity and reliability of the BASS.

Results: Participants with a diagnosis of dementia performed significantly worse on the BASS than the Control group $(p<.001)$. Performance on the BASS was significantly related to age $(r=-.417, p=.003)$, age at retirement $(r=.337, p=.02)$, and performance on cognitive screening $(r=.812, p<.001)$, however was not affected by years of education $(p>.05)$, nor were there gender differences $(p>.05)$. Significant strong $(p ' s<.001)$ positive correlations were obtained between performance on the BASS and established tests of social cognition.

Conclusions: The emergence of social cognition impairments in people with dementia puts incredible strain on family and paid care providers. With further collection of normative data and evidence for the validity and reliability of the BASS, it is hoped that the BASS will be included in routine assessment. This will provide carers with information to better manage changes in social skills as they arise, and in turn, facilitate longevity of the caring relationship.
\end{abstract}

Correspondence: Michelle Kelly; Michelle.Kelly@ newcastle.edu.au

\section{Feasibility and Efficacy of Brief Computerized Training to Improve Emotion Recognition in Premanifest and Early-Symptomatic Huntington's Disease}

Clare Kempnich, Dana Wong, Nellie Georgiou-Karistianis and Julie Stout

Monash Institute of Cognitive and Clinical Neurosciences, Monash University Clayton Campus, Australia

$\boldsymbol{R}$ ackground and objectives: Emotion recognition is impaired before clin3 ical diagnosis of Huntington's disease (HD), and may impact patients' social interactions. Although emotion recognition deficits have been improved using computerized methods in other conditions such as schizophrenia, to date there have been no attempts to systematically improve emotion recognition performance in HD. We conducted a small study of the feasibility and efficacy of computerized training of emotion recognition in HD.

Method: 22 individuals with premanifest or early-symptomatic HD were randomly assigned to the training or control group. The training group used an online emotion recognition training program (MicroExpression Training Tool) twice weekly for 4 weeks, and completed training adherence measures. All participants completed measures of emotion recognition at baseline, and after training.

Results: Participants in the training group completed on average seven of the eight sessions. Results also showed a significant group by time interaction 
indicating that the training program was associated with improved accuracy in emotion recognition for the training group only.

Conclusions: Our small study demonstrates that emotion remediation using an online training program is feasible in terms of training adherence, and effective in improving emotion recognition accuracy which opens up a potential new avenue for social intervention in HD. Further study with a larger sample size is needed to replicate our findings, and to investigate the durability and generalisability of training outcomes and their potential impact on patients' social function.

Correspondence: Clare Kempnich; clare.kempnich@monash.edu.au

\section{Concurrent Session 14}

\section{Revisiting the Case for Single Case Research}

John E. Pierce, ${ }^{1,2}$ Robyn O'Halloran, ${ }^{1}$ Leanne Togher ${ }^{3}$ and Miranda Rose ${ }^{1}$

${ }^{1}$ School of Allied Health, La Trobe University, Melbourne, Australia

${ }^{2}$ Speech Pathology, Cabrini Health, Melbourne, Australia

${ }^{3}$ Faculty of Health Sciences, University of Sydney, Sydney, Australia

$\boldsymbol{B}$ ackground and objectives: In recent years, the supremacy of the RanB domised Controlled Trial (RCT) in health research has been increasingly questioned. Researchers have challenged the traditional evidence hierarchy where RCTs are placed above all other methodologies, at times without regard for rigour.

In parallel, the role of Single Case Experimental Designs (SCEDs) is being recognised by a broader field of researchers. For example, the Levels of Evidence table from the Oxford Centre for Evidence Based Medicine now classifies the medical $\mathrm{N}$-of-1 design (a rigorous single case design) at Level 1 alongside systematic reviews.

Method: SCEDs can be used to investigate effectiveness of interventions in specific subpopulations whereas results from group designs can be difficult to generalise. In addition, participants who meet all inclusion criteria for RCTs may not be representative of the clinical population which often involves multiple comorbidities. These issues are highly pertinent to research in many brain impairment conditions due to the heterogeneity of these populations.

Results: This presentation argues for the utility of high quality SCEDs and outlines recent advances in their design and analysis, including an effect size measure (Tau-U), a methodological rating scale (RoBiNT), and recently published reporting guidelines (SCRIBE).

Conclusions: Recent developments in analysis, design, and reporting of SCEDs enhance their potential to produce high quality, readily implemented evidence.

Correspondence: John E. Pierce; pierce.john.e@gmail.com

\section{How Can a Pākehā (Non-Indigenous) Researcher Engage Families in a Way that is Practical, Effective and Respectful to Māori?}

Elisa Lavelle Wijohn

School of Clinical Sciences, AUT University, Auckland, New Zealand

$\mathcal{R}$ ackground and aims: Māori have disproportionately high rates of brain

$B$ injury, yet service delivery tends to be built on Western and individualistic paradigms which run counter to Māori values. Community organisations and 
individuals often experience research to be extractive and burdensome. In order to work respectfully and effectively in conducting research in a low-income predominantly Māori and Pacific community it was important to develop an appropriate methodology and sound processes. This paper will outline the values and principles of Māori-centred, Community-Based Participatory Research (CBPR) and demonstrate its effectiveness within a $\mathrm{PhD}$ project.

Method: A Māori-centred ethical framework was used to engage in CBPR with family members in South Auckland. This process was evaluated using pen and paper feedback, discussion processes and researcher journaling.

Results: A total of 19 people participated, as co-researchers, in 25 meetings over a period of 6 months. The group's work produced a well-received culturally relevant action project, in the form of a wānanga, that benefited the lives of co-researchers, wānanga attendees, and the wider community of families living with serious adult brain injury. In line with the CBPR principle of sustainability, the group's work continues as the Brain Injury Whānau Action Project.

Conclusion: Māori-centred CBPR is a practical and effective methodology that is respectful of Māori and delivers both a contribution to knowledge and direct benefits to participants and their community.

Correspondence: Elisa Lavelle Wijohn; elisa.wijohn@gmail.com

\title{
An Audit and Feedback Program Embedded into an ABI Rehabilitation Service Increased Adherence to Clinical Practice Guidelines
}

\author{
Laura Jolliffe, ${ }^{1}$ Natasha Lannin, ${ }^{1,2}$ Tammy Hoffmann, ${ }^{3}$ Jacqui Morarty, ${ }^{2}$ Elizabeth \\ O'Shannessy, ${ }^{2}$ Peter Hunter, ${ }^{2}$ Andrew Perta, ${ }^{2}$ Maria Crotty ${ }^{4}$ and Ian D. Cameron ${ }^{5}$ \\ ${ }^{\prime}$ LaTrobe University, Melbourne, Victoria, Australia \\ ${ }^{2}$ Alfred Health, Melbourne, Victoria, Australia \\ ${ }^{3}$ Bond University, Gold Coast, Queensland, Australia \\ ${ }^{4}$ Flinders University, Adelaide, South Australia, Australia \\ ${ }^{5}$ University of Sydney, Sydney, New South Wales, Australia
}

$\boldsymbol{B}$ ackground and aims: Clinical practice guidelines (CPGs) summarise $B$ research evidence and provide guidance for clinicians in an effort to reduce clinical variation and improve quality of care. CPGs, however, have limited effect unless they are implemented. The aim of this study was to evaluate the effects of an audit and feedback knowledge translation intervention on clinician adherence to acquired brain injury (ABI) rehabilitation CPGs.

Method: Using a periodic service review method, inpatient rehabilitation care was audited fortnightly for 46 weeks ( $n=61$ cases) against ABI rehabilitation CPGs ( $n=137$ observable criteria). Audit data were collected using medical record review, observation, and staff, patient and family interviews. Feedback sessions were facilitated fortnightly during which a summary of observed adherence to CPGs was presented and the multidisciplinary team discussed performance and identified potential solutions to address performance gaps. At the 3-month follow-up, $n=20$ randomly selected cases were audited to understand if adherence is maintained after feedback ceases.

Results: There was a significant improvement in adherence to CPGs during the intervention period (35\% improvement, 95\% CI 25 to 46) $(p=.0001)$. Three months after the audit and feedback intervention was ceased, clinician adherence fell to $73 \%$ (9\% reduction, $95 \% \mathrm{CI}-19$ to 0.5$)(p=.002)$ and there were variations in adherence across the guideline areas.

Conclusions: Brain injury rehabilitation may benefit from embedded audit and feedback programs where clinicians lead change initiatives. Findings suggest 
that to achieve sustainable change, the audit and feedback program needs to be incorporated into usual rehabilitation rather than be a research initiative.

Correspondence: Laura Jolliffe; 1.jolliffe@latrobe.edu.au

\title{
Goal Setting in Brain Injury Rehabilitation: Exploring the Process in Practice
}

\author{
Sarah Prescott, ${ }^{1}$ Jennifer Fleming ${ }^{1,2}$ and Emmah Doig ${ }^{1}$ \\ ${ }^{1}$ School of Health and Rehabilitation Sciences, The University of Queensland, Brisbane, Australia \\ ${ }^{2}$ Occupational Therapy Department, Princess Alexandra Hospital and the Centre for Functioning and \\ Health Research, Metro South Health District, Queensland Health, Brisbane, Australia
}

$\boldsymbol{R}$ ackground and aims: Goal setting in clinical practice is a highly individualised process, but research examining practice is limited. To address this need, we interviewed 22 multi-disciplinary clinicians working in brain injury and developed a goal setting practice framework to explain how achievable client-centred goals are developed. However, this framework was based on interview data and may reflect clinician's theoretical knowledge rather than goal setting processes used in routine practice. Therefore this study aimed to identify the goal setting processes used by clinicians to engage clients in goal setting, by exploring the application of the goal setting practice framework in routine practice.

Method: A qualitative observational study of clinical practice was employed. Participants were community dwelling clients with ABI, drawn from a hospitalbased outpatient service and community-based private practices. The communication exchange between multi-disciplinary clinicians and clients during routine goal setting was audio-recorded, transcribed verbatim and analysed using Framework Analysis. Strategies to enhance rigour included independent coding and fortnightly consensus meetings.

Results: A total of 66 goal setting sessions with 37 clients and 18 clinicians were analysed. The goal setting practice framework explains processes used in clinical practice, however additional strategies to establish trust were noted including communication to encourage social connection. Furthermore, communication was structured by clinicians when clients were unable to respond to direct questions. This involved using verbal statements to promote client self-reflection and understanding during all phases of the goal setting process. Conclusions: The identified strategies may be used to enhance client engagement in goal setting.

Correspondence: Sarah Prescott; s.prescott@uq.edu.au

\section{Concurrent Session 15 - How to Session}

\section{Translating Innovations in Discourse Assessment: A Practical Guide for Assessment of Cognitive and Social Communication in Adults with Acquired Brain Injury}

Joanne Steel, ${ }^{1,3}$ Elise Elbourn ${ }^{2,3}$ and Leanne Togher ${ }^{2,3}$

${ }^{7}$ Department of Speech Pathology, The University of Newcastle, Newcastle, Australia

${ }^{2}$ Department of Speech Pathology, The University of Sydney, Sydney, Australia

${ }^{3}$ Centre of Research Excellence in Brain Recovery: Moving Ahead, Australia

ynopsis of session: Discourse-level communication is commonly affected $\mathcal{N}$ after acquired brain injury (ABI). Recommended assessment practice for clients with $\mathrm{ABI}$ includes use of standardised tests and discourse assessment. Clinically, speech pathologists commonly assess communication at 
impairment level and perceive discourse analysis as time consuming and/or complex. However, there are relatively simple ways of evaluating discourse to explore cognitive and social communication impairments across the continuum from acute to community settings. This workshop aims to translate research knowledge on discourse assessment into clinical practice. Using techniques based on the Talkbank protocols, the presenters will discuss ways of approaching discourse assessment and outline how to use these clinically to inform treatment directions. There will be opportunity for participants to practise discourse analyses using real case examples. At the completion of the workshop, participants will be able to identify which types of discourse analysis are suitable for different subgroups/profiles of impairment. The discourse assessment techniques in this workshop can be employed with adults with TBI, stroke, dementia, and neurodegenerative diseases.

\title{
Concurrent Session 16 - How to Session
}

\section{Identifying Depression and Anxiety in People with Non-Traumatic Brain Injury}

lan Kneebone ${ }^{1}$ and Brooke Ryan ${ }^{2}$

${ }^{1}$ University of Technology Sydney, Sydney, Australia

${ }^{2}$ University of Queensland, Queensland, Australia

C ynopsis of session: Identifying mood disorders in people with non$\mathcal{N}$ traumatic brain injury (i.e., stroke, aneurysm) can be difficult. This is particularly true for people who have either a cognitive and/or communication disability that affects their ability to self -report on emotional state. This "how to" will consider both qualitative and quantitative means of detecting depression and anxiety in this population.

\section{Concurrent Session 17}

\section{Self-Awareness, Emotional Distress and Psychosocial Functioning in the First 6 Months After Hospitalisation Due to Traumatic Brain Injury}

\author{
Jennifer Fleming, ${ }^{1,2}$ Megan Geytenbeek, ${ }^{1,2}$ Emmah Doig $^{1}$ and Tamara Ownsworth ${ }^{3}$ \\ ${ }^{1}$ School of Health and Rehabilitation Sciences, The University of Queensland, Brisbane, Australia \\ ${ }^{2}$ Occupational Therapy Department, Princess Alexandra Hospital, Brisbane, Australia \\ ${ }^{3}$ School of Applied Psychology, Menzies Health Institute Queensland, Griffith University, Brisbane, \\ Australia
}

R ackground and aims: People with traumatic brain injury (TBI) may exhibit impaired self-awareness (ISA) which can compromise psychosocial reintegration following discharge from hospital. This study aimed to describe the occurrence of ISA after TBI and determine its association with emotional distress and psychosocial functioning over the first 6 months of community living.

Method: A prospective cohort design was used with data collection at discharge and 1, 3 and 6 months follow-up. Participants were 81 adults with TBI and their significant others. Self-awareness was measured using a discrepancy score generated from the Mayo-Portland Adaptability Index (MPAI-4) Ability Scale, and significant other's ratings of Item 20 on the MPAI-4. Other 
measures were the Depression Anxiety Stress Scale-21 and Sydney Psychosocial and Reintegration Scale.

Results: The discrepancy score method identified more cases of ISA than the single item rating by significant others. Using discrepancy scores, the occurrence of ISA was $69.1 \%$ at discharge, which reduced to $54.3 \%$ at 6 months follow-up. Higher levels of self-awareness were associated with heightened symptoms of anxiety and stress at discharge and stress 3-6 months later, and better psychosocial functioning at all time points. Participants with ISA had significantly poorer independent living skills at 6 months after controlling for injury severity.

Conclusions: Although self-awareness can increase stress levels for patients with TBI during reintegration into community living, it is also associated with better outcomes, indicating the importance of targeting ISA in rehabilitation.

Correspondence: Jennifer Fleming; j.fleming@uq.edu.au

\title{
The Mediating Role of Resilience among Family Members Supporting Relatives with Traumatic Brain Injury (TBI): A Structural Equation Modelling Approach
}

\author{
Grahame Simpson,, ${ }^{1,2}$ Malcolm Anderson ${ }^{3}$ and Maysaa Daher ${ }^{1,4}$ \\ ${ }^{1}$ Brain Injury Rehabilitation Research Group, Ingham Institute for Applied Medical Research, Liver- \\ pool, Sydney, Australia \\ ${ }^{2}$ Liverpool Brain Injury Rehabilitation Unit, Liverpool Hospital, Sydney, Australia \\ ${ }^{3}$ Avondale College of Higher Education, Sydney, Australia \\ ${ }^{4}$ Brain Injury Rehabilitation Directorate, Agency for Clinical Innovation, NSW Health, Australia
}

bjectives: A number of elements have been identified as mediating psychological adjustment amongst family members supporting relatives with TBI, but have rarely been tested within a single model. The current study tested the predictive and mediating relationships among personality style and coping, as mediated by self-efficacy, resilience, hope and social support, with a range of psychological outcomes among family members supporting relatives with TBI.

Method: Family members $(n=131)$ of relatives with severe TBI (posttraumatic amnesia $>1$ day) were recruited from 6 specialist rehabilitation services in New South Wales and Queensland. The study battery comprised the Eysenck Personality Questionnaire and Ways of Coping Questionnaire; the Connor-Davidson Resilience Scale, General Self-Efficacy Scale, Herth Hope Scale and Medical Outcome Study Social Support Survey; and four outcome measures (Caregiver Burden Scale, Mental Health sub-Scale-SF36, General Health Questionnaire, Positive and Negative Affect Scale). Structural Equation Modelling was used to test the hypothesised model.

Main results: The model fitted the data very well (goodness-of-fit indices: $\chi^{2}=58.521 ; p=.166 ; \mathrm{NFI}=0.934, \mathrm{IFI}=0.989, \mathrm{CFI}=0.998$ and RMSEA $=0.39)$. A substantial amount of variance $(63 \%)$ in resilience was accounted for by the joint influence of self-efficacy, coping strategies (problemfocused coping) and personality traits (extraversion). In terms of family member outcomes, resilience had a direct effect on positive affect in caregivers. Resilience also acted as a protective factor in relation to caregiver burden and increasing positive mental health among family members.

Conclusions: The research identified resilience as a key factor in the psychological adjustment process for family members.

Correspondence: Grahame Simpson; grahame.simpson@sswahs.nsw.gov.au 


\title{
Staying Connected after Brain Tumour: Changes in Social Networks and Relationship to Wellbeing after Brain Tumour
}

\author{
Lee Cubis, ${ }^{1,2}$ Tamara Ownsworth, ${ }^{1,2}$ Mark Pinkham, ${ }^{3,4}$ Melissa Legg ${ }^{2}$ and Suzanne \\ Chambers ${ }^{1,2,5}$ \\ ${ }^{1}$ School of Applied Psychology, Griffith University, Mt Gravatt, Australia \\ ${ }^{2}$ Menzies Health Institute Queensland, Griffith University, Gold Coast, Australia \\ ${ }^{3}$ School of Medicine, University of Queensland, Brisbane, Australia \\ ${ }^{4}$ Department of Radiation Oncology, Princess Alexandra Hospital, Woolloongabba, Australia \\ ${ }^{5}$ Cancer Council Queensland, Fortitude Valley, Australia
}

\begin{abstract}
$B$ ackground and aims: Social networks facilitate positive wellbeing in chronic illness; yet, for people with brain tumour, cognitive or physical impairments can occur and impair social integration. This mixed methods study aimed to: 1) examine associations between perceived functioning, social integration and psychological wellbeing in adults with primary brain tumour; and 2) explore changes in social networks and identify barriers/facilitators to social integration.

Method: Thirty-nine people with primary brain tumour (38\% benign; $8 \%$ low grade; 54\% high grade) aged 28-75 years were recruited from hospital and community services in Queensland. Participants completed a cognitive screener and self-report measures of cognitive and physical functioning (Functional Assessment of Cancer Therapy), social integration (structured interview \& Exeter Identity Transition Scale), depression, anxiety and life satisfaction. A purposive sample $(n=10)$ completed in-depth semi-structured interviews focused on the social impact of brain tumour.

Results: Controlling for age, participants reporting greater cognitive and physical impairments had poorer maintenance of pre-existing social groups ( $r=$ $-.42, p=.005)$. Participants with poorer maintenance of pre-existing social groups reported greater symptoms of depression $(r=-.55)$ and anxiety $(r=$ $-.44)$ and lower life satisfaction $(r=.33, p<.05)$ irrespective of their tumour type and global cognitive status. The qualitative analysis highlighted the diverse ways in which participants cope with social losses and strategies for maintaining and expanding social connections.

Conclusions: Disease-related barriers to social integration may need to be addressed to optimise wellbeing after brain tumour.
\end{abstract}

Correspondence: Lee Cubis; lee.cubis@griffithuni.edu.au

\section{Cognitive Behaviour Therapy for Sleep Disturbance and Fatigue Following Acquired Brain Injury: Pilot Randomised Controlled Trial}

\author{
Sylvia Nguyen, ${ }^{1,2}$ Adam McKay, ${ }^{1,2}$ Dana Wong ${ }^{1,2}$ and Jennie Ponsford ${ }^{1,2}$ \\ ${ }^{1}$ Monash Institute of Cognitive \& Clinical Neurosciences and School of Psychological Sciences, \\ Monash University, VIC, Australia \\ ${ }^{2}$ Monash-Epworth Rehabilitation Research Centre, Melbourne, VIC, Australia
}

$\boldsymbol{R}$ ackground and aims: Sleep disturbance and fatigue are common phenomena following acquired brain injury (ABI). Management of these symptoms are important targets in rehabilitation yet there is limited empirical investigation into effective interventions. A randomised controlled trial (RCT) was conducted to evaluate whether cognitive behaviour therapy (CBT) can be successfully adapted to alleviate sleep disturbance and fatigue in an ABI cohort.

Method: Participants ( $n=34)$ with history of traumatic brain injury (TBI) or stroke were randomly allocated to intervention (CBT) or treatment as usual 
(TAU). The intervention consisted of eight weekly therapy sessions with a neuropsychologist. CBT was delivered according to a manualised protocol and adapted to accommodate for cognitive impairments. Participants were reassessed at 2 and 4 months from baseline on measures of sleep, fatigue and mood.

Results: Relative to TAU, participants in the CBT group reported significantly greater improvements in sleep on the Pittsburgh Sleep Quality Index $(p=.018)$ and Insomnia Severity Scale $(p=.016)$. Reductions on fatigue was noted on different measures for TBI and stroke participants, the former evidencing significant change on the Brief Fatigue Inventory $(p=.016)$ and the latter on the Fatigue Severity Scale $(p=.02)$. Secondary improvements were found in depression on the Hospital Anxiety and Depression Scale $(p=.000)$. Gains were maintained at follow-up with large treatment effects.

Conclusions: Adapted CBT was effective in remediating sleep disturbance, fatigue and depression over TAU, with lasting effects for 2 months after therapy cessation. From this pilot study, CBT represents a promising intervention for these symptoms in persons with ABI.

Correspondence: Sylvia Nguyen; sylvia.nguyen@monash.edu

\title{
Concurrent Session 18
}

\section{Olfactory Function in Paediatric Traumatic Brain Injury: Recovery Patterns and Functional Implications}

\author{
Kathleen Bakker, ${ }^{1,2,3}$ Cathy Catroppa ${ }^{2,3,4,5}$ and Vicki Anderson ${ }^{2,3,4,5}$ \\ ${ }^{7}$ Victorian Paediatric Rehabilitation Service, Royal Children's Hospital, Melbourne, Australia \\ ${ }^{2}$ Murdoch Childrens Research Institute, Melbourne, Australia \\ ${ }^{3}$ Department of Paediatrics, University of Melbourne, Melbourne, Australia \\ ${ }^{4}$ Psychology Department, Royal Children's Hospital, Melbourne, Australia \\ ${ }^{5}$ School of Psychological Sciences, University of Melbourne, Melbourne, Australia
}

$\boldsymbol{R}$ ackground and aims: Recent research indicates that disruption of olfactory function after paediatric traumatic brain injury (pTBI) is common. Importantly, olfactory dysfunction (OD) is known to have significant functional implications in areas of health, safety and quality of life. Despite this longitudinal research investigating olfactory recovery and the functional impact of OD in pTBI is limited. This study aimed to track the recovery of olfactory function following pTBI and investigate predictors of early and late olfactory outcomes.

Method: The olfactory function of 37 children with TBI, aged 8-16 years, was assessed at $0-3,8$ and 18 months post-injury using the University of Pennsylvania Smell Identification Test. Data were collected on demographic and injury variables as well as the impact of OD on day-to-day function.

Results: Significant improvements in olfactory performance were evident over time in the group presenting with OD acutely. For the subgroup with the most severe OD only $16 \%$ showed recovery to normal olfactory function, with the remainder demonstrating ongoing olfactory impairment 18 months post-injury. While predictors of early and late olfactory outcomes varied, occipital site of impact was a significant predictor of later olfactory performance.

Conclusions: Our results suggest that while there is evidence of recovery of OD over time after pTBI, those children with more severe OD acutely are likely to have persisting OD. Given this limited recovery, understanding the functional implications of OD and implementation of appropriate 
management strategies is considered an important aspect of overall rehabilitation management of children with TBI.

Correspondence: Kathleen Bakker; kath.bakker@rch.org.au

\title{
Academic Outcomes from Traumatic Brain Injury in Very Early Childhood
}

\author{
Louise Crowe, ${ }^{1,2,3}$ Cathy Catroppa ${ }^{1,2,3,4}$ and Vicki Anderson 1,2,3 \\ ${ }^{1}$ Clinical Sciences, Murdoch Childrens Research Institute, Melbourne, Australia \\ ${ }^{2}$ School of Psychological Sciences, University of Melbourne, Melbourne, Australia \\ ${ }^{3}$ Psychology Department, Royal Children's Hospital, Melbourne, Australia \\ ${ }^{4}$ Department of Paediatrics, University of Melbourne, Melbourne, Australia
}

\begin{abstract}
$B$ ackground and aims: Traumatic brain injury (TBI) is a common injury in children under 3 years of age. A TBI in childhood can result in lowered IQ, and language, attention, and memory difficulties. In turn, academic function is impacted and poor academic ability has significant long-term consequences. The influence of injury variables as well related skills of language and working memory were investigated.

Method: The academic ability of 28 children with TBI, aged 7-10 years, was assessed at 6 years post-injury. Children were aged $0-3$ years at injury. A comparison group of healthy uninjured controls matched for demographic factors $(n=15)$ was included. This was a longitudinal study with children assessed at 3 and 6 years post-injury. Data were collected on demographic and injury variables. The role of injury variables, early language and working memory on academic outcomes was investigated.

Results: Children with a moderate to severe TBI had the lowest scores on a measure of reading, spelling and mathematics, with moderate to large effect sizes when compared to children with mild TBI or uninjured children. Predictors of outcome included injury variables (Glasgow Coma Score, loss of consciousness), language scores at 3 years post-injury, and performance on working memory tasks.

Conclusions: Children with moderate to severe TBI are at increased likelihood of difficulty with reading and mathematics. Academic skills are influenced by injury variables, early language and working memory skills. How children with TBI can be assisted to reach their academic potential will be discussed.
\end{abstract}

Correspondence: Louise Crowe; louise.crowe@mcri.edu.au

\section{Sleep Disturbances Following 20 Years of Childhood Traumatic Brain Injury}

Edith Botchway, ${ }^{1,2,3}$ Celia Godfrey, ${ }^{1,2,3}$ Vicki Anderson, ${ }^{1,2,3}$ Christian Nicholas ${ }^{3,4}$ and Cathy Catroppa ${ }^{1,2,3}$

${ }^{1}$ Child Neuropsychology Group, Murdoch Childrens Research Institute, Melbourne, Australia

${ }^{2}$ Royal Children's Hospital, Melbourne, Australia

${ }^{3}$ University of Melbourne, Melbourne, Australia

${ }^{4}$ Sleep Research Laboratory, Melbourne School of Psychological Sciences, University of Melbourne, Melbourne, Australia

$\mathcal{R}$ ackground and aims: Sleep disturbances are common after Traumatic 0 Brain Injury (TBI). Emerging evidence demonstrates that sleep disturbances can persist several years following TBI in childhood. However, the impact of childhood TBI on sleep beyond adolescence remains unknown. This study aims at investigating the prevalence, types, and predictors of sleep disturbances in adults with childhood TBI.

Method: Participants are part of an original cohort of 172 children with TBI (mild, moderate, and severe) and 35 healthy controls, recruited between 1993 
and 1997. Sleep was assessed subjectively using validated self-report sleep measures, and objectively using actigraphy.

Results: Preliminarily results from 14-days actigraphy recording of 7 adults with childhood TBI 5 control participants show a trend towards poor sleep in the TBI compared to the control group. Specifically, the TBI group seem to have a higher sleep duration, longer sleep onset latency, poorer sleep efficacy, and more wake after sleep onset; all with medium to large effect sizes.

Conclusions: These preliminary results indicate that childhood TBI can impact sleep in adulthood. Should these trends continue, these findings will provide a robust evidence base for the regular assessment, and treatment of sleep disturbances following TBI in childhood. This could further inspire the use of sleep interventions to improve sleep and related outcomes in this TBI population.

Correspondence: Edith Botchway; edith.botchway@mcri.edu.au

\title{
Fatigue Outcome from Child Brain Injury: Identifying those at Risk of Poor Outcome 1 Year Postinjury
}

\author{
Alison Crichton, ${ }^{1,2,3}$ Franz Babl, ${ }^{2,4,5}$ Ed Oakley, ${ }^{2,4,5}$ Mardee Greenham, ${ }^{2,3}$ Stephen \\ Hearps, ${ }^{2}$ Carmel Delzoppo, ${ }^{2,5}$ Miriam H. Beauchamp, ${ }^{6,7}$ Jamie Hutchison ${ }^{8}$ and Vicki \\ Anderson $2,3,5$ \\ ${ }^{7}$ Victorian Pediatric Rehabilitation Service, Monash Children's, Melbourne, Australia \\ ${ }^{2}$ Murdoch Childrens Research Institute, Melbourne, Australia \\ ${ }^{3}$ School of Psychological Sciences, University of Melbourne, Australia \\ ${ }^{4}$ Department of Pediatrics, University of Melbourne, Australia \\ ${ }^{5}$ Royal Children's Hospital, Melbourne, Australia \\ ${ }^{6}$ Department of Psychology, University of Montreal, Montreal, Canada \\ ${ }^{7}$ Research Center, Ste-Justine Hospital, Montreal, Canada \\ ${ }^{8}$ Neuroscience and Mental Health Research Program, Hospital for Sick Children Research Institute, \\ Toronto, Canada
}

$\boldsymbol{R}$ ackground and objectives: Fatigue is a common and persisting sympB tom after child TBI, yet longitudinal data is lacking. It is deleterious to quality of life, child functioning and participation in typical childhood activities school. The purpose of the present study was to examine whether injury severity and pre-and post-injury fatigue, demographic and psychological risk factors predict persistent fatigue at 1 year after injury.

Method: This longitudinal prospective study assessed parent rated multidimensional fatigue symptoms using the PedsQL Multidimensional Fatigue Scale (PedsQL MFS) in 104 children (2-18 years), 1 year postinjury across 3 participating hospitals. Participants presented with brain injuries (mild-severe). We collected data on demographics, symptoms (fatigue, sleep, pain) and functioning (adaptive and psychological functioning) reported retrospectively at recruitment. Injury data was collected via medical record review. Primary fatigue outcome was assessed using the PedsQL MFS questionnaire completed 1 year post-injury. Univariate and multivariate regressions were used to examine the impact of pre-injury variables and injury severity on fatigue.

Results: A linear regression model containing all variables that were significant at the univariate level (psychological status, sleep disturbance, sex) explained $35 \%$ of the variance in fatigue symptoms $(p<.001)$. Female sex and psychological symptoms (depression/anxiety) were significant predictors of fatigue after child brain injury. 
Conclusions: These findings suggest that multiple biological and psychological factors are important contributors to fatigue. Findings provide insight into those at risk of poor outcome, and potential avenues for early intervention to reduce the impact on functioning in day to day life.

Correspondence: Alison Crichton; ali.crichton@mcri.edu.au

\title{
Concurrent Session 19 - How to Session
}

\section{Scams and Brain Impairment: A Clinician's Treatment Recommendations and a Survivor's Perspective}

\author{
Kate R. Gould ${ }^{1,2}$ and Colin Brokenshire \\ ${ }^{1}$ Thinkfully, Melbourne, Australia \\ ${ }^{2}$ Monash Epworth Rehabilitation Research Centre, Monash University
}

\begin{abstract}
C ynopsis of session: Whilst the increase in global connectivity through the internet has resulted in overwhelmingly positive benefits, a disadvantage is the exponential rise of scams, which are rife in Australia and around the world. The Australian Competition and Consumer Commission reported \$81.6 million lost to scams in 2016. Scammers are becoming more sophisticated and well-resourced. Although (counter-intuitively) well-educated, computersavvy and financially literate individuals are the most common target victims; individuals with brain impairment are particularly vulnerable. There is an awareness of this additional risk for individuals with disability in regards to the availability of tips for scam prevention on the internet. However, to our knowledge, there are no recommendations to support clinician's treatment of individuals with or without brain impairment who have been scammed.

This 'How To' session will provide an overview of the research available regarding the psychology of scams and draw from more than two years of intervention directed towards understanding and recovering from a relationship scam in an individual with a brain injury. The session will include a presentation from the perspective of the scam survivor.
\end{abstract}

\section{Concurrent Session 20 - How to Session}

\section{Learn How to Tweet: Using Social Media for Research and Rehabilitation}

\author{
Melissa Brunner, ${ }^{1}$ Elizabeth Beadle ${ }^{2}$ and Rachael Rietdijk ${ }^{3}$ \\ ${ }^{7}$ Speech Pathology, Faculty of Education and Arts, University of Newcastle, Newcastle, Australia \\ ${ }^{2}$ School of Applied Psychology and Menzies Health Institute, Griffith University, Mt Gravatt, Australia \\ ${ }^{3}$ Speech Pathology, Faculty of Health Sciences, University of Sydney, Sydney, Australia
}

\footnotetext{
C ynopsis of session: This session will provide an overview of using social media professionally, academically, and clinically. It will include a practical component specifically addressing Twitter skill development. Attendees will have the added benefit of being able to connect and practice these skills after the session with support from the session facilitators and their peers whilst attending the conference.
} 


\title{
POSTERS
}

FRIDAY 2nd JUNE

\section{Attention, Executive Function and Connected Speech Deficits in Mild Stroke}

\author{
Megan S. Barker, Breanne Young and Gail A. Robinson \\ Neuropsychology Research Unit, School of Psychology, The University of Queensland, St Lucia, \\ Brisbane, Australia
}

\begin{abstract}
$\boldsymbol{R}$ ackground and aims: Prelinguistic conceptual preparation processes are 0 involved in the production of cohesive and coherent connected speech. Cohesion and coherence generally connect sentences with preceding ideas and the overall topic, and broader cognitive mechanisms may mediate these processes. This study investigated (1) whether stroke patients without aphasia exhibit coherence and cohesion impairments in connected speech, and (2) the role of attention and executive functions in the production of connected speech. Method: Eighteen stroke patients ( 8 right hemisphere stroke [RHS]; 6 left hemisphere stroke [LHS]) and 21 healthy controls completed two selfgenerated narrative tasks to elicit connected speech. A multi-level analysis of within and between-sentence processing ability was conducted.

Results: Cohesion and coherence impairments were found in the stroke group, particularly RHS patients, relative to controls. In the whole stroke group, better performance on the Hayling Test of executive function, which taps verbal initiation/suppression, was related to fewer propositional repetitions and global coherence errors. Better performance on attention tasks was related to fewer propositional repetitions, and decreased global coherence errors. In the RHS group, aspects of cohesive and coherent speech were associated with better performance on attention tasks. Better Hayling Test scores were related to more cohesive and coherent speech in RHS patients, and more coherent speech in LHS patients.

Conclusions: We have documented connected speech deficits in a heterogeneous stroke group without prominent aphasia. Our results suggest that broader cognitive processes may play a role in connected speech production at the early conceptual preparation stage.
\end{abstract}

Correspondence: Megan Barker; megan.barker@uqconnect.edu.au

\section{The Lived Experience of Cognitive Remediation for People with Schizophrenia: A Qualitative Comparison with an Active Control}

\author{
Shayden Bryce, ${ }^{1,2}$ Stuart Lee, ${ }^{1,2}$ Narelle Warren, ${ }^{1}$ Jennie Ponsford ${ }^{1,3}$ and Susan \\ Rossell ${ }^{2,4}$ \\ ${ }^{7}$ School of Psychological Sciences, Monash University, Clayton, VIC, Australia \\ ${ }^{2}$ Monash Alfred Psychiatry Research Centre, The Alfred and Monash University Central Clinical \\ School, Melbourne, VIC, Australia \\ ${ }^{3}$ Monash-Epworth Rehabilitation Research Centre, Richmond, VIC, Australia \\ ${ }^{4}$ Brain and Psychological Sciences Research Centre, Swinburne University of Technology, Hawthorn, \\ VIC, Australia
}

$\boldsymbol{R}$ ackground and aims: Cognitive remediation (CR) has emerged as a $B$ promising method of improving cognitive and psychosocial outcomes in people with schizophrenia. Nevertheless, the lived experience of participation, and how this differs from computer-game (CG) controls, has been seldom explored. The aim of this study was to qualitatively examine and compare the experience of participation in these group-based interventions. 
Method: Forty-two people with schizophrenia/schizoaffective disorder completed a qualitative survey with seven open-ended questions after completing 10 weeks of CR $(n=22)$ or CG $(n=20)$. Thematic analysis was used to identify codes and organize them into higher-order themes. Code frequency was recorded to facilitate descriptive comparisons between groups.

Results: Four overarching themes were identified: 1) Perceived benefits (e.g. Cognitive and functional); 2) Experience of group (e.g. Fun/enjoyable); 3) Operation of group (e.g. Challenging and supportive); and 4) Suggestions for improvement. Overall, participation was generally viewed positively and similar themes emerged in both groups. The CR participants, however, were more likely to report: improvements in memory (32\% vs 5\%), greater community/everyday participation ( $32 \%$ vs $5 \%$ ), having learned new skills ( $41 \%$ vs $5 \%$ ), being challenged by group activities (45\% vs $15 \%$ ) and requiring teamwork to succeed (23\% vs $10 \%)$.

Conclusions: CR and CG share many qualitative benefits including social and intrinsic improvements, and are typically experienced as fun and/or satisfying. CR may, however, provide participants with additional opportunities to learn new cognitive strategies, challenge themselves, and succeed as a team. These differences may foster improvements in self-efficacy; a construct infrequently examined in research trials.

Correspondence: Shayden Bryce; Shayden.Bryce@monash.edu

\section{A Transdisciplinary Approach to Brain Injury Rehabilitation}

Chris Catchpole

Hunter Brain Injury Service, Hunter New England Local Health District, Newcastle, Australia

$\boldsymbol{R}$ ackground and aims: Transdisciplinary clinical practice is not widely used in specialised services, however, it has the potential to increase efficiency through reducing duplication and fragmentation of care. This project was initiated in response to staff and patient feedback during 'rounding', where concerns were raised over clinical duplication. A review of the Hunter Brain Injury Service (HBIS) model of care highlighted key areas where unwanted duplication was occurring and this was confirmed by clinical audit. Occupational Therapy workload, when compared to the rest of the team, was high and their waiting time for therapy commencement was significantly higher than other disciplines.

Method: A shared care approach was established across all disciplines in HBIS, with defined roles and responsibilities for undertaking assessments. New assessments were designed for use across disciplines, with the focus on community access assessment, upper limb assessments and ADL functional assessments. The redesigned assessments were incorporated into local models of care and relevant clinical guidelines.

Results: Unwanted clinical duplication was reduced from 50\% to 0\% in upper limb assessment; Occupational Therapy waiting time reduced 25 days; and there was a $62 \%$ increased utilisation of rehabilitation assistants for functional assessment and retraining tasks.

Conclusions: The transdisciplinary nature of the new assessments encouraged mutual respect for each discipline, improved communication between clinicians and ultimately enhanced collaboration and teamwork. Implementation of the transdisciplinary approach has resulted in higher-value healthcare, through increased efficiency and increased clinician scope of practice.

Correspondence: Chris Catchpole; Christopher.Catchpole@hnehealth. nsw.gov.au 


\title{
Life Beyond Residential Aged Care: Revisiting Rehabilitation Potential to Facilitate Community Living Following Severe Brain Injury
}

\author{
Laura DeLacy, ${ }^{1,2}$ Danielle Sansonetti ${ }^{1,2}$ and Michelle Farquhar ${ }^{2}$ \\ ${ }^{1}$ Occupational Therapy Department, Alfred Health, Caulfield, Australia \\ ${ }^{2}$ Acquired Brain Injury Rehabilitation Centre, Alfred Health, Caulfield, Australia
}

$\boldsymbol{R}$ ackground and objectives: Recovery following severe brain injury can $\mathcal{B}$ be a lifelong process, with complex and often persisting cognitive, behavioural, and physical deficits increasing the risk of premature admission to residential aged care (RAC). The aim of this paper is to explore rehabilitation pathway of a single case, who presented to our service during the chronic phase post-brain injury. This paper will explore the role of a transitional living service (TLS) in facilitating community re-integration.

Method: Single, descriptive case study which outlines the consumer experience of rehabilitation services for a 57 year old male living in RAC. Semistructured interviews explored the resident and significant others' experiences of rehabilitation across a seven year timeframe. Qualitative data from interviewees were synthesised thematically, and routine outcome data (Care and Needs Scale, Community Integration Questionnaire, and FIM ${ }^{\mathrm{TM}}$ ) were extracted from the medical record.

Results: Findings suggest that a TLS program served as a stepping stone towards returning to independent living. They also highlighted the unique opportunity the TLS had provided to them, feeling that the opportunity for rehabilitation had been best facilitated by the TLS environment than any other setting across the continuum. Following the TLS program, the participant returned to live in his own home with carer support. Outcome measures taken during rehabilitation highlight the importance of a TLS program within an extended rehabilitation pathway.

Conclusions: The rehabilitation pathway following severe brain injury is not linear and TLS programs offer a unique opportunity to explore the rehabilitation potential for individuals living in RAC.

Correspondence: Danielle Sansonetti; D.Sansonetti@alfred.org.au

\section{Preliminary Feasibility and Efficacy Evaluation of a Group-Based Cognitive Intervention Program for Asian Patients with Mild Cognitive Impairment}

YanHong Dong, ${ }^{1,2}$ Kim A. Granland, ${ }^{2,4}$ Claire Thompson, ${ }^{4}$ Chia Hui Khoo, ${ }^{3}$ Esther Yee Shuang Wan ${ }^{1}$ and Rathi Mahendran ${ }^{1}$

${ }^{1}$ Psychological Medicine, National University Hospital, Singapore

${ }^{2}$ Department of Medicine (Neurology Division), Yong Loo Lin School of Medicine, National University of Singapore, Singapore

${ }^{3}$ Department of Medicine (Neurology Division), National University Hospital, Singapore

${ }^{4}$ James Cook University, Singapore Campus, Singapore

$\boldsymbol{R}$ ackground and aims: An innovative cognitive intervention group pro3 gram is required for patients with Mild Cognitive Impairment (MCI) due to the lack of pharmaceutical treatments. The "Train Your Brain" (TYB) cognitive intervention group program customized for Singaporean patients to remediate cognitive impairments in patients with MCI. In this pilot study, we aimed to evaluate the feasibility and efficacy of TYB program.

Method: 17 MCI patients aged $\geq 50$ years old were recruited from the memory clinic at National University Hospital, Singapore. A randomized control trial with cross-over design is adopted. The TYB group will attend a 9-session group program on brain health and cognitive training. The control group will 
be waitlisted initially and crossed over to become an intervention group. All patients received pre/post measures of cognition [Mini-Mental State Exam and Montreal Cognitive Assessment (MoCA) as well as a formal neurocognitive battery], psychological wellbeing, memory self-efficacy and daily functioning. Two samples $t$-tests will be employed to compare change in measures between groups.

Results: Approximately $2 / 3$ of the patients could complete the whole TYB program. They have provided positive feedback such as improved self-efficacy and less anxiety pertaining to memory problems. Despite the small sample size, significant improvement in MoCA has been observed (pre-program: 22.5 \pm 4.4 vs post-program: $23.7 \pm 4.0, p=.04$ ).

Conclusions: Our pilot study has demonstrated preliminary evidence for feasibility and efficacy of TYB program as a culturally and linguistically appropriate for MCI patients in Singapore. Data collection is ongoing. The change scores in formal neuropsychological tests and psychological wellbeing will be discussed.

Correspondence: YanHong Dong; catherine_dong@nuhs.edu.sg

\title{
Preliminary Evaluation of a Multi-Disciplinary Concussion Clinic in Melbourne
}

\author{
Harvey Jones, ${ }^{1}$ Jo Sherry, ${ }^{1}$ Megan Hamilton, ${ }^{1}$ Bianca Fedele, ${ }^{1,2}$ Michelle \\ Morandin, ${ }^{1}$ Rose Acher $^{1}$ and John Olver ${ }^{1,2}$ \\ ${ }^{1}$ Epworth Concussion Clinic, Epworth Rehabilitation Hawthorn, Epworth HealthCare, Melbourne, \\ Australia \\ ${ }^{2}$ Epworth Monash Rehabilitation Medicine Unit (EMReM), Epworth HealthCare/Monash University, \\ Australia
}

$R$ ackground and aims: A substantial body of literature has focussed on the acute diagnosis and management of concussion in addition to risk factors that might predict post-concussion syndrome (PCS); persisting concussive symptoms over a prolonged period (longer than 7-10 days). Conversely, little research has examined the treatment and management of PCS clinically. This is despite evidence that between $15-25 \%$ of individuals with concussion go on to develop PCS and that, according to a recent review, routine preventative strategies, such as early education, have a marginal impact on symptoms. In 2016, Epworth HealthCare launched a multi-disciplinary concussion clinic.

Method: The clinic is overseen by a Rehabilitation Physician and includes a Neuropsychologist and Physiotherapist. Patients are administered the PostConcussion Symptom Checklist (an indication of concussion symptom presence), the Sharpened Rhomberg Balance Test (a static test of postural control) and various follow-up measures to track subsequent progress in terms of mood and function. Each intervention is individually tailored and includes education, skills training (mindfulness, behaviour change, tolerance of some symptoms when increasing activity, etc.) and exercises.

Results: Initial pilot data indicates that on average, the patients have been symptomatic for 169.05 days $(S D=362.17)$ at the time of admission. Preliminary data is presented suggesting that the program lowers symptom severity on the post-concussion symptom checklist. Other data associated with mood, functional outcome, demographic data and injury profile are also explored.

Conclusions: Whilst the initial findings of this evaluation are encouraging, the program requires study over a longer period and further evaluation in a clinical trial.

Correspondence: Harvey Jones; harveyj42@gmail.com 


\title{
Do We Need to Talk About Sex? - Sexuality After Stroke
}

Jane Khoo, ${ }^{1,2}$ Susie Leech, ${ }^{1,3}$ Katy Meltzer ${ }^{1,2}$ and Emma Schneider ${ }^{1,4}$

${ }^{1}$ Neurological Rehabilitation Program, Caulfield Hospital, Alfred Health

${ }^{2}$ Psychology Services, Caulfield Hospital, Alfred Health

${ }^{3}$ Social Work Services, Caulfield Hospital, Alfred Health

${ }^{4}$ Occupational Therapy Department, Caulfield Hospital, Alfred Health

$\boldsymbol{R}$ ackground and aims: This project aimed to improve the Neurological B Rehabilitation unit's approach to discussing sexuality with patients and partners.

The National Stroke Foundation Clinical Guidelines for Stroke Management, Guideline 8.5, states patients and their partners should be provided with written information addressing sexuality after stroke, and the opportunity to discuss sex and sexual issues with an appropriate health professional. This research study conducted a needs analysis and explored how the unit might achieve a more consistent approach in addressing sexuality.

Method: Clinicians completed a survey to explore their knowledge and attitude regarding sexuality post-stroke. Stroke patients were also interviewed to provide feedback on the NSF sexuality factsheet and our unit's current processes.

Results: All surveyed clinicians recognised that changes to sexuality may be an issue after stroke. The majority (95\%) believed that providing clients with information about changes to sexuality after stroke is important, but $60 \%$ did not feeling confident in their ability to provide this information. There was no consensus on a specific discipline to be solely responsible for addressing this topic with patients.

All stroke patients interviewed expressed an absolute need for sexuality to be addressed during rehabilitation.

Conclusions: This needs analysis confirmed that both staff and patients feel that sexuality should be discussed during a stroke patient's rehabilitation. Staff members appear to lack the confidence to raise and/or discuss the topic with patients. Results substantiate the need for staff education seminars on sexuality post stroke. An action plan will be designed to facilitate improvements in processes and practice.

Correspondence: Jane Khoo; j.khoo@alfred.org.au

\section{Telehealth Delivery of Memory Rehabilitation Following Stroke}

\author{
David Lawson, ${ }^{1,2}$ Rene Stolwyk, ${ }^{1,2}$ Jennie Ponsford ${ }^{1,2}$ and Dana Wong ${ }^{1,2}$ \\ ${ }^{1}$ School of Psychological Sciences \& Monash Institute of Cognitive \& Clinical Neurosciences, Monash \\ University, Melbourne, Australia \\ ${ }^{2}$ Monash-Epworth Rehabilitation Research Centre, Melbourne, Australia
}

$\boldsymbol{B}$ ackground and aims: Around half of stroke survivors experience memory difficulties, affecting independence, work capacity, and quality of life. Memory rehabilitation programs significantly improve memory function, however many face barriers to access, including geographic location, mobility restrictions, and cost. Telehealth provision holds potential to overcome these, however evidence is lacking that this delivery mode results in similar positive outcomes. This pilot study aimed to establish feasibility and potential effectiveness of an internet-based delivery mechanism for a rehabilitation program after stroke.

Method: Participants $(N=5)$ engaged in a 6-week memory training program, delivered individually using an internet-based videoconferencing program. 
Utilising a single case experimental design, memory function was measured across baseline, intervention and follow-up phases using the Everyday Memory Questionnaire (EMQ-R), Comprehensive Assessment of Prospective Memory (CAPM), and a diary of ratings for overall memory functioning. Technology failure rate, treatment dropout rate, and participant satisfaction levels were also examined.

Results: Significant positive change in memory function was observed for four of the five cases using the EMQ-R, with mixed results using the CAPM and functional memory ratings. Low technology failure rate and treatment dropout were observed, alongside high participant satisfaction levels.

Conclusions: The results suggest that a telehealth-based intervention may be a feasible and effective delivery method for memory rehabilitation following stroke.

Correspondence: David Lawson; david.lawson@monash.edu

\title{
The Experience of Recreational Camp for Families with a Child or Young Person with Acquired Brain Injury (ABI)
}

\author{
Katrina Luzinat, ${ }^{1}$ Amelia Hicks, ${ }^{1}$ Penelope Analytis, ${ }^{1}$ Narelle Warren ${ }^{2}$ and Jennie \\ Ponsford $^{1}$ \\ ${ }^{1}$ Monash Epworth Rehabilitation Research Centre, Monash University, Melbourne, Australia \\ ${ }^{2}$ School of Social Sciences, Monash University, Melbourne, Australia
}

$\boldsymbol{B}$ ackground and objectives: Paediatric acquired brain injury (ABI) can profoundly affect the lives of children and their families. As the family environment influences child recovery, it is important to support families. The Heads Together recreational camp (HTC) is a community support service run for families with a child or young person with ABI. This study sought to explore parents' perspectives on participating in HTC and how participation had impacted them and their family.

Method: Semi-structured interviews were conducted with 11 parents $\left(M_{\mathrm{age}}=\right.$ $44, S D_{\text {age }}=4.96 ; 9$ mothers, 2 fathers) who had attended HTC. Transcripts were coded and analysed using an inductive approach. Member checking was conducted.

Results: Using thematic analysis, six themes were identified; $A B I$ and the family; Discomfort and Apprehension; Connections and Community; Fun, Relaxation and Respite; Hope and Perspective; and Family Functioning. Parents experienced initial feelings of apprehension at camp which attenuated as connections were developed. Camp provided an accepting and supportive community, where parents felt less alone and could share advice, in a fun and relaxing environment.

Parents felt that camp gave them hope and perspective and led to improvements in aspects of family functioning.

Conclusions: Peer support provided through recreational camps may be used to mitigate some of the stressors experienced by children with $\mathrm{ABI}$ and their families. Healthcare providers may encourage participation in camps or other programs that offer opportunities to share family experiences of ABI.

Correspondence: Amelia Hicks; Amelia.hicks@monash.edu 


\title{
The Untapped Potential for Engaging Friends of Young People with TBI in Community Rehabilitation: A Unique Case Report
}

\author{
Sue Wright $^{1}$ and Clare Morgan ${ }^{2}$ \\ Acquired Brain Injury Outreach Service, Brisbane, Australia
}

\begin{abstract}
$B$ ackground: Social isolation is common among young people following traumatic brain injury (TBI) with family becoming the principal support rather than peers (Kosciulek, 1997). Rehabilitation professionals often fail to engage friends in rehabilitation. A case report of 'Ben' who sustained a severe TBI with complex impairments will be discussed. Following an escalation in Ben's behavioural challenges due to social isolation and an emerging awareness of the differences between Ben and his friends, the family were at risk of breakdown. A treatment model involving friends in Ben's rehabilitation was used to circumvent these challenges.

Method: A goal-directed community rehabilitation program using a collaborative practitioner approach was implemented. A Circle of Support (Snow \& Forest, 1987) which has been used extensively in clinical groups to improve community participation (Rowlands, 2001) was also implemented. This Circle included Ben, his family and friends as way of empowering those involved to support Ben.

Results: The unique finding was the emergence of Ben's long-term friend as Circle of Support leader. This model gave Ben's friend a role in his rehabilitation which had not previously been offered. The involvement of his friend decreased Ben's social isolation and reduced his parent's carer burden.

Conclusion: The young male TBI population are a vulnerable cohort making them susceptible to social isolation and exposing families to breakdown. The Circles of Support approach can become significantly more powerful when driven by peers. Friends can play significant roles in rehabilitation, but need to be given opportunity and direction to be involved in rehabilitation.
\end{abstract}

Correspondence: Sue Wright; suzanne.wright@health.qld.gov.au

\section{Is Structural Neuropathology Associated with Cognitive Functioning Following Mild Traumatic Brain Injury?}

Lucy Oehr ${ }^{1}$ and Jacqueline Anderson ${ }^{1,2}$

${ }^{7}$ Melbourne School of Psychological Sciences, The University of Melbourne, Melbourne, Victoria, Australia

${ }^{2}$ Psychology Department, The Alfred hospital, Melbourne, Victoria, Australia

R ackground and objectives: Although psychiatric/psychological factors $\mathcal{B}$ are known to increase the risk of poor cognitive recovery after mild traumatic brain injury (mTBI), clinicians continue to have difficulty predicting individual recovery trajectories. This is unsurprising given that standard clinical measures of neuropathological severity are poor predictors of cognitive recovery after $\mathrm{mTBI}$. Traditional structural neuroimaging techniques have also been unable to identify an in vivo relationship between structural neuropathology and cognitive dysfunction after mTBI, limiting their clinical relevance with respect to predicting cognitive recovery. A recently developed structural neuroimaging technique [diffusion tensor imaging (DTI)] may be capable of measuring the in vivo relationship between structural pathology and cognitive function after mTBI. We undertook a systematic review and meta-analysis to determine whether greater white matter neuropathology, as measured by DTI, is associated with poorer cognitive performance after mTBI. 
Methods: Of the 248 original papers retrieved, 8 were included in the metaanalysis; the final sample included $220 \mathrm{mTBI}$ patients.

Results: A significant linear association was found between DTI metrics and performance on measures of attention $(p<.001)$, memory $(p<.001)$, and executive function $(p<.05)$.

Conclusions: The results provide the first clear evidence of an in vivo relationship between structural neuropathology and cognition following mTBI, with more severe white matter neuropathology associated with poorer performance on measures of attention, memory, and executive function. These findings demonstrate clearly that we are now able to directly investigate the relationship between structural neuropathology and cognitive recovery after mTBI. There are significant clinical consequences of this finding, which will be considered.

Correspondence: Jacqueline Anderson; jfande@unimelb.edu.au

\title{
Working on Work: A Scoping Review of Work Interventions Provided to Adults with Brain Injury During Hospitalisation
}

\author{
Sophie O'Keefe, ${ }^{1}$ Natasha Lannin, ${ }^{1,2}$ Mandy Stanley ${ }^{3,4}$ and Kerry Adam ${ }^{5}$ \\ ${ }^{1}$ La Trobe University, Melbourne, Australia \\ ${ }^{2}$ Alfred Health, Melbourne, Australia \\ ${ }^{3}$ University of South Australia, Adelaide, Australia \\ ${ }^{4}$ Griffiths University, Brisbane, Australia \\ ${ }^{5}$ Australian Catholic University, Brisbane, Australia
}

\begin{abstract}
$B$ ackground and aims: Return to work is a common goal in brain injury rehabilitation. The focus in acute rehabilitation appears to be on restoring independence in activities within the home, perhaps at the expense of work interventions. Further, clinicians report low confidence to implement work interventions, suggesting a gap in our understanding of the complex field of return to work. The aim of this scoping review was to explore published evidence on the experience of and engagement in return to work rehabilitation during the inpatient phase of brain injury recovery.

Method: A systematic search of four databases was conducted to identify studies that investigated the effectiveness of hospital based work interventions, the consumer experience, and/or the clinician perspective of commencing work interventions during inpatient rehabilitation. Databases were searched from their inception, and the end date was December 2016. Thematic analysis of the findings was completed to synthesise findings.

Results: Systematic searches yielded 31 papers $(n=16$ quantitative, $n=8$ qualitative studies, $n=7$ other). Findings identified the importance of a multiphase intervention approach for early work rehabilitation, that consisted of; (1) developing confidence and belief in future work capacity (2) development of work habits and routines, (3) assessment and training of work skills, and (4) rehabilitation program structured to support return to work.

Conclusion: This review supports the value of commencing work rehabilitation early in recovery after brain injury. Findings provide specific guidance for the commencement of work-related intervention during the inpatient phase of rehabilitation.
\end{abstract}

Correspondence: Sophie O’Keefe; s.okeefe2@latrobe.edu.au 


\title{
When All the Doors Close: Complex Discharge Planning for Individuals with Severe Cognitive-Behavioural Impairments
}

\author{
Danielle Sansonetti, Jacinta Sadler and Mithu Palit \\ Acquired Brain Injury Rehabilitation Centre, Alfred Health, Caulfield, Australia
}

\begin{abstract}
$B$ ackground and objectives: Cognitive impairment combined with behaviours of concern can have a considerable impact on discharge destination options available to individuals following severe brain injury. Many residential aged care facilities are not able to provide a specialised and consistent approach to managing behaviours of concern that would afford sustainability of these living arrangements, leading to a lack of suitable accommodation options for individuals who are unable to be supported in their own home setting. This paper aims to describe the complexities and challenges that exist throughout the discharge planning process for individuals with behaviours of concern and cognitive impairments following severe acquired brain injury.

Method: This descriptive case study will outline the ethical considerations and process of exploring least restrictive discharge options for a 49 year old man with severe cognitive-behavioural impairment across a 12-month rehabilitation timeframe. Semi-structured interviews explored the treating rehabilitation team's experience throughout the discharge planning process, with qualitative interview data synthesised thematically.

Results: Themes and associated best available evidence will be presented including: patient factors, patient and family engagement, innovations in cognitive-behavioural interventions to facilitate discharge planning, risk management, ethical challenges, and accommodation sustainability. Enablers and barriers to discharge planning for patients with severe lack of awareness of deficits will also be explored.

Conclusions: Discharge planning is a complex process that requires an integrated team approach that includes the patient and significant others as central in this process. Innovative approaches are required to meet patient and significant others' aims for suitable community living.
\end{abstract}

Correspondence: Danielle Sansonetti; D.Sansonetti@alfred.org.au

\section{Evaluation of an Inpatient Yoga Program within a Brain Injury Rehabilitation Unit}

\author{
Rebecca Seeney, ${ }^{1}$ Emma Johnson, ${ }^{1}$ Janelle Griffin, ${ }^{1}$ Josephine Auster ${ }^{1}$ and Jenny \\ Fleming ${ }^{1,2,3}$ \\ ${ }^{1}$ Occupational Therapy Department, Princess Alexandra Hospital, Brisbane, Australia \\ ${ }^{2}$ School of Health and Rehabilitation Sciences, The University of Queensland, Brisbane, Australia \\ ${ }^{3}$ Centre for Functioning and Health Research, Metro South Health Services District, Brisbane, Aus- \\ tralia
}

$\boldsymbol{R}$ ackground and aims: Leisure participation provides a valuable platform for people to engage in meaningful, goal directed activity. An acquired brain injury $(\mathrm{ABI})$ may disrupt participation in many occupations, including leisure activities. Patient with ABI have reported a perceived lack of stimulating or meaningful activity whilst in rehabilitation. This study aims to explore patients' experiences and perceptions of the current yoga program within a brain injury rehabilitation unit and to evaluate the effectiveness of the yoga program on participants' relaxation and wellbeing before and after yoga participation.

Method: Participants are currently being recruited after volunteering to participate in a weekly yoga class. Participants complete a questionnaire at the beginning and end of each yoga class and are offered participation in a 
semi-structured interview. Quantitative data will be analysed using repeated measures t-tests. Qualitative interview data will be transcribed and the content analysed thematically using a Framework method.

Results: Preliminary qualitative data indicate a high level of satisfaction with participation in yoga. Participants report experiencing personal benefits including improved relaxation and increased knowledge of stretching and breathing techniques. Participants also report yoga positively influences other daily occupations (for example, sleep). They find yoga beneficial in providing a means of increasing activity participation whilst in inpatient rehabilitation.

Conclusions: Preliminary findings positively support both individual benefits of yoga and the program as a means of providing opportunity for increased activity participation.

Correspondence: Rebecca Seeney; rebecca.seeney@health.qld.gov.au

\title{
Teleneuropsychology Rehabilitation Services for Regional Inpatients with Stroke: Development and Evaluation of a Novel Pilot Program
}

\author{
Renerus J. Stolwyk, ${ }^{1,2}$ Lauren Arthurson, ${ }^{3}$ Joosup $\mathrm{Kim}^{4}$ and Dominique A. \\ Cadilhac $^{4}$ \\ ${ }^{1}$ Monash Institute of Cognitive and Clinical Neurosciences, School of Psychological Sciences, \\ Monash University, Melbourne, Australia \\ ${ }^{2}$ Monash-Epworth Rehabilitation Research Centre, Melbourne, Australia \\ ${ }^{3}$ Echuca Regional Health, Victoria, Australia \\ ${ }^{4}$ Stroke and Ageing Research, School of Clinical Sciences, Monash University
}

$\boldsymbol{B}$ ackground: Over two-thirds of survivors of stroke exhibit cognitive im$B$ pairment and/or mood disturbance. Unfortunately, access to neuropsychology services in remote and regional settings is limited. The aim of this research program is to develop and evaluate a new teleneuropsychology rehabilitation service for a regional Australian hospital.

Method: A pilot model of neuropsychological assessment and rehabilitation service provision for regional areas is under development. Currently, this clinical service is provided to the Echuca Regional Health inpatient rehabilitation unit by an experienced clinical neuropsychologist based at a Monash University clinic located in metropolitan Melbourne. The service includes comprehensive assessment of patient cognition, mood and behaviour in addition to provision of cognitive rehabilitation and psychological interventions. Education sessions and consultations are also provided to medical, nursing and allied health clinicians to assist them to manage patient neuropsychological impairments. A range of telehealth information communication technologies are utilised, with Zoom $^{\mathrm{TM}}$ the primary software program used.

Results and conclusion: Within the first 3 months of service implementation, six team education sessions, five neuropsychological assessments, and three team consultations for patient management have been provided. Pilot service evaluation data will be presented including proportion of patients receiving cognitive and mood assessments and therapy, associated waiting times, number of neuropsychological rehabilitation goals set and patient satisfaction. A cost description analysis is also planned to describe the potential economic benefits of this service, compared to other service options in order to support a business case for ongoing application of this new model of care.

Correspondence: Renerus J. Stolwyk; rene.stolwyk@monash.edu 


\title{
Behaviour Management - Can We Do It? Developing a Competency Framework for Behaviour Management in a Subacute Setting
}

\author{
Jennifer Todd, Kerrie Walters, Lynda Katona and Luke Delaney \\ Psychology Department, Caulfield Hospital, Alfred Health Melbourne, Australia
}

\begin{abstract}
$B$ ackground and aims: Behaviour issues such as aggression, inappropriate social and sexual behaviour are common in rehabilitation and aged care settings. While some psychologists have extensive experience in behaviour management this is an area of psychology that is frequently overshadowed by assessment and counselling. The Behaviour Project at Caulfield Hospital, Melbourne was developed to address this service gap by developing a competency framework in behaviour management for all psychologists. In addition an Advanced Practice role was developed to support staff. This project aimed to increase staff experience in writing plans, develop consistent practices and develop a clear framework for education of new staff.

Method: A competency framework was established outlining the core components in completing behaviour management plans, key training resources and assessment of skills. Training resources were made available including: workshops, reading, peer supervision and mentoring.

Results: Staff increased the number of formal behaviour plans completed and reported increased confidence in completing plans. Formal systems including: behaviour plan templates, behaviour meetings, assessment and education of new staff were established.

Conclusions: Behaviour management plans have been able to be established as a regular part of psychological services delivered. Challenges in establishing this change are discussed as well as broader issues in developing support for staff in the area of behaviour management.
\end{abstract}

Correspondence: Jennifer Todd; J.Todd@ alfred.org.au

\section{The Importance of Post-Acute Rehabilitation: A Case Study}

Janet Wagland, ${ }^{1}$ Elly Williams ${ }^{1}$ and Angelita Martini ${ }^{2}$

${ }^{1}$ Brightwater Care Group, Perth, Australia

${ }^{2}$ University of Western Australia

$\boldsymbol{B}$ ackground and aims: Following an acquired brain injury (ABI) a number $\mathcal{B}$ of people with complex rehabilitation needs do not receive the postacute rehabilitation services needed to make functional improvements. It is not uncommon for people with complex disability following ABI to reside in care facilities with the purpose of managing care needs as opposed to receiving rehabilitation. This case study describes the improvements made by a 53 year old individual after moving from an aged care facility to a post-acute rehabilitation facility, 3.5 years post $\mathrm{ABI}$, and highlights the importance of targeted rehabilitation.

Method: Routinely collected client outcomes (Mayo-Portland Adaptability Inventroy-4 (MPAI-4)) were analysed for the 53 year old individual, for ability, adjustment and participation on admission and at 3 monthly review. Comparisons of outcomes were made from admission to 2.5 years post admission.

Results: On admission to the service the client had moderate to severe limitations $($ TScore $=57)$, reducing to mild to moderate limitations $($ TScore $=46$ ) 2.5 years post admission. Ability reduced from mild to moderate limitations (TScore $=48)$ to mild limitations $($ TScore $=37$ ) and adjustment reduced from severe limitations $($ TScore $=60)$ to moderate to severe limitations. Participation 
reduced from severe limitations $($ TScore $=69)$ to mild to moderate limitations (TScore $=47$ ).

Conclusions: Improvements and functional gains can be made by individuals with ABI a number of years post injury when admitted to a post-acute rehabilitation service.

Correspondence: Elly Williams; elly.williams@brightwatergroup.com

\title{
The Well-Being of Caregivers of People with Acquired Brain Injury during Transition from Hospital to Home
}

\author{
Nicole Weir, ${ }^{1}$ Jennifer Fleming, ${ }^{1,2,3}$ Annalisa Conradie ${ }^{2}$ and Emily Nalder $^{4}$ \\ ${ }^{1}$ Occupational Therapy Department, Princess Alexandra Hospital, Brisbane, Australia \\ ${ }^{2}$ The University of Queensland, School of Health and Rehabilitation Sciences, Brisbane, Australia \\ ${ }^{3}$ Centre for Functioning and Health Research, Metro South Hospital and Health Service, Brisbane, \\ Australia \\ ${ }^{4}$ University of Toronto, Department of Occupational Science and Occupational Therapy \& March of \\ Dimes Canada, Toronto
}

\begin{abstract}
$\boldsymbol{R}$ ackground and objectives: Caregivers of people with acquired brain injury (ABI) may experience reduced wellbeing post-discharge. The study aimed to describe patterns of well-being for caregivers of individuals with ABI during the first 6 months following discharge, and to identify individual factors and sentinel events that predict caregiver well-being at 6 months.

Method: This prospective longitudinal cohort study collected data from 133 caregivers pre-discharge, and at 1, 3 and 6 month follow-ups using the Depression Anxiety and Stress Scales (DASS), EuroQol health questionnaire (EQ-5D), and the Carer Strain Index (CSI). Potential predictors of caregiver wellbeing included the level of disability of the person with ABI at discharge and sentinel events in family life in the first 6 months post-discharge. Caregiver well-being patterns were profiled descriptively and multiple regressions were used to determine predictors of caregiver well-being at 6 months postdischarge.

Results: Depression, anxiety, stress, and strain in caregivers tended to increase 3 months after discharge, with $33 \%$ of participants displaying extremely severe symptoms of depression, anxiety and stress at 6 months. Higher level of disability, and inability to return to work for the person with ABI and relationship breakdown were significant predictors of the final caregiver DASS score.

Conclusions: Generally caregiver well-being improved over time for the majority of caregivers. However, depression, anxiety and stress symptoms were a severe problem for a third of caregivers by 6 months. Identification of predictors of reduced caregiver well-being may enable clinicians to target those families at risk.
\end{abstract}

Correspondence: Nicole Weir; Nicole.weir@health.qld.gov.au

\section{Implementing the Physical Abilities and Mobility Scale (PAMS): A Pilot Study}

Meagan White ${ }^{1}$ and Noula Gibson ${ }^{1,2}$

${ }^{1}$ Physiotherapy Department, Princess Margaret Hospital for Children, Perth, Western Australia

${ }^{2}$ School of Physiotherapy and Exercise Science, Curtin University, Perth, Western Australia

$R$ ackground and objectives: Few objective outcome measurement tools exist for use in an acute paediatric inpatient setting to assess change in physical functioning following an acquired brain injury. The PAMS was developed at the Kennedy Krieger Institute and is a 20 -item quantitative scale. 
Our primary objective was to establish the clinical utility of the PAMS in an Australian paediatric healthcare setting.

Method: This was a pragmatic pilot study delivered as part of a quality improvement activity. Three children were assessed using the PAMS by the senior neurological physiotherapist. This was concurrently observed and assessed by a second senior physiotherapist and video-recorded. Two additional physiotherapists completed the PAMS assessment for each child from the video-recording. Parents completed a short questionnaire to assess their perceptions of the assessment process. Therapists provided written feedback on patient tolerance, carer engagement and logistical challenges.

Results: Average time to complete the PAMS was 23 minutes. Both the live and video assessments resulted in scores within 2 points of each other, indicating good reliability in scoring. Parents and therapists reported no concerns with assessment process, task difficulty, or assessment length, indicating good acceptance of the tool. Therapist's perceived the PAMS to be easy to use, with limited pre-requisite knowledge or training required compared to other tools. In all three cases, the PAMS facilitated communication regarding physical abilities and functional performance between the therapists, family and medical staff.

Conclusions: This study demonstrated strong clinical utility for the PAMS tool within an Australian healthcare setting.

Correspondence: Meagan White; Meagan.white@health.wa.gov.au

\section{SATURDAY 3rd JUNE}

\section{Assessment Guideline Implementation for a Comatose Patient}

Zoe Adams, ${ }^{1}$ Rhian Hurley, ${ }^{1}$ Michelle Farquhar, ${ }^{1}$ Eloise Thompson ${ }^{1,2}$ and Natasha Lannin ${ }^{1,3}$

${ }^{1}$ Occupational Therapy Department, Alfred Health, Melbourne, Australia

${ }^{2}$ Occupational Therapy Department, Melbourne Health, Melbourne, Australia

${ }^{3}$ La Trobe University, Melbourne, Australia

$\boldsymbol{R}$ ackground and aims: Standardised assessments for the person in a coma can contribute to planning, implementation and evaluation of care. This study aimed to develop, implement and evaluate a coma assessment protocol within an inpatient trauma service.

Method: An assessment protocol for using the Coma Recovery Scale-Revised (CRS-R) to monitor coma emergence on the acute neurosurgical and trauma wards was developed. Therapists received training following by implementation and evaluation using a before-and-after audit of CRS-R administration. Files were audited at baseline and 6-months post-implementation for adults admitted with a GCS $\leq 8$ after brain injury. Clinical utility surveys were completed by occupational therapists who administered the CRS-R.

Results: Our protocol improved use of the CRS-R from $0 \%$ to $80 \%(n=4)$ of eligible patients $(n=5$; total population $n=82)$. CRS-R was not completed in patients who were palliated $(n=18)$. Also affecting completion rates were the medical stability of patients or early transfer to rehabilitation. Staff reported challenges to implementation including familiarity with the assessment, convenience of the kit, difficulties completing alone, medical stability, patient availability and competing priorities.

Conclusions: Using an implementation framework increased the use of a standardised assessment to monitor coma emergence. Implementation took 
6-months suggesting that time is needed to change assessment behaviours in occupational therapists.

Correspondence: Zoe Adams; z.adams@alfred.org.au

\title{
Now You See Me: An Intervention to Target Visual Word Recognition Difficulties in Dyslexia
}

\author{
Emma Ashcroft and Carolyn Wilshire \\ School of Psychology, Victoria University of Wellington, Wellington, New Zealand
}

\begin{abstract}
$\boldsymbol{R}$ ackground and aims: Many reading-delayed children exhibit phonological impairments (PIs), characterised by difficulties reading novel words and poor performance on auditory phonological awareness tasks. For these children, interventions focusing on grapheme-phoneme correspondences (GPCs) appear to be effective. However some children do not fit this profile, with accurate word reading and good phonological awareness, they instead display critically slow word reading, and perform poorly on visual letter report tasks. This whole-word impairment (WWI) profile suggests a difficulty with parallel visual letter processing, which is essential whole word reading. These children may respond better to interventions that focus on whole-word recognition. In this study, we pilot such an intervention.
\end{abstract}

Method: Three reading delayed adolescents- two PI cases and one WWI casecompleted two different interventions. One was a computerised version of the typical GPC training. The other was a whole-word training that emphasised rapid visual recognition and semantic processing of whole, familiar words.

Results: The two PI cases read treated words more accurately and more rapidly following GPC training, but not following whole-word training. The WWI case responded positively to both interventions, but improvement was greater with whole-word training.

Conclusions: This pilot study suggests that tailoring interventions according to a child's cognitive profile may enhance their effectiveness. A fuller investigation with a larger sample size is warranted.

Correspondence: Emma Ashcroft; emma.ashcroft@ vuw.ac.nz

\section{Autobiographical Memory in Huntington's Disease}

Anna Carmichael and Julie Stout

School of Psychological Sciences, Monash University, Melbourne, Australia

$\boldsymbol{R}$ ackground and aims: Huntington's disease (HD) is a progressive autosomal-dominant neurodegenerative disorder characterised by motor, psychiatric and cognitive dysfunction. Cognitive decline in HD has been found to precede the hallmark motor symptoms of HD by an estimated ten years. To our knowledge, there has been no research to date examining autobiographical memory in HD, or the integrity of recent and remote autobiographical memory recall in the disease. The current study presents preliminary findings, whereby we compared episodic and semantic autobiographical memory recall in HD with healthy controls across five different life periods.

Method: Twenty-four participants with HD and 24 age-matched healthy controls (free from neurological disorders) were administered a semi-structured autobiographical memory interview (Autobiographical Interview).

Results: Participants with HD favoured the recall of semantic autobiographical memories (general knowledge details not connected to time or place), whereas age-matched controls were biased towards recalling episodic autobiographical 
memories (happenings, locations, emotions, perceptions from a specific time and place).

Conclusions: Preliminary findings suggest a deficit in episodic autobiographical memory recall in $\mathrm{HD}$, consistent with past literature that has found deficits in non-autobiographical episodic memory recall in both pre-symptomatic and symptomatic HD.

Correspondence: Anna Carmichael; anna.carmichael@monash.edu

\title{
"You do sort of panic for a moment, do you have the time?": Healthcare Professionals' Views on Talking to a Patient with Aphasia
}

\author{
Marcella Carragher, ${ }^{1}$ Robyn O'Halloran, ${ }^{1}$ Hillary Johnson, ${ }^{1}$ Nicholas Taylor ${ }^{2}$ and \\ Miranda Rose ${ }^{1}$ \\ ${ }^{1}$ Dept Community \& Clinical Allied Health, La Trobe University, Melbourne, Australia \\ ${ }^{2}$ Department of Rehabilitation, Nutrition and Sport, La Trobe University, Melbourne, Australia
}

$\boldsymbol{R}$ ackground and aims: Communication and patient safety in hospitals are B closely related: up to $75 \%$ of adverse hospital events arise from communication failures. Patients with communication difficulties are particularly vulnerable within our health services and are three times more likely to experience preventable safety incidents. Many healthcare professionals have limited access to training and resources to ensure the delivery of safe and effective care to people with communication disability, such as post-stroke aphasia. This study explored the views of the multidisciplinary stroke team regarding care of people with aphasia (PWA).

Method: Purposeful sampling was used to recruit healthcare professionals across disciplines. Focus group data were analysed using qualitative methods. Results: Sixteen healthcare professionals were recruited to an acute $(n=$ $10)$ or rehabilitation focus group $(n=6)$. Exposure to PWA was high; most participants $(n=10)$ encountered PWA several times a week. Yet only two participants felt "very confident" in their knowledge and skills relating to aphasia. Participants noted that interactions with PWA take longer; whilst they understood the need to give more time to PWA, this was viewed negatively for time management. Moreover, healthcare professionals felt challenged in these conversations, unsure of when to use specific strategies. Successful conversations with PWA were associated with increased job satisfaction and improved patient care.

Conclusions: Despite frequent contact with PWA, the multidisciplinary stroke team feel challenged by aphasia. Further research is needed to investigate how to support the delivery of safe and effective multidisciplinary care to PWA.

Correspondence: Marcella Carragher; m.carragher@latrobe.edu.au

\section{From Theory to Practice: Using Autobiographical Memory to Bolster Language Treatment for Early Stage Semantic Dementia}

\author{
Connie Sohn Chaird, ${ }^{1}$ Sajlia Binte Jaliil ${ }^{1,2}$ and Susan Jane Rickard Liow ${ }^{1}$ \\ ${ }^{1}$ Division of Graduate Medical Studies, National University of Singapore \\ ${ }^{2}$ Department of Rehabilitative Services, Changi General Hospital, Singapore
}

$\boldsymbol{R}$ ackground and aims: Word finding difficulties (WFD) are a clinical

$B$ feature of semantic dementia (SD) - a type of primary progressive aphasia. Naming treatment for early stage SD is known to improve picture naming of treated words, however generalisation of such treatment is understood to be poor. It was hypothesised that autobiographical memory could enhance relearning of words in SD. The aim of this study was to see if naming treatment 
bolstered by autobiographical discourse would result in gains beyond picture naming of treated words.

Method: A 61-year-old female participant with early stage SD and mild WFD received language treatment via Skype, twice a week over 4 weeks. 60 functional words were treated with picture naming followed by oral discourse. Homework, consisting of written discourse, was submitted twice a week during treatment period and over a 4-week maintenance period.

Results: Treatment benefits included improved naming of untreated words, reduced WFD in oral discourse, improved self-awareness of disability and improved confidence in overall communication. Significant treatment gains were maintained with homework.

Conclusions: This case highlights the potential for language treatment recruiting autobiographical memory to generalise beyond word level gains. It also shows the benefit of early treatment for patients with this progressive disease.

Correspondence: Connie Chaird; connie.chaird@u.nus.edu

\title{
Acceptability of Cognitive, Functioning, Health and Wellbeing Assessment Tools for Aboriginal and Torres Strait Islander People with Traumatic Brain Injury in Northern Australia
}

\author{
Michelle Fitts, ${ }^{1}$ Taeha Condon ${ }^{2}$ and India Bohanna ${ }^{2}$ \\ ${ }^{1}$ College of Public Health, Medical \& Veterinary Sciences, Australian Institute of Tropical Health \& \\ Medicine, James Cook University, Townsville, Qld, 4810, Australia \\ ${ }^{2}$ College of Public Health, Medical \& Veterinary Sciences, Australian Institute of Tropical Health \& \\ Medicine, James Cook University, PO Box 6811, Cairns, Qld, 4870, Australia
}

$\boldsymbol{R}$ ackground and aims: At present there are no validated instruments for 3 Indigenous traumatic brain injury (TBI) populations. Evaluating the acceptability of the assessment tools for use in a study investigating the transition period for Indigenous Australians following a TBI was the study aim. Tools assessed included the Sentinel Events Questionnaire (SEQ), Strong Living Scale (SLS), Sydney Psychosocial Reintegration Scale (SPRS), Patient Health Questionnaire-9 (PHQ-9 modified), Kessler-10 (K-10), General Anxiety Disorder (GAD) and CogState.

Method: Surveys were conducted with an Indigenous community sample $(n=79)$ upon completion of the tools, seeking feedback about their experiences. Focus groups with Indigenous health workers $(n=8)$ and interviews with hospital staff $(n=9)$ in Northern Queensland and Darwin were also conducted to capture information about their experiences as tool administrators.

Results: Over $90 \%$ of community members considered the questions and response scales on the SLS and mental health screening tools (GAD, PHQ9(modified), K-10) easy to understand. While over $80 \%$ found CogState enjoyable and very easy to understand, $18 \%$ described the experience as being moderately or very stressful. PHQ-9(adapted) was selected over the modified version, as health workers both utilised and preferred this version. K-10 and SPRS were excluded in preference of the GAD and adapted PHQ-9, and SLS. Two themes emerged: confounding of wording by disadvantage and context; the importance of culturally appropriate language and measurement.

Conclusions: Wording modification in response to feedback should assist with appropriateness of the tools. This is the first step towards the development of valid instruments for assessment with this population.

Correspondence: Michelle Fitts; michelle.fitts1@jcu.edu.au 


\title{
The Efficacy and Safety of Extended-Release Methylphenidate Following Traumatic Brain Injury: A Randomised Controlled Pilot Study
}

\author{
Alicia Dymowski, ${ }^{1,2}$ Jennie Ponsford, ${ }^{1,2}$ Jacqueline Owens, ${ }^{1,2}$ John Olver, ${ }^{3,4}$ Michael \\ Ponsford $^{4}$ and Catherine Willmott ${ }^{1,2}$ \\ ${ }^{1}$ Monash Institute of Cognitive and Clinical Neurosciences and School of Psychological Sciences, \\ Monash University, Melbourne, Australia \\ ${ }^{2}$ Monash-Epworth Rehabilitation Research Centre, Epworth HealthCare, Melbourne, Australia \\ ${ }^{3}$ Epworth-Monash Rehabilitation Medicine Unit, Epworth HealthCare, Melbourne, Australia \\ ${ }^{4}$ Epworth Rehabilitation, Epworth HealthCare, Melbourne, Australia
}

\begin{abstract}
$B$ ackground and objectives: Deficits in processing speed and complex attention are common following traumatic brain injury (TBI). This study aimed to investigate the feasibility, safety and efficacy of extended-release methylphenidate in enhancing processing speed, complex attentional functioning and everyday attentional behaviour after TBI.

Method: Eleven individuals with attention and/or processing speed deficits following complicated mild to severe TBI (mean post-traumatic amnesia duration $=63.80$ days, $S D=45.15$ ) participated in a 7-week randomised, placebo-controlled, double-blind parallel pilot study. Participants were allocated according to a blocked randomisation schedule to receive either daily extended-release methylphenidate (Ritalin ${ }^{\circledR}$ LA at a dose of $0.6 \mathrm{mg} / \mathrm{kg}$ ) or placebo (lactose). Measures of processing speed, complex attention and ratings of everyday attentional behaviour were completed at baseline, week 7 (on-drug), week 8 (off-drug) and 9 month follow-up. Vital signs and side effects were monitored from baseline to week 8 .

Results: Three per cent $(n=11)$ of TBI patients screened across a 2.5 year period participated. Results were analysed for six and four individuals on methylphenidate and placebo, respectively. Groups did not differ on attentional or processing speed measures or on relative/therapist ratings of everyday attentional behaviour. One methylphenidate participant withdrew due to difficulty sleeping. Methylphenidate was associated with trends towards increased blood pressure and reported anxiety.

Conclusions: Extended-release methylphenidate was not associated with enhanced processing speed, attentional functioning or everyday attentional behaviour. Alternative treatments for attention deficits after TBI should be explored given the limited feasibility and side effects profile of methylphenidate in the TBI population.
\end{abstract}

Correspondence: Alicia Dymowski; Alicia.dymowski@epworth.org.au

\section{Recovery Patterns in Narrative Discourse in the First Year Post TBI}

Elise Elbourn, Leanne Togher, Belinda Kenny and Emma Power

Discipline of Speech Pathology, University of Sydney, Sydney, Australia

$\boldsymbol{B}$ ackground and aims: Little is known about the recovery of narrative discourse in the first year following TBI. The first year post injury typically involves inpatient rehabilitation and community reintegration. To maximise rehabilitation outcomes we need to better understand recovery of discourse skills.

Method: Participants $(n=58)$ with severe TBI completed a narrative-retelling task at 3 monthly intervals in the first year post-injury, as part of a longitudinal study. Narrative discourse was evaluated using Main Concept Analysis (MCA) of the Cinderella story. 
Results: Many of the individuals in this cohort showed some degree of recovery of their discourse skills $(\sim 65 \%)$ however most of these individuals continued to present with a discourse impairment at 12 months. A large proportion of individuals presented with a similar pattern of impairment across the first year $(\sim 30 \%)$ and a very small group of individuals had a pattern of decline in their discourse skills across the first 12 months $(\sim 1 \%)$. Deeper analysis showed that several distinct discourse profiles emerged, including a group of individuals with many absent elements $(\sim 32 \%)$, individuals with highly disordered narratives $(\sim 13 \%)$ and a group with poor scores relative to the volume of verbal output $(\sim 20 \%)$.

Conclusions: In the first year following a TBI, many individuals will show some degree of improvement in discourse skills but are still likely to have a discourse impairment. This has important implications for rehabilitation services such as continuation of services throughout the first year. Complementary analysis for various subgroups is highly indicated.

Correspondence: Elise Elbourn; elise.elbourn@sydney.edu.au

\title{
Are Conventional Testing Methods Continuing to Turn A Blind Eye? The Mobility Assessment Course (MAC) for the Diagnosis of Spatial Neglect
}

\author{
Megan Grech, ${ }^{1}$ Lindy Williams, ${ }^{2}$ Tracey Stuart $^{3}$ and Tobias Loetscher ${ }^{1}$ \\ ${ }^{1}$ Cognitive Ageing and Impairment Neuroscience Laboratory, School of Psychology, Social Work \\ and Social Policy, Magill Campus, University of South Australia, Adelaide, Australia \\ ${ }^{2}$ School of Health Sciences, City East Campus, University of South Australia, Adelaide, Australia \\ ${ }^{3}$ Guide Dogs of SA/NT, Adelaide, Australia
}

$\boldsymbol{R}$ ackground and aims: Spatial Neglect is a common consequence of stroke resulting in contralesional awareness deficits. The endurance of neglect is a poor predictive indicator for functional autonomy. Therefore, diagnosing neglect promptly and accurately is crucial. The assessment of neglect currently relies on conventional paper-and-pencil tasks. However, the sensitivity and accuracy of these tasks are widely criticised, specifically for their lack of ecological validity. To address this problem, other measures such as the Mobility Assessment Course (MAC) are being employed. Surprisingly, there is no scientific evidence to suggest the MAC is an accurate alternative to conventional tasks.

Method: This study involved the analysis of retrospective data collected by Guide Dogs SA/NT. Performances on paper-and-pencil tasks and the MAC were analysed in a sample of 60 left hemisphere damage (LHD) and 84 right hemisphere damage (RHD) participants. Further, 50 healthy aged-matched controls provided normative data on the MAC.

Results: Contrary to expectations, the MAC was no better at detecting neglect than paper-and-pencil tasks. A sensitivity analysis revealed the MAC as a highly sensitive measure. However, the Receiver Operating Characteristic (ROC) curve provided a more thorough analysis of sensitivity and specificity, revealing poor specificity and a large number of false positives compared to paper-and-pencil tasks.

Conclusions: The MAC is a promising measure. However, in its current form, it is not more diagnostically accurate than conventional tasks. These findings highlight the importance of using sensitivity and specificity analyses when considering new measures for the assessment of neglect.

Correspondence: Megan Grech; mcdmy003@mymail.unisa.edu.au 


\title{
The Information Processing Time in the Brain until the Motor Execution Period can be Evaluated by Analyzing the Whole-Body Reaction Time by the High-Speed Camera Image
}

\author{
Satoru Kai and Koji Nagino \\ Division of Physical Therapy, Faculty of Allied Health Sciences, Kansai University of Welfare Sciences, \\ Osaka, Japan
}

$\boldsymbol{R}$ ackground and aims: In order to execute the motion, it is necessary to process the information processing in the brain, subsequence to send a command to the peripheral skeletal muscle, and muscle contraction. We demonstrate that the jumping motion can be evaluated by dividing it into the motor resting period and the motor execution period.

Methods: The subjects were 16 healthy young people. The subjects make a jumping motion with light as a signal. We measured the shooting of images with a high-speed camera (sampling frequency 1,000 Hz) and the time when both feet took off.

Results: The jumping reaction time is $294 \pm 44 \mathrm{msec}$ on average, the motor resting period (time from start of light cue to move out) is $179 \pm 34 \mathrm{msec}$, and the motor execution period (time from move to the took off of both feet) is $115 \pm 25 \mathrm{msec}$.

Conclusions: So far, nerve conduction velocity is almost constant, motor execution ability related to the motor reaction time. However, by analysing the image at the sampling frequency of $1,000 \mathrm{~Hz}$, we found that the time course of the information processing process in the brain, which is the motor resting period, can be evaluated.

Correspondence: Satoru Kai; kai@tamateyama.ac.jp

\section{Intracranial Pressure Measured by Lumbar Tap in the Patients with Chronic Post-Traumatic Headache}

Kazuyoshi Kato, ${ }^{1}$ Kiyoshi Takagi ${ }^{2}$ and Kenji Onouchi ${ }^{2}$

${ }^{1}$ Department of Surgery, Abiko Seijinkai Hospital

${ }^{2}$ Normal Pressure Hydrocephalus Center, Kashiwa Tanaka Hospital

$\boldsymbol{B}$ ackground and aims: Some patients with chronic post-traumatic 3 headache (CPTH) shows headache with orthostatic nature similar to spontaneous intracranial hypotension (SIH). However, intracranial pressure (ICP) in the patients with CPTH has not yet been known. We investigated the ICP in the CPTH patients with headache of orthostatic nature.

Methods: Four hundred and forty-one CPTH patients with headache of orthostatic nature were admitted and measured ICP by lumbar puncture from July 2004 to January 2017. Results were shown in mean $(S D)$. Statistical analysis performed by Student's t-test and significant level was set $p<.05$.

Results: Mean age was 38.7 (13.8) yo (male: $37.7(13.5)$ yo $(n=187)$, female: $39.5(14.1)$ yo $(n=254))$. There was no significant difference $(p=.175)$. Mean ICP was 146.1 (14.1) $\mathrm{mmH}_{2} \mathrm{O}$ (male: 158.0 (46.2) $\mathrm{mmH}_{2} \mathrm{O}(n=187)$, female: $\left.137.3(40.9) \mathrm{mmH}_{2} \mathrm{O}(n=254)\right)$. Significant difference was noted in ICP between male and female $(p<.0001)$. Only 10 cases showed low ICP less than $60 \mathrm{mmH}_{2} \mathrm{O}$ and 58 cases showed high ICP over $200 \mathrm{mmH}_{2} \mathrm{O}$.

Conclusions: Although the patients complained headache of orthostatic nature similar to SIH, ICP measured by lumbar puncture was not low in most cases. Unexpectedly, female CPTH patents showed significantly lower ICP than the 
male patients. This study indicates the importance of ICP measurement in CPTH.

Correspondence: Kiyoshi Takagi; paulktkg@mac.com

\title{
Cognitive-Communication Screener for Early Identification of Vascular Cognitive Impairment after Acquired Brain Injury
}

\author{
Alena Lixian Low, ${ }^{1,2}$ Sajlia Jalil ${ }^{1,2}$ and Susan Jane Rickard Liow ${ }^{1}$ \\ ${ }^{1}$ Department of Graduate Medical Studies, National University of Singapore \\ ${ }^{2}$ Department of Rehabilitative Services, Changi General Hospital
}

\begin{abstract}
$\boldsymbol{B}$ ackground and aims: Patients with vascular cognitive impairment no dementia (VCIND) post-stroke are at risk of conversion to dementia. Early detection of VCIND would facilitate appropriate intervention, supporting positive outcomes. A cognitive-communication screener was developed in the English language to assess a range of skills such as semantic memory, working memory, verbal memory, attention, visuomotor ability, visuospatial ability, language and executive function.

Method: The screener was normed on 60 neurologically-intact participants: 30 aged $40-59$ years ( 18 females, 12 males), 30 aged 60-78 years ( 11 females, 19 males). The screener was then administered to 10 English-speaking adults with stroke at three time points - first, third and sixth month post-stroke to measure their cognitive-communication skills as well as to track changes over time. The Montreal Cognitive Assessment (MoCA) was also administered as a form of preliminary test of concurrent validity of the screener.

Results: Normative data for $5^{\text {th }}, 10^{\text {th }}, 25^{\text {th }}, 50^{\text {th }}, 75^{\text {th }}, 90^{\text {th }}$ and $95^{\text {th }}$ centile were calculated for each of the age groups. Significant difference in performance between the two age groups point to the need for separate normative data for different age groups, providing a more accurate reference for clinicians. Changes to patients' performance over the three time points on the screener were similar to that of the MoCA.

Conclusion: The cognitive-communication screener appears to be a valid and reliable tool in detecting cognitive-communication deficits and hence a need to further assess for VCIND in patients post-stroke.
\end{abstract}

Correspondence: Alena Low; low.alena@gmail.com

\section{Influence of Galvanic Vestibular Stimulation to Blood Pressure and Autonomic Nerve Activity}

Koji Nagino and Satoru Kai

Division of Physical Therapy, Faculty of Allied Health Sciences, Kansai University of Welfare Sciences, Osaka, Japan

$\boldsymbol{B}$ ackground and aims: Galvanic vestibular stimulation (GVS) has been used for rehabilitation of unilateral spatial neglect in recent years. However, there is no report verifying the effect of GVS used in rehabilitation on blood pressure and autonomic nerve activity. Thus, this study observed blood pressure fluctuation and autonomic nerve activity when GVS was used to healthy men.

Methods: Fourteen healthy men participated in this study. Subsensory GVS was applied over the mastoids (left-anodal, right-cathodal, mean intensity: 0.7 $\mathrm{mA}$ ). Blood pressure and electrocardiogram (ECG) were observed during GVS 
or Sham GVS. Blood pressure was measured continuously at fingertip. Power spectra obtained from spectral analysis were defined as low frequency (LF) and high frequency (HF). LF power shows parasympathetic and LF power to $\mathrm{HF}$ power $(\mathrm{LF} / \mathrm{HF})$ shows sympathetic predominance. A repeated-measure two-way ANOVA assessing group and time was performed.

Results: There was no significant change in blood pressure and autonomic nerve activity even GVS and Sham GVS [Interaction: systolic blood pressure: $F=0.298, p=.995$; diastolic blood pressure: $F=0.459, p=.958$; mean blood pressure: $F=0.401, p=.978$; Heart rate: $F=0.755, p=.727$; HF; $F$ $=0.581, p=.889$ : for $\mathrm{LF} / \mathrm{HF} ; F=0.699, p=.784]$.

Conclusions: These results imply that subsensory GVS is not influence blood pressure and autonomic nerve activity.

Correspondence: Koji Nagino; nagino1213@gmail.com

\section{The Functional Connectivity between the Ventral Tegmental Area and Default Mode Network and Associated Attentional Deficits Following Traumatic Brain Injury}

Jacqueline Owens, ${ }^{1,2,3}$ Orwa Dandash,, ${ }^{4,5}$ Alex Fornito, ${ }^{4,5}$ Jennie Ponsford, ${ }^{1,2,3}$ Gershon Spitz ${ }^{1,2,3}$ and Catherine Willmott ${ }^{1,2,3}$

${ }^{1}$ School of Psychological Sciences, Monash University, Melbourne, Australia

${ }^{2}$ Monash-Epworth Rehabilitation Research Centre, Epworth HealthCare, Melbourne, Australia

${ }^{3}$ Monash Institute of Cognitive and Clinical Neurosciences, Monash University, Melbourne, Australia

${ }^{4}$ Brain \& Mental Health Laboratory, School of Psychological Sciences \& Monash Institute of Cognitive and Clinical Neurosciences, Monash University, Australia

${ }^{5}$ Melbourne Neuropsychiatry Centre, Department of Psychiatry, The University of Melbourne and Melbourne Health, Melbourne, Australia

$\boldsymbol{R}$ ackground and objectives: Dopamine (DA) agonists have demonstrated preliminary efficacy in the amelioration of attentional deficits in individuals with traumatic brain injury (TBI). Elucidating the underlying disruption to dopaminergic pathways is important to inform the rationale for such treatments. This study aimed to explore functional connectivity between the ventral tegmental area (VTA) (a region rich in DA cell bodies) and the default mode network (DMN) following TBI using resting-state functional magnetic imaging (rs-fMRI); and determine whether functional alterations following TBI were associated with attentional performance in the TBI group.

Method: Twenty participants with complicated mild to severe TBI and 20 controls underwent neuropsychological assessment of attentional abilities and rs-fMRI analysis. A seed was placed in the VTA and the resting state analysis was conducted within a mask of the DMN.

Results: Reduced information processing speed was the main finding in the TBI group, when compared to controls. In controls, the VTA demonstrated functional connectivity with the angular gyrus and precuneus. No significant functional connectivity between the VTA and DMN was observed in the TBI group. Between-group differences were, however, not found to be statistically significant.

Conclusion: The individual variability in damage to the neural networks connecting the VTA and DMN caused by TBI may have underpinned the nonsignificant findings in this relatively small TBI sample. No correlations analysis with attentional performance was run given the lack of between-group differences, however, it is unlikely the non-significant differences between the groups would have accounted for the attention deficits observed in this sample.

Correspondence: Jacqueline Owens; jacqueline.owens@monash.edu 


\title{
High Levels of Brain Impairment in Methamphetamine-Dependent Individuals: Impact on Memory, Attention and Decision-Making
}

\author{
Adam Rubenis, ${ }^{1}$ Rebecca Fitzpatrick, ${ }^{1}$ Dan Lubman ${ }^{1,2}$ and Antonio Verdejo- \\ Garcia $^{1}$ \\ ${ }^{1}$ School of Psychological Sciences, Monash University, Melbourne, Australia \\ ${ }^{2}$ Turning Point, Eastern Health Melbourne, Australia
}

\begin{abstract}
$\boldsymbol{B}$ ackground and objectives: Methamphetamine-related harms are on the B rise in Australia, including increased ambulance attendances and higher dependence rates. Methamphetamine-dependent individuals exhibit poorer cognitive performance compared to non-users. However, it is debated if these performance differences reflect clinically significant cognitive impairments, and in which domains.

Method: We conducted an observational cross-sectional study of 108 participants with methamphetamine dependence, recruited from rehabilitation/detoxification facilities and the community. Participants were screened for medical/mental health conditions that impact cognition. Participants were tested after 2-21 days of abstinence via a cognitive battery that measured attention (Continuous Performance Test-2), memory (Hopkins Verbal Learning Test-Revised) and decision-making (Iowa Gambling Task). Results were compared to standardised population norms to determine clinically significant impairment, defined as $\geq 1.5$ standard deviations below the corresponding mean.

Results: In the Hopkins verbal memory test, $28 \%$ of participants had clinically significant impairment in recall memory and $40 \%$ in recognition memory. In the Continuous Performance Test, $35 \%$ of participants had clinically significant impairment in the impulsivity index, $29 \%$ in the perseveration index, and $19 \%$ in the inattention index. In the Iowa Gambling Task of decision-making, $17 \%$ of participants had clinically significant impairment in the 'A' index, measuring preference for decisions with high/frequent rewards and high/frequent punishments.

Conclusions: A high proportion of methamphetamine users show clinically significant impairment in measures of memory, impulsivity, perseveration and decision-making. Findings suggest significant problems in retrieving information relevant to their drug use (e.g. history of reward/punishment) and selfcontrol. Treatment may be improved with a tailored approach incorporating cognitive remediation.
\end{abstract}

Correspondence: Adam Rubenis; adam.rubenis@monash.edu

\section{Speech Pathologists Practice in Post Stroke Aphasia Rehabilitation: Gaps in Training and Competency in Counselling}

\author{
Jasvinder Sekhon, ${ }^{1}$ Jennifer Oates, ${ }^{1}$ Ian Kneebone ${ }^{2}$ and Miranda Rose ${ }^{1}$ \\ ${ }^{1}$ School of Allied Health, La Trobe University, Melbourne, Australia \\ ${ }^{2}$ Discipline of Clinical Psychology, Graduate School of Health, University of Technology Sydney, \\ Sydney, Australia
}

ackground and aims: Speech pathologists (SPs) report utilising a range
of approaches including counselling, to facilitate psychological wellbe-
ing in people impacted by post stroke aphasia. However, SPs report feeling
under skilled in counselling and attribute this to a lack of training. A variety
of approaches to manage psychological wellbeing after stroke are described.
However, many of these studies exclude people with significant aphasia. We 
aimed to 1) summarise the counselling approaches and elements used to facilitate psychological wellbeing in people with post stroke aphasia; 2) summarise current SP counselling training; and 3) Identify any gaps in counselling training.

Method: A systematic literature review of SP counselling training was undertaken. Results were compared to counselling approaches used to facilitate psychological wellbeing in stroke survivors with aphasia. Common counselling behaviours, terms, and theories were identified and synthesised.

Results: SP counselling training is generic and limited. SPs receive little training in counselling to address the psychological wellbeing of people with aphasia and their families. A wide range of approaches with potential to improve psychological wellbeing in stroke survivors with aphasia were identified.

Conclusions: SPs are trained to work with people with aphasia and are key health professionals in stroke rehabilitation. People with aphasia may miss out on essential psychological care provided by non-SPs due to barriers with communication access. SPs require appropriate training in counselling to provide effective and timely low level psychological care to people impacted by post stroke aphasia in order to facilitate optimal rehabilitation gains.

Correspondence: Miranda Rose; M.Rose@latrobe.edu.au

\title{
The Structure of the Rivermead Post Concussion Symptoms Questionnaire in Australian Adults with Traumatic Brain Injury
}

\author{
Matt Thomas, ${ }^{1,3}$ Clive Skilbeck ${ }^{2,3}$ and Mark Slatyer ${ }^{3}$ \\ ${ }^{1}$ School of Psychology, Charles Sturt University, Bathurst, NSW, Australia \\ ${ }^{2}$ School of Psychology, University of Tasmania, Hobart, TAS, Australia \\ ${ }^{3}$ Neurotrama Register of Tasmania, Royal Hobart Hospital, TAS, Australia
}

$\boldsymbol{R}$ ackground and aims: Many sustaining traumatic brain injury suffer on3 going post-concussion symptoms (PCS). The Rivermead Post Concussion Symptoms Questionnaire (RPQ) is one widely utilised measure of PCS. However, there has been disagreement about its structure. This study compared the fit of published structures of the RPQ with a four-factor structure derived from a large sample of adults with TBI in Tasmania.

Method: A sample of 661 adults with TBI completed the RPQ approximately 1 month after injury. An Exploratory Factor Analysis (EFA) using the first half of the sample $(n=330)$ suggested a four-factor solution. This structure was then compared with 1,2 and 3-factor models reported in the literature within the second half of the sample $(n=331)$. Trajectory of recovery across these factors was examined within this sample at time-points to 12 months following injury.

Results: RPQ items were severely positively skewed. Spearman's correlations showed some stronger item grouping for aspects of somatic, cognitive and emotional functioning. Results of the EFA identified a four-factor model. The comparative fit of the models was examined utilising bootstrapping. The data best fitted the four-factor model, which also had adequate internal consistency and appeared clinically useful, as there were some differences in recovery across these constructs up to 12 months following injury.

Conclusions: The data best fitted a four-factor model for RPQ items: Mood and somatic, Cognitive, Vertigo and Vision items. This structure was identified using a rigorous statistical approach, and appeared clinically useful. Future research could attempt replication within international samples.

Correspondence: Matt Thomas; mathomas@csu.edu.au 


\title{
Using a Multiple-Component Strategy-Based Intervention for Reading Comprehension Deficits after ABI
}

\author{
Kerrin Watter, ${ }^{1,2}$ Anna Copley $^{2}$ and Emma Finch ${ }^{1,2,3}$ \\ ${ }^{1}$ Speech Pathology Department, Princess Alexandra Hospital, Brisbane, Australia \\ ${ }^{2}$ School of Health and Rehabilitation Science, University of Queensland, Brisbane, Australia \\ ${ }^{3}$ Centre for Function and Health Research, Metro South Health, Brisbane, Australia
}

\begin{abstract}
$\boldsymbol{B}$ ackground and aims: Cognitive-communication reading comprehension B (CCRC) deficits can impact the activity and participation of people with acquired brain injury (pwABI). Targeting CCRC deficits in early rehabilitation may improve rehabilitation participation and independence for more complex reading and self-management tasks on discharge. This project aimed to develop and trial a multiple-strategy CCRC intervention, involving visual, content and metacognitive strategies.
\end{abstract}

Method: The intervention was developed from clinical and research based evidence (levels I-V), using an emergent multi-phase mixed methodology. The intervention was conducted with an adult with ABI during subacute rehabilitation, using an experimental single case study design with multiple baselines (MB-SCED). Pre-, post- and follow-up formal assessment of CCRC skills occurred via an independent assessor.

Results: Improved CCRC skills were demonstrated via MB-SCED probe results, with a significant change ( $>2 S D$ ) in functional reading, and improvements $(>1 S D)$ in factual and inferential reading. Formal assessment showed improved reading comprehension pre-/post-intervention ( $>2$ years) and at follow up (post/follow-up: 3 years). Strategy use was observed post-assessment but not follow-up.

Conclusions: Multiple-strategy interventions for CCRC deficits were successful in improving and maintaining reading for a pwABI during subacute rehabilitation. Further investigation of the intervention with additional pwABI is warranted.

Correspondence: Kerrin Watter; Kerrin.Watter@health.qld.gov.au 